\title{
Measurements of Fundamental Fluid Physics of SNF Storage Canisters
}

K. G. Condie

G. E. McCreery

D. M. McEligot

September 2001

Idaho National Engineering and Environmental Laboratory Bechtel BWXT Idaho, LLC 
INEEL/EXT-01-01269

\title{
Measurements of Fundamental Fluid Physics of SNF Storage Canisters
}

\author{
Keith G. Condie \\ Glenn E. McCreery \\ Donald M. McEligot
}

September 2001

Idaho National Engineering and Environmental Laboratory Idaho Falls, Idaho 83415

Prepared for the U.S. Department of Energy

Assistant Secretary for Environmental Management

Under DOE Idaho Operations Office

Contract DE-AC07-99ID13727 


\title{
Measurements of fundamental fluid physics of SNF storage canisters
}

\author{
Keith G. Condie, Glenn E. McCreery and Donald M. McEligot
}

\begin{abstract}
Summary
With the University of Idaho, Ohio State University and Clarksean Associates, this research program has the long-term goal to develop reliable predictive techniques for the energy, mass and momentum transfer plus chemical reactions in drying / passivation (surface oxidation) operations in the transfer and storage of spent nuclear fuel (SNF) from wet to dry storage. Such techniques are needed to assist in design of future transfer and storage systems, prediction of the performance of existing and proposed systems and safety (re)evaluation of systems as necessary at later dates.
\end{abstract}

Many fuel element geometries and configurations are accommodated in the storage of spent nuclear fuel. Consequently, there is no one generic fuel element / assembly, storage basket or canister and, therefore, no single generic fuel storage configuration. One can, however, identify generic flow phenomena or processes which may be present during drying or passivation in SNF canisters. The objective of the INEEL tasks was to obtain fundamental measurements of these flow processes in appropriate parameter ranges.

With the University of Idaho, an idealization of a combined drying and passivation approach has been defined in order to investigate the generic flow processes. This simulation includes flow phenomena that occur in canisters for high-enrichment and medium-enrichment fuels, where fuel element spacing in the canister is increased as compared with low enrichment fuel. Canister diameter was taken as $46 \mathrm{~cm}$ (18 in.) and a single basket of about 1.3 meters (4 ft.) length was considered. A long central tube ("dip tube") served as the inlet as in one earlier concept for a passivation process; while this concept apparently has not yet been selected for application, it provides an excellent example of the coupled, complex phenomena which may be present in canister flows. Suggested design flow rates for this hypothesized application indicate that the Reynolds number in the inlet tube would be expected to be between 2500 and 5000, i.e., relatively low.

The local distributions of convective mass transfer characteristics (drying/passivation) are expected to depend on the freestream turbulence in the flow around stored fuel elements. The magnitudes of this turbulence depend on the turbulence distributions on the upstream side of the perforated basket support plate ("inlet plenum") and, in turn, in the impinging jet and in the inlet tube.

To date the INEEL efforts have emphasized two tasks:

- Overall flow visualization for a variety of configurations and

- Pointwise turbulence and velocity measurements with two-component laser Doppler velocimetry in the unique INEEL Matched-Index-of-Refraction flow system

This information can assist engineers in understanding variations of surface drying and passivation through an array and approaches to modify designs to counter non-uniformities and to improve 
distribution, as well as providing bases for assessment of computational fluid dynamics codes proposed for the purpose.

A water-flow experiment with a 3/4-scale model (relative to the idealized canister) has been used for overall flow visualization and velocity measurements, with and without an array of simulated fuel elements. Observations have been made with perforated plates (representing basket support plates) having three hole geometries, with and without simulated fuel elements. Streamline and velocity vector plots are presented. The flow patterns exhibited are very complex and provide a non-uniform flow distribution along the simulated fuel elements. Experiments were conducted to determine the canister flow field with three perforated plates with open areas of $50 \%, 8 \%$ and $4 \%$ (porosities). Experiments reported are primarily for $\mathrm{Re}_{\mathrm{inl}} \mathrm{let}=2,500 \pm 100$.

The flow is approximately circumferentially periodic and symmetrical about cross-sections through the four outer tubes for experiments using each of the three perforated plates. With a 50\% open area plate, the flow pattern is comparable to that of a submerged impinging jet on a semiinfinite plate with formation of a single large vortex and entrainment and recirculation through the holes in the perforated plate near the center. With the $4 \%$ and $8 \%$ open area plates, recirculation regions occur downstream of the perforated plate and two main recirculation zones (plus a small corner flow vortex) form upstream of the perforated plate. In these cases the flow pattern in the inlet plenum has some features of a confined impinging jet .

The Reynolds number in the array downstream of the plate based on hydraulic diameter is approximately 70 for $\mathrm{Re}_{\text {inlet }}=2,500$. With the tubes present, vortices persist for the majority of the tube bundle length and the flow only starts to approach a fully-developed laminar profile near the exit of the basket $\left(\mathrm{L} / \mathrm{Dh}_{\mathrm{h}} \approx 6\right)$. The flow distribution is uneven near the surface of the simulated fuel elements. These experiments show flow across the simulated elements and resultant vortex shedding, although the vortices do not show the clear alternating pattern of a Karman vortex street.

Velocity and turbulence distributions were measured with laser Doppler velocimetry in the unique INEEL Matched-Index-of-Refraction (MIR) flow system. An advantage of this facility is the capability of obtaining optical fluid mechanics data without optical distortion and without disturbing the flow by inserting a physical sensor. A 0.6-scale model of the idealized canister was employed with dimensions as follows: plenum spacing $H / D=s / D=2$, canister internal radius $r_{0} / D=9$, pitch of holes in the perforated plate $\mathrm{ph} / \mathrm{D}=1$ and hole diameter $\mathrm{d}_{\mathrm{h}} / \mathrm{D}=1 / 4$ in a square pattern. Most measurements were taken at Reinlet $=2510$. This part of the study concentrated on flow in the inlet tube, the impinging jet and flow upstream of the perforated plate (inlet plenum).

Measurements included the mean distributions of radial and axial velocity components, $V$ and $U$, and their root-mean-squared fluctuations, $v^{\prime}$ and $u^{\prime}$. Main features of the flow in the inlet plenum upstream of the perforated plate are the high velocity impinging jet, the thin expanding wall jet, a single recirculating eddy near the outer wall of the canister and relatively low velocities near the perforated plate. The flow in the wall jet remains attached until it impinges on the outer wall of the plenum region and induces the main recirculating eddy. In the plenum region upstream of the perforated plate, the radial velocity component at a point is generally greater than the axial component, except in the recirculating eddy. The rms radial velocity fluctuations are generally considerably larger than the rms axial fluctuations. The large relative values of $\mathrm{v}^{\prime}$ imply the 
likelihood that there are considerable fluctuations in the incident angle as the flow approaches the holes in the perforated plate; this situation differs significantly from flow conditioning applications of perforated plates in wind tunnels.

It is apparent from these experiments that, despite the conceptual simplicity of the canister design, the flow pattern is very complex. Comparison of the present results shows that flow in the plenum region upstream of the perforated basket support plate is sensitive to the inlet flow characteristics in the expected range of Reynolds numbers. What has not been determined is whether turbulent, laminar or transitional inlet flow is preferable for desired drying characteristics; further measurements are required to answer this question. Some additional "lessons learned" are included in the section on concluding remarks.

It is recommended that the present data be used to assess capabilities of existing and proposed CFD (computational fluid dynamics) codes which are intended to predict behavior in SNF canisters. While proprietary codes for predicting drying supposedly have been validated for the nuclear reactor industry, it is not clear that these "validations" have been at appropriate Reynolds numbers and geometrical ranges or how close the predictions were to any measurements. It would be desirable to test such codes by comparison to the present measurements.

The emphases of further recommended work should concentrate on experiments examining

- the effects of a sparsely perforated plate on the turbulence field, downstream and upstream - decay of turbulence fields in simulated fuel element arrays, both sparse (highly-enriched and moderately-enriched) and dense (low enrichment)

plus related velocity fields. These studies would address the topics from the initial proposal that were deferred due to a 45 percent reduction in funds awarded relative to the originally proposed budget. The existing MIR model for these fluid physics experiments can be used, with and without simulated fuel elements. Also the flow visualization model can be employed for rapid examination of a variety of potential configurations.

Measurements should be obtained by Particle Tracking Velocimetry (PTV) with the OSU Moving Particle Tracking system (MPT) and by laser Doppler velocimetry (LDV). Individual particles may be tracked and the overall flow field may be examined quantitatively via flow visualization deduced from application of the MPT system. To supplement the velocity field data and particle tracks deduced via the MPT/PTV methods, measurement of instantaneous velocity components should continue by LDV at fixed positions, primarily in the array. Additional parameters or phenomena which should be investigated systematically include inlet Reynolds number, inlet geometry, fuel rod loading, hole pattern and length in the basket support plate and other pertinent design quantities.

The results of such studies should provide new fundamental knowledge on

- statistics of particle and passive scalar transport from laGrangian measurements in a rod bundle

- laminarization of turbulent flows entering an array of elements, in combined cross and longitudinal flow 
- turbulence behavior in the surroundings of a semi-confined impinging jet plus the benchmark data for code assessment. 


\section{Measurements of fundamental fluid physics of SNF storage canisters Contents}

Summary

Table of contents

Acknowledgments

Nomenclature

ix

xi

Introduction

Background

Related work

Scientific needs

Engineering needs

$\begin{array}{ll}\text { INEEL studies } & 14\end{array}$

Generic idealization $\quad 14$

$\begin{array}{ll}\text { Objectives } & 16\end{array}$

Choice of experiments $\quad 16$

Overall flow visualization $\quad 17$

INEEL Matched-Index-of-Refraction flow system $\quad 27$

Turbulence and velocity measurements in MIR flow system by laser Doppler velocimetry 33

$\begin{array}{lr}\text { Concluding remarks } & 50\end{array}$

$\begin{array}{ll}\text { Recommendations for further work } & 55\end{array}$

Table I. Measurements of turbulent fluid physics of single-phase, axisymmetric impinging 59 jets59

References cited 


\section{ACKNOWLEDGMENTS}

This study was sponsored by the Environmental Management Science Program (EMSP) of the United States Department of Energy (DoE) under DoE Idaho Field Office Contracts DE-AC0794ID13223 and DE-AC07-99ID13727. 


\section{NOMENCLATURE}

\{\} function of

$\mathrm{A}_{\mathrm{cs}} \quad$ cross-sectional area

D tube diameter; $\mathrm{D}_{\mathrm{O}}$, disc diameter (Table I)

$\mathrm{Dh} \quad$ hydraulic diameter, $4 \mathrm{~A}_{\mathrm{cs}} / \mathrm{P}$

f frequency

g acceleration of gravity

$\mathrm{g}_{\mathrm{c}} \quad$ units conversion factor, e.g., $1 \mathrm{~kg} \mathrm{~m} /\left(\mathrm{N} \mathrm{s}^{2}\right), 32.174(\mathrm{lbm} / \mathrm{lbf}) /\left(\mathrm{ft} / \mathrm{sec}^{2}\right)$, etc.

H spacing between surfaces

$\mathrm{k} \quad$ turbulent kinetic energy

L length

$\dot{\mathrm{m}}$ mass flow rate

P $\quad$ wetted perimeter

$\mathrm{p} \quad$ pressure

$\mathrm{p}_{\mathrm{h}} \quad$ pitch

r radial coordinate; $r_{0}$, canister wall radius

$\mathrm{R} \quad$ canister wall radius

s spacing from jet inlet to impingement plate

T absolute temperature

t thickness

U time-mean axial velocity component

$\mathrm{u}^{\prime} \quad$ rms axial velocity fluctuation 


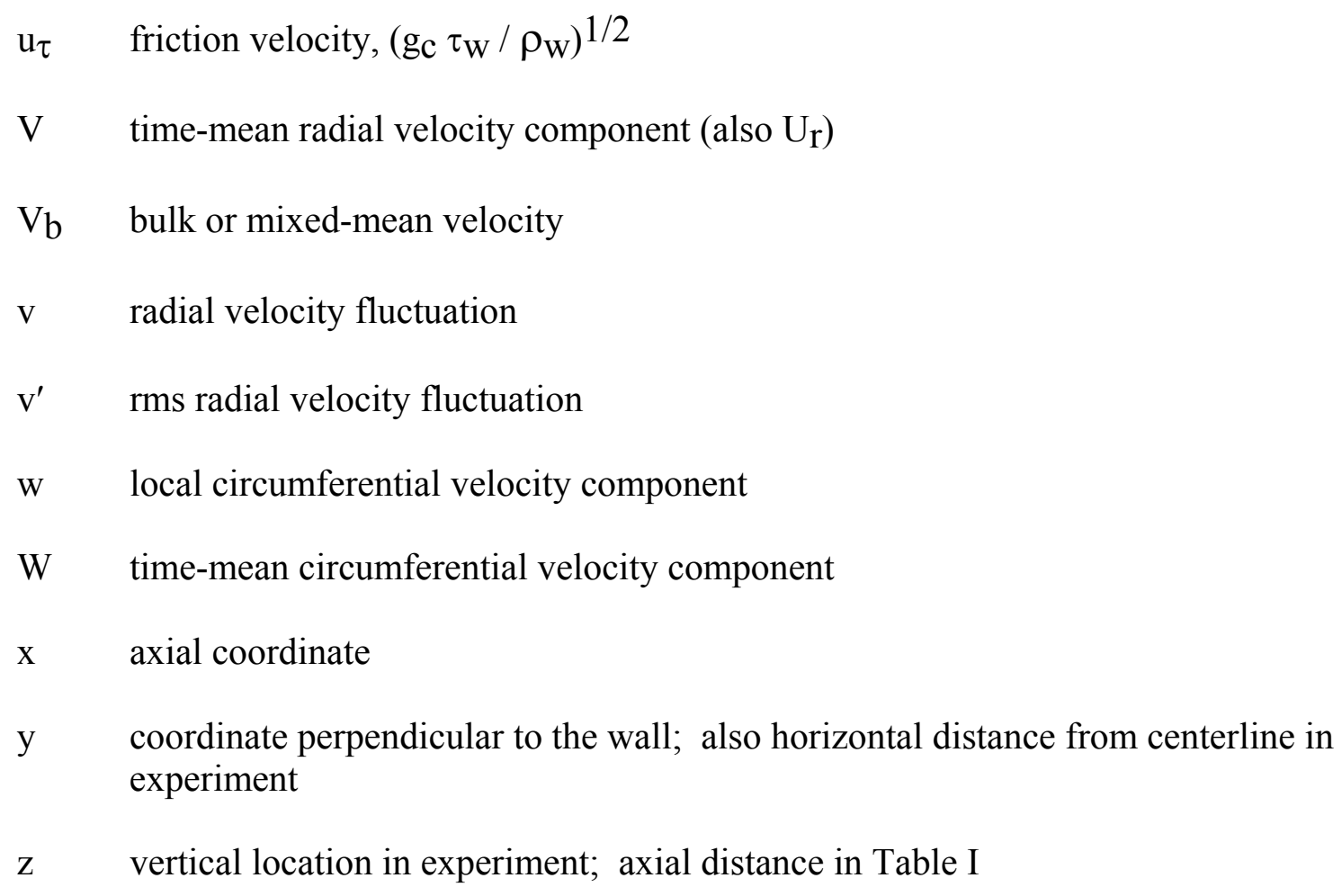

Non-dimensional quantities

$K_{p} \quad$ streamwise pressure gradient, $\left(v /\left(\rho u_{\tau}^{3}\right)\right) g_{c} d p / d x$

$\mathrm{K}_{\mathrm{V}} \quad$ acceleration parameter, $\left(\mathrm{v} / \mathrm{Vb}^{2}\right)\left(\mathrm{dV}_{\mathrm{b}} / \mathrm{dx}\right)$

$\mathrm{L}^{+} \quad$ length, $\mathrm{L} /\left(\mathrm{r}_{\mathrm{W}} \mathrm{Re}\right)$

Re Reynolds number, $4 \dot{\mathrm{m}} / \Pi \mathrm{D} \mu$ for tube; ReD,h, based on hydraulic diameter, $\mathrm{V}_{\mathrm{b}} \mathrm{Dh}_{\mathrm{h}} / \mathrm{v}$

St Strouhal number, e.g., $\mathrm{fD} / \mathrm{U}$

$\mathrm{u}^{+} \quad$ streamwise velocity component, $\mathrm{U} / \mathrm{u}_{\tau}$

$\mathrm{x}^{+} \quad$ axial distance, $\mathrm{x} /\left(\mathrm{r}_{\mathrm{W}} \mathrm{Re}\right)$

$\mathrm{y}^{+} \quad$ wall distance coordinate, $\mathrm{y}\left(\mathrm{g}_{\mathrm{c}} \tau_{\mathrm{W}} / \rho_{\mathrm{W}}\right)^{1 / 2} / v_{\mathrm{W}}$

\section{$\underline{\text { Greek symbols }}$}




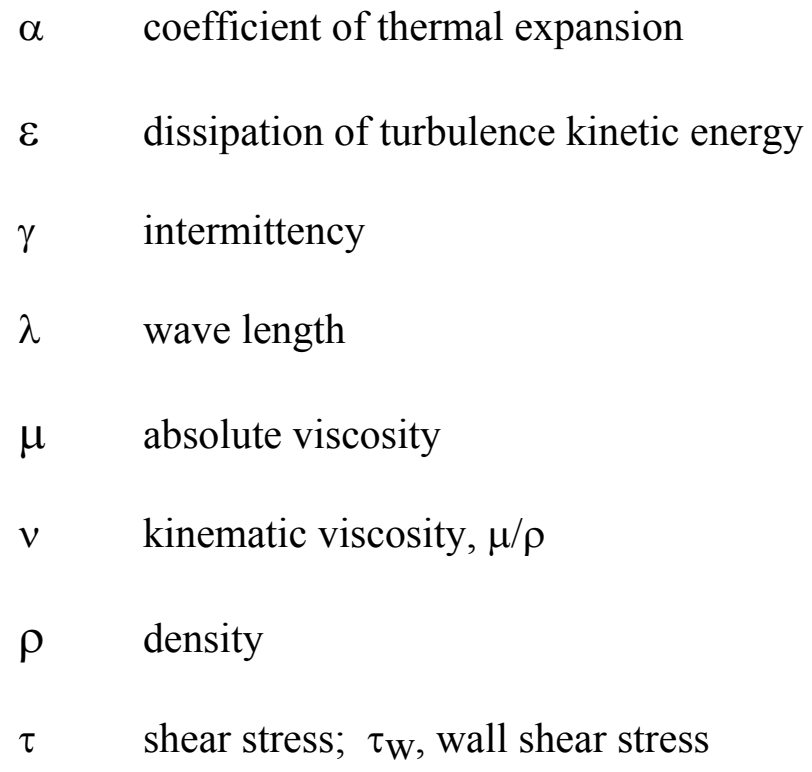

\section{$\underline{\text { Subscripts }}$}

b evaluated at bulk or mixed-mean temperature

c centerline

h hole

in inlet

w wall, evaluated at wall temperature 
xiv 


\section{INTRODUCTION}

\section{Background}

The general (long term) goal of this program is to develop reliable predictive techniques for the energy, mass and momentum transfer plus chemical reactions in drying and passivation operations in the transfer of Spent Nuclear Fuel (SNF) from wet to dry storage. Such techniques are needed to assist in design of transfer and storage systems, prediction of the performance of existing and proposed systems and safety (re)evaluation of systems as necessary at later dates. The goal of the present project is to provide the scientific knowledge required to develop numerical predictive methods treating the key flow phenomena in generic simulations of drying / passivation problems in Environmental Management. The objective of the INEEL tasks is to obtain fundamental measurements of the basic velocity and turbulence fields in generic idealizations of flow processes in SNF canisters during transfer and/or storage; proposed operating conditions give typically lowReynolds-number flows.
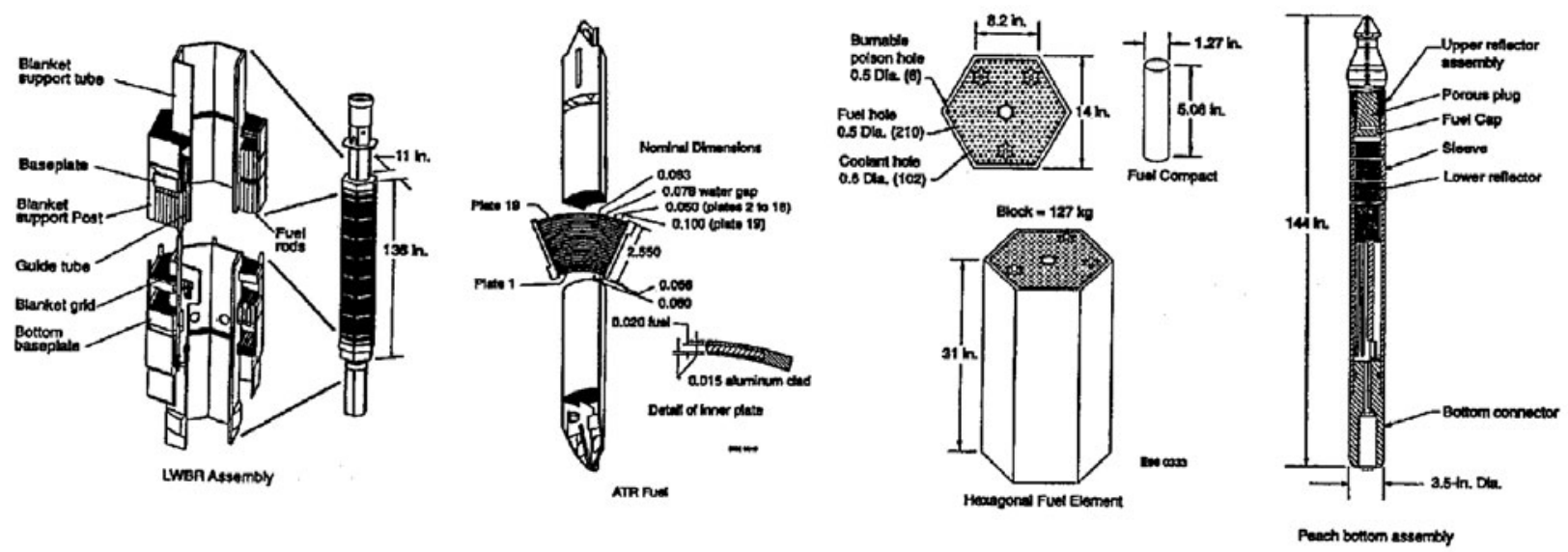

Fig. 1. Examples of fuel elements considered for storage in SNF canisters.

A wide variety of fuel element geometries are accommodated in the storage of spent nuclear fuel. The fuel may have high-, medium- or low-enrichment so criticality considerations affect the number of fuel elements that can be stored in a single fuel basket or canister. Typically, only a few high- or medium-enriched elements can be stored in one basket whereas fuel elements with lowenrichment can be packed densely, approaching the appearance of arrays in a shell-and-tube heat exchanger. Element shapes may be circular rods, annuli, plates, squares, "trapezoids," hexagons, etc. (Figure 1). Proposed canister sizes range from 14 to 24 inches $(36$ to $60 \mathrm{~cm}$ ) with lengths of ten to fourteen feet (3-4 m). Typically, elements or assemblies are placed in baskets for dry storage, 
with drain holes in the basket support plates at the bottom; the hole pattern gives an open-area-ratio of three to nine per cent, depending on the design. The number of baskets vertically in a canister depends on the lengths of the fuel elements being stored. Consequently, there is no one generic fuel element/assembly and, therefore, no single generic fuel storage configuration.

One can, however, identify generic flow phenomena and paths. For example, in a passivation and/or drying system once suggested with flow arranged to pass from one end of a canister to the other (as in one earlier Hanford concept), the sequential flow paths would be

Inlet via downflow in a tube ("dip tube")

Free jet

Impinging jet, spreading radially in a semi-confined region (formed between the bottom of the canister and the bottom support plate of the basket)

Flow through bottom(s) of basket(s) - perforated plate (contraction/expansion, jets)

Vertical flow paths in parallel

Around fuel elements/assemblies

Through fuel element/assembly passages

Bypass flows around baskets, through gaps, etc.

Possible flow into an upper plenum (sudden expansions in parallel)

(With multiple basket layers these last three items will repeat)

Contraction into an exit(s)

The passivation and/or drying of the fuel is accomplished by convective mass transfer from the gas flow through and around the fuel elements. Distribution (or maldistribution) is determined by the relative flow resistances of all the paths. While this concept apparently has not been selected for application, it provides an excellent example of the coupled, complex phenomena which may be present in canister flows. 
For effective passivation of potential pyrophoric reactions, a design aim would be controlled and evenly-distributed, high mass transfer rates to the fuel elements; these rates will depend on the flow pattern and turbulence levels in the vicinity of the elements. As noted in Figure 2, the fluid mechanic design for optimizing this process leads to a sequence of questions concerning the turbulence levels and flow patterns from the reacting surface to the inlet flow. In order to solve these questions several fundamental flow features are involved:

- fluctuating velocity components in low-Reynolds-number turbulent or transitioning flow in a tube

- turbulent transport and possible laminarization of a semi-enclosed impinging jet flow

- low-Reynolds-number flow through an array of holes (basket support plates)

- decay of turbulence in flow around an array of fuel elements

These features can be considered to be the generic flow phenomena of interest in the fluid physics of SNF transfer and storage. Despite extensive literature on impinging jets and flows in arrays, fundamental measurements of these phenomena at the conditions appropriate for some proposed SNF applications are meager to non-existent.

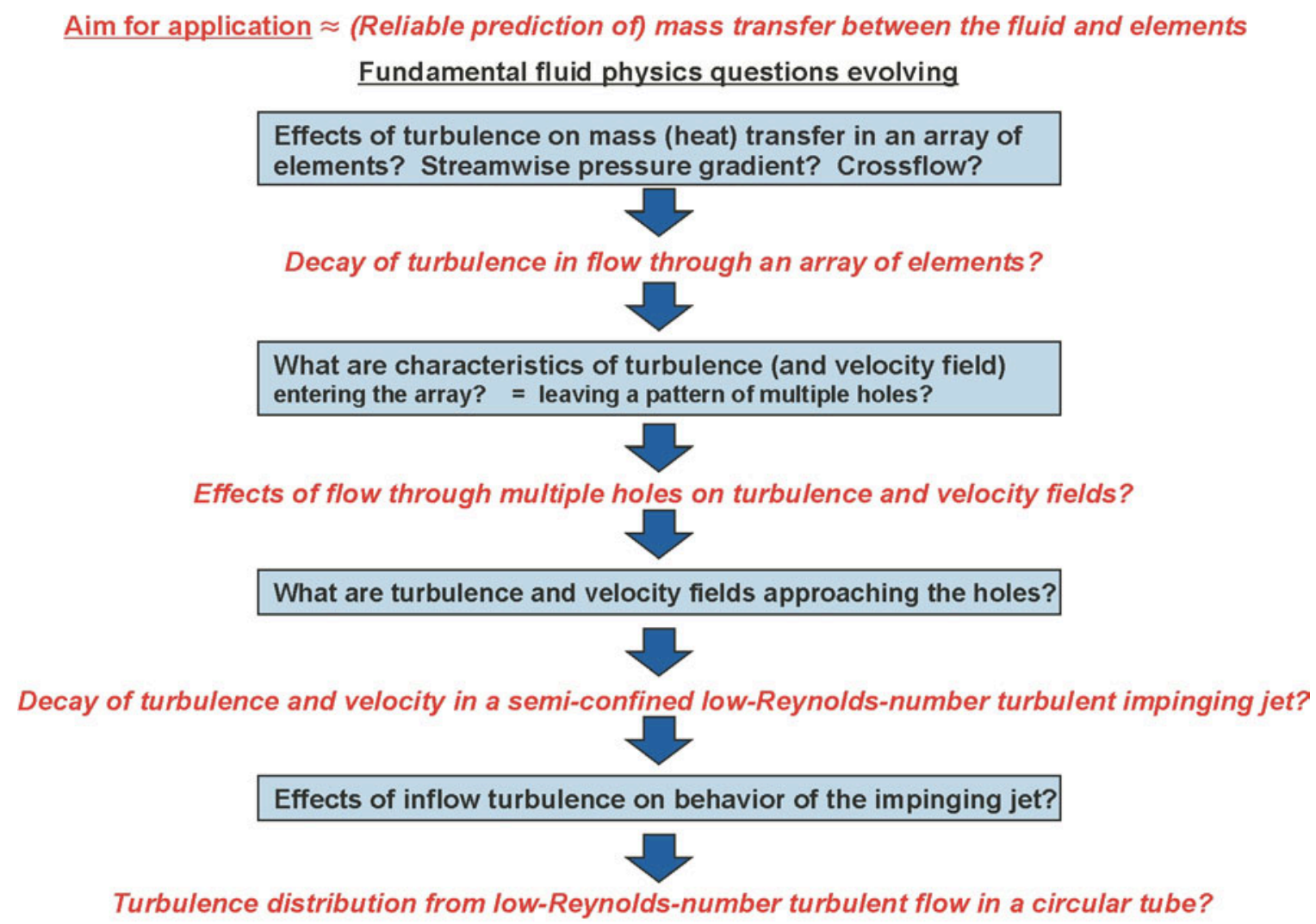

01-GA50936-04

Fig. 2. Identification of fundamental questions and flow phenomena in the fluid physics of SNF canisters. 


\section{Related work}

In addition to flow visualization by dye injection and related methods, the non-invasive optical techniques for flow measurements which are currently popular include laser Doppler velocimetry (LDV), particle image velocimetry (PIV) and particle tracking velocimetry (PTV). All have sufficient maturity as approaches for flow field measurement that systems are available commercially.

McCreery, McKellar and Stoots [1993] at INEEL employed dye-tracking and image analysis techniques to determine velocity vectors in the complicated flow through a quarter-scale, sixthsector model of the moderator tank of a Savannah River Site reactor. Both longitudinal and cross flows occur in this configuration as the flow enters at the bottom from an array of circular cooling tubes, then flows upwards and across them before leaving the tank from an exit also at the bottom. Velocity vectors were determined in the spaces between the rows of tubes; a curved reflecting surface was used to avoid parallax and thereby view several parallel passages simultaneously from a single camera. Velocities were determined from the motions of the dye parcels on sequential frames from the video cameras. In viewing the flow of the dye and displaying the observations, the images of the tubes themselves were electronically subtracted from the data to improve the presentation of the data.

The principles of laser Doppler anemometry have been explained well in a number of reference books (e.g., Durst, Melling and Whitelaw [1976], Albrecht [1986], Adrian [1993] and a couple biennial series of conferences) and use of LDVs has become widespread. The reader is referred to these sources for background and previous work on the topic. Currently over 90 per cent of the new LDV system market uses fiber-optic probes in order to ease handling, in contrast to traditional optical systems. (However, challenges remain [Tropea, 1995].)

Flow visualization and laser Doppler velocimetry were applied by Berner, Durst and McEligot [1984] to approximately two-dimensional flow around a series of segmental baffles in order to simulate important aspects relating to shellside flow in shell-and-tube heat exchangers. With Reynolds numbers from 600 to 10,500, laminar, transitional and turbulent flow were studied; mean and rms velocity profiles were presented for the streamwise-periodic region. The range of measurements was extended by Habib, Durst and McEligot [1984] to include more detailed data on the mean velocity and turbulence distributions for both horizontal and vertical components. 
Particle image velocimetry, a subset of pulsed laser velocimetry, has become popular over the last decade for instantaneous measurement of two- and three-dimensional velocity fields as opposed to the pointwise measurements of an LDV system. For general background, the reviews of Adrian [1986, 1991] are suggested. High density PIV (i.e., using high concentrations of particles) is usually called PIV whereas the low density mode is labeled PTV; in the former, one measures the displacements of small groups of particles whereas tracking of individual particles is feasible with the low-image-density of PTV (hence the title). Each mode has advantages. In "standard" doublepulsed PIV, two images are made of a large number of particles. This technique is useful for making instantaneous maps of flow fields but obtaining time-resolved results for turbulent flows is hampered by the large computational requirements.

For small fields of view, particles may be tracked continuously by an approach such as multiplepulse particle image velocimetry (MP-PIV) provided the particle concentration is small [Ramer and Shaffer, 1992], effectively a version of PTV. The camera is fixed and, in the experiment of Ramer and Shaffer, the width of the pulsating light sheet is limited to about $10 \mathrm{~cm}(\sim 4 \mathrm{in}$.). This approach allows the study of the time evolution of particle trajectories while the particle(s) is in the field of view.

For tracking of individual particles in three-dimensional motion over a large volume, PTV methods provide a better starting point than high-image-density PIV. Worldwide, PTV approaches have been developed by several groups: Guezennec and Brodkey at Ohio State -- our present collaborators [Guezennec et al., 1994], Kasagi at Tokyo [Nishio, Kasagi and Hirata, 1989; Kasagi and Nishio, 1991; Kasagi and Matsunaga, 1995], Rockwell at Lehigh [Robinson and Rockwell, 1993] and others. Kasagi's group has applied the technique to unsteady laminar Couette flow, isotropic decaying turbulence, fully-developed turbulent channel flow, an axisymmetric free jet and other interesting basic flows. Typical volumes examined for three-dimensional PTV have been $4 \mathrm{x} 4$ x $4 \mathrm{~cm}^{3}$ [Nishio and Kasagi, 1989], $10 \times 10 \times 1.5 \mathrm{~cm}^{3}$ [Papantoniou and Dracos, 1990], 8 × 8 × 8 $\mathrm{cm}^{3}$ [Kasagi and Matsunaga, 1995] and $6 \times 6 \times 6 \mathrm{~cm}^{3}$ [Ushijima and Tanaka, 1996], i.e., small compared to the volume treated in the present study. The possibility of tracking individual particles through larger volumes was demonstrated by Lowe et al. [1993]. For near-Poiseuille flow in a curved circular tube, they employed a rotating camera which had a field-of-view that moved interactively along the tube with the particle motion; since the tube was only about $4 \mathrm{~cm}$ in diameter, camera motion in a single direction sufficed.

Profs. Guezennec and Brodkey have been developing and applying state-of-the-art Particle Tracking Velocimetry (PTV) for about a decade [Guezennec et al., 1994]. A 3-D PTV technique has 
been developed to provide time-resolved, three-dimensional velocity field measurements throughout a finite volume. The data acquired form a time sequence of stereo images of flow tracer particles suspended in the fluid. The technique has been optimized to allow fully automatic processing of long sequences of image pairs in a computationally efficient manner, thereby providing a viable, practical tool for the study of complex flows. They can now resolve the transient 3-D evolution of the complex flows during the majority of the intake stroke of an IC engine-like geometry; in this case all three velocity components are of the same order of magnitude. They are also investigating fluid mechanics inside compact heat exchanger geometries at low Reynolds numbers. These techniques form the starting point for another task in the current collaborative EM Science project: the development of a new Individual Particle Tracking (IPT) method for the laGrangian treatment of much larger flow regions than previously studied.

The University of Idaho has applied laser Doppler velocimetry and flow visualization in conjunction with refractive-index-matching to measure laminar and turbulent flow fields in models of abdominal aortic aneurysms [Budwig et al., 1993]. (The benefit of the Matched-Index-ofRefraction technique is that it permits optical measurements to determine flow characteristics in passages and around objects to be obtained without locating a disturbing transducer in the flow field and without distortion of the optical paths.) An aqueous solution of potassium thiocyanate and ammonium thiocyanate was used to match the index of refraction of an acrylic plastic model. For the flow visualization, solution density was adjusted to match the density of the plastic particles used. They have also employed index-matching for planar laser induced fluorescence measurements (PLIF) of levitated droplets [Budwig, 1994]; refractive index and density were matched between the fluids of both the droplet and the continuous phase.

Low-Reynolds-number turbulent flow is the term employed to describe the range where the fluid and thermal behavior diverges from high-Reynolds-number asymptotic functions [McEligot, Ormand and Perkins, 1966; Patel and Head, 1969]. In circular tubes the Reynolds number range is approximately from 2500 to $10^{4}$. In this range, the velocity distributions are now reasonably predictable provided the flow is fully turbulent (or laminar); the assumed turbulence behavior which is the basis of the velocity predictions also leads to acceptable agreement with measured heat transfer parameters in some cases. Corino and Brodkey [1969] employed refractive index matching and an early, manual version of particle tracking to deduce the instantaneous turbulence structure. While Mikielewicz [1994] demonstrated that a number of popular turbulence models produce poor predictions in this regime, he also showed that the k- $\varepsilon$ model of Launder and Sharma [1974] is satisfactory. For the complex turbulent and laminarizing flows induced by strong heating of a gas flow in this range, several turbulence models have been shown to give good predictions [Kawamura, 
1979; Ezato et al., 1999; Nishimura et al., 2000]. Satake [1996] has extended his Direct Numerical Simulation technique to flow in a circular tube [Satake and Kunugi, 1998].

For another low-Reynolds-number turbulent situation, McEligot and his colleagues have been measuring laminarization of gas flows due to strong heating in longitudinal flow through tubes and ducts [McEligot, Coon and Perkins, 1970; Perkins, Schrade and McEligot, 1973; Shehata and McEligot, 1998] and have been successfully applying various turbulence models for its prediction [McEligot and Bankston, 1969; Perkins and McEligot, 1975; Nishimura et al., 2000; Ezato et al., 1999]. They have also been obtaining data for the related problem of turbulence structure in the viscous layers of flows with strongly favorable pressure gradients [Chambers, Murphy and McEligot, 1983; McEligot and Eckelmann, 1993]. Direct numerical simulations by Japanese colleagues [Satake et al., 2000] have now been applied to the laminarizing data of Shehata and McEligot.

For the present hypothetical concept, design flow rates are expected to give tube Reynolds numbers between 2500 and 5000. Tabulated LDV data for mean and rms axial velocities are available from Lekakis for pipe flow at $\operatorname{Re} \approx 4130$ [Durst et al., 1996] and Satake et al. [2000] have direct numerical simulations at $\mathrm{Re} \approx 4300$, corresponding to the inlet conditions of Shehata and McEligot [1998]. Otherwise, the only data known in the range of interest are the tabulated mean velocity profiles by H. C. Reynolds with an impact tube at $\mathrm{Re} \approx 3020$ and 4080 [Reynolds, Davenport and McEligot, 1968] and the figures of Patel and Head [1969] at $\operatorname{Re} \approx 2440,2615,2680$, 3000,4060 and 4430 with a pitot tube.

In the range $2000<\operatorname{Re}<3000$ or so, the flow may laminar, turbulent or intermittent depending on the entry geometry and the distance from the entry. Rotta [1956] and Lindgren [1953] performed extensive experiments on the flow through tubes at Reynolds numbers in and around the transition region. These investigators examined the behavior at several stations along the tube rather than at one alone. They were thus able to show that the transition from a laminar-flow pattern to turbulent flow at a given Reynolds number occurred continuously as the axial distance increased. Rotta showed that the intermittency factor, $\gamma$ (ratio of time of fluctuating velocity to total time of measurement), increased slowly with axial distance at $\operatorname{Re}=2300$, but increased much more rapidly at higher values. For $\mathrm{Re}=2600, \gamma$ reaches 0.95 at about 370 diameters. The photographic studies of Lindgren qualitatively confirm Rotta's trends. With a hot wire sensor on the centerline of a circular pipe, probably at about 200 diameters, Patel and Head [1969] observed the development of turbulence. Bursts were first observed at $\operatorname{Re} \approx 2000$. The frequency of occurrence of these bursts increased with Reynolds number so it was difficult to determine a precise value at which a fully 
turbulent signal is first obtained. Patel and Head suggested that the intermittency disappears $(\gamma--->$ 1) when $\mathrm{Re}$ is in the region of 3000. On the other hand, Ekman [1910] succeeded in maintaining laminar flow up to $\mathrm{Re} \approx 40,000$ by providing an inlet which was made exceptionally free from disturbances [Schlichting, 1979].

The outflow from the nominally axisymmetric wall boundary layer that results from the impinging jet in the present geometry, in turn, provides the initial conditions for the flow through the array of fuel elements. For predictions through the array, one needs the initial mean velocity and turbulence distributions. As the flow expands radially it decelerates, normally an unstable situation which can be expected to lead to maintenance of a turbulent flow and possible transient, asymmetric separation or flow reversal. For the typical geometry studied in the present work, the extent of the radial flow is of the order of ten times the exit diameter of the central tube.

The literature on heat transfer and flow of impinging jets is vast and growing [Martin, 1977; Downs and James, 1987; Hrycak, 1981; Webb and Ma, 1995; Ma et al., 1997; Lee and Lee, 2000]. The primary application has been for enhancement of convective heat transfer parameters [Gardon and Akfirat, 1965] so much of the literature concentrated on integral heat transfer quantities, such as local or average heat transfer coefficients (e.g., Goldstein and Behbahani [1982], Hrycak [1983], Baughn and Shimizu [1989], Liu and Lienhard [1993], San, Huang and Shu [1997], Ma et al. [1997], Siba et al. [1998], Lee and Lee [2000]) . Numerical predictions are provided by Wolfstein [1970], Rubel [1980], Huang, Mujumdar and Douglas [1984], Amano and Brandt [1984], Craft, Graham and Launder [1993], Dianat, Fairweather and Jones [1996], Heyerichs and Pollard [1996], Lee et al. [1997], Olsson and Fuchs [1998], Satake and Kunugi [1998], Craft [1998], Morris, Garimella and Fitzgerald [1999], Parneix, Behnia and Durbin [1999], Behnia et al. [1999] and others. To maintain effectiveness, often arrays of impinging jets are employed with small pitch-to-diameter spacings, so the non-dimensional radial extent is not large [Womac, Incropera and Ramadyani, 1994; Slayzak, Viskanta and Incropera, 1994; Lienhard et al., 1996]. For gas turbine cooling [Simon et al., 1999], the jet is formed by passage through a short orifice passage so the initial velocity profile of the jet is near uniform; this uniform profile is typical of many experiments where the jet is formed from a converging nozzle and of many numerical predictions.

For the present SNF application, data are needed for low-Reynolds-number jets issuing from long tubes (well-developed flow) into a region which is partially confined by a parallel, perforated plate. However, measurements concentrating on the flow itself -- rather than heat transfer -- have been limited and incomplete. Table I summarizes the fluid physics references found for axisymmetric impinging jets in general; none (except the present study) addresses the combined 
features of this application. (In the table, the term "semi-infinite" refers to jets impinging on flat surfaces in large surroundings and "confined" implies outflow between two parallel surfaces.) With the exception of the outflow study of Moller [1963], the jets from fully-developed flows were measured in semi-infinite surroundings [Cooper et al., 1993; Kim, Wiedner and Camci, 1995; Lee and Lee, 2000]. The early studies of confined flows, such as those of Kreith and colleagues, concentrated on the outflow at large distances from the impinging jet. The confined measurements of Garimella and coworkers are closest to the current interest but their jet source was an orifice. One sees there have been few "low"-Reynolds-number investigations.

At $\operatorname{Re} \approx 2500$, Nelson [1987] employed an LDV to obtain phase-averaged velocity measurements to map coherent spatial characteristics of a flow from a nozzle four diameters above the plate. Landreth and Adrian [1990] used particle image velocimetry to obtain instantaneous velocity fields and averaged quantities for a jet at $\mathrm{Re} \approx 6600$ situated four diameters above their plate; however, their field extended only about two diameters from the centerline. Cooper et al. [1993] conducted a relatively wide range of measurements focusing on data from a long tube to assist turbulence modeling. At $\operatorname{Re}=2.3 \times 10^{4}$ and $7 \times 10^{4}$, they obtained data for a jet located two to ten diameters above the plate to a radius of about eight diameters from the centerline. Results include mean and fluctuating velocities and turbulent shear stresses. They comment that, while the topic has been the subject of many experiments, none could have been said to have been designed with the needs of turbulence modeling in mind. Usually the flow conditions at the nozzle exit are insufficiently prescribed or too far above the plate to be useful. The present objectives include understanding the turbulent or laminarizing flow field approaching the perforated plate, i.e., its upstream conditions (which correspond to the edge conditions of the wall boundary layer from the impinging jet). Even for confined jets, only Fitzgerald and Garimella [1996] appear to have measured turbulence quantities outside the impinging jet and its wall jet; they present rms radial velocity fluctuations, primarily in and near the wall jet at high Reynolds numbers. Emphasis on the region outside the wall jet is lacking in their study.

Numerical studies show that impinging jet flows are difficult to predict reliably. Craft, Graham and Launder [1993] attempted four turbulence models -- one k- $\varepsilon$ model and three second-moment closures -- and found none to be entirely successful. Morris, Garimella and Fitzgerald [1999] showed a standard high-Re k- $\varepsilon$ model and an RNG k- $\varepsilon$ model to be inferior to a Reynolds stress approach. Behnia et al. [1999] indicated that the flow characteristics at the jet inlet strongly affect the heat transfer rate (so the use of the appropriate jet velocity profile is important for applications). Advanced numerical techniques are beginning to be employed. Olsson and Fuchs [1998] studied the use of large eddy simulations (LES), examining the effects of spatial resolution and different sub- 
grid-scale models. Satake and Kunugi [1998] extended an axi-symmetric direct numerical simulation (DNS) technique to treat approximately the confined jet flow experiment of Nishino et al. [1996] at a Reynolds number near 10,000. Provided spatial resolution is adequate, DNS can be a means to avoid problems with turbulence modeling but computational requirements are extensive even for low-Reynolds-number flows.

In addition to biomedical applications [Lieber, Stancampiano and Wakhloo, 1997] and chemical processing, the flow through perforated plates and screens is of interest for flow conditioning in wind tunnels [Farell and Youssef, 1996; Watmuff, 1997] and in setting the initial conditions of film cooling jets in turbine blades and rocket nozzles [Simon et al., 1999]. The effects of the flow resistance provided by a perforated medium, such as a gauze screen, on mean velocities, fluctuations and directions have been treated for wind tunnel applications by Prandtl [1933], Taylor [1944], Taylor and Batchelor [1949] and Pankhurst and Holder [1952]; non-uniformities can be decreased or increased, depending on the resistance coefficient [McEligot, 1981]. Bradshaw [1965] concluded that spanwise variations in wind tunnels were produced by a spatial instability of the flow through the screens when the open-area-ratio was too small; fluid emerges from the screen as a pattern of jets which tend to stick together because they can only entrain fluid from each other. Much of the information on effects on turbulence can be found in a review by Laws and Livesey [1978]. Resistance to flow through barriers uniformly distributed over a channel cross section is treated extensively by Idelchick [1994]. Tan-Atichat, Nagib and Loehrke [1982] measured the dependence of some perforated plates as turbulence manipulators. Most of the film cooling investigations of flow through perforated plates have concentrated on measuring various effects on the discharge coefficients of the holes [Rohde, Richards and Metger, 1969; Hay, Lampard and Benmansour, 1983; Hay, Henshall and Manning, 1994; Gritsch, Schulz und Wittig, 1997; Burd and Simon, 1998]. Burd and Simon [1997] measured the influence of coolant supply geometry on coolant exit flow and Kohli and Thole [1997] applied a CFD calculation for the effects of entrance crossflow directions to film cooling holes. Hale, Plesniak and Ramadhyani [1999], Gritsch, Schulz und Wittig [1999] and Wilfert and Wolff [1999] examined the influence of holes and internal flow on film cooling effectiveness.

In the current study the velocity and turbulence distributions vary radially across the entrance to the generic "array of tubes" (representing fuel elements). The flow direction would be expected to be predominantly longitudinal but the velocity distribution coming from the perforated plate is highly non-uniform and crossflow exists as the flow adjusts towards a fully-developed condition and then radially converges towards the exit from the vessel (see section on INEEL studies later). The low Reynolds number involved is expected to induce laminarization, i.e., a decaying level of 
turbulence, both longitudinally and radially. A useful reference for heat transfer in low Reynolds number flow through and across rod bundles is the collection by Kakac, Shah and Bergles [1983], with the emphasis on laminar flow. Todreas and Kazimi [1990] provide brief summaries concentrating on application to nuclear reactors and Kakac and Spalding [1979] include thirteen papers relating to rod bundles in the proceedings of their conference in Istanbul.

Heat transfer and friction parameters for cross flow through bundles have been available for many years for a wide range of arrays [Grimison, 1937; Kays and London, 1955; Zukauskas, 1972]. Pressure drop and local heat transfer coefficients were obtained by Achenbach [1989] for staggered tube bundles at high Reynolds numbers. Velichko, Pronin and Yassin [1993] measured heat transfer and friction parameters for non-traditional tube bundle arrangements. Stanescu, Fowler and Bejan [1996] determined optimal spacing of the cylinders to maximize the average heat transfer conductance for an entire bundle. Barsamian and Hassan [1996] have applied large eddy simulation (LES) to spatially-periodic flow across a tube bundle at $\mathrm{Re} \approx 1.7 \times 10^{5}$; fluctuating lift and drag spectra were calculated and compared to the limited data available [Chen and Jendrzejczyk, 1987]. Mean and fluctuating velocity components were measured at $2 \times 10^{5}<\operatorname{Re}<2 \times 10^{6}$ by Poskas and Survila [1983] for staggered and in-line bundles of tubes. Katinas et al. [1990] measured tube vibrations induced at turbulence levels from one to twelve per cent in the upstream flow to staggered and in-line tubes; although the Reynolds number supposedly was varied from $10^{3}$ to $2 \times 10^{5}$, effects were not presented in terms of Reynolds number. Fluctuations of heat transfer and velocity have been measured in a staggered tube array by Scholten and Murray [1998]. Beale and Spalding [1998] numerically predicted laminar flow and heat transfer across a variety of tube bundle geometries. Watterson et al. [1999] applied a low-Reynolds-number k- $\varepsilon$ model to flow around an array of staggered tubes. A k- $\varepsilon$ model was also applied by Wilson and Bassiouny [2000] to predict the laminar and turbulent flow of air across tube banks with apparently satisfactory agreement to data.

In the present application, the longitudinal Reynolds number (based on hydraulic diameter) is expected to be of the order of 100 in the fuel element array. Predictions for longitudinal flow typically consider the situation as fully-developed flow through a non-circular channel. Rehme [1992] has reviewed the measurements of turbulence structure in longitudinal flow through rod bundles and related approaches to treating mixing between subchannels; he concentrated on conditions for Reynolds numbers of the order of 5 x 104. Rowe, Johnson and Knudsen [1974] measured axial velocity and turbulence intensity and a few data on lateral turbulence intensity in fully-developed flow through rod bundle channels at Reynolds numbers from $5 \times 10^{4}$ to $2 \times 10^{5}$. Ouma and Tavoularis [1991] measured mean velocity, Reynolds stresses and turbulent scales in fully-developed flow through a bundle including rod-wall subchannels; the Reynolds number was 
about $4.8 \times 10^{4}$. For axial flow at Reynolds numbers of $6 \times 10^{4}$ to $1.4 \times 10^{5}$ to determine cross-flow mixing factors in several arrays, Möller [1992] measured axial velocity distributions, wall shear stresses, Reynolds stresses, wall pressure fluctuations and spectra of axial and azimuthal components of fluctuating velocity; he concluded that the process of cross-flow mixing was related to a phenomenon of quasi-periodic flow pulsations across gaps between adjacent subchannels. A threewire probe was employed by Krauss und Meyer [1998] to measure all Reynolds stresses and heat fluxes in the turbulent flow through a central channel of heated rod bundles. Prithiviraj and Andrews [1998] have developed a complicated, three-dimensional numerical simulation for shelland-tube heat exchangers and claim good agreement with measured pressure drops. Turbulent mixing in rod bundles has been predicted by Kim and Park [1998] with an anisotropic diffusion model.

The fundamental problem of flow field readjustment as turbulence fluctuations decrease in flow through a rod bundle at a low Reynolds number does not appear to have been addressed. This "laminarizing" process is a key phenomenon which should be investigated. Mikic, Vujisic and Kapat [1994] have proposed a new hypothesis for transition to turbulent flow and maintenance of turbulence in channel flow; it is expected that such a study would help assess this hypothesis from an opposite viewpoint, "reverse transition" to laminar flow.

\section{Scientific needs}

As noted, despite the wide range of previous work on individual fluid physics phenomena occurring in these generic problems, a number of scientific needs remain. These needs lead to the objectives and tasks being attacked in the present project.

New fundamental experimental measurements needed include

- Determination of the decay of turbulent fluctuations in the outflow of a low-Reynolds-number impinging jet along a semi-confined wall

- Determination of the decay of turbulence (or "laminarization") in flow around a rod bundle

- Determination of the overall flow pattern and time-resolved distributions of mean velocity components and turbulent fluctuations from the central inlet tube through the vessel 
- Determination of typical paths of (simulated) oxidizing molecules from introduction until interaction with a surface

\section{Engineering needs}

To develop useful, reliable predictive techniques for describing flow and turbulence fields plus related heat and mass transfer in applications to SNF canisters in general,

- Computer codes available or proposed to address heat and mass transfer in SNF canisters must be assessed by comparison to careful measurements -- at the (non-dimensional) conditions of the applications -- of the fundamental phenomena described above

- Suitable computer codes must be improved or developed to treat successfully any significant discrepancies observed in the comparisons, using the fundamental measurements for guidance and

- Resulting computer codes or supporting subroutines must be verified by successful comparison to benchmark measurements at conditions corresponding to the ranges of parameters in the applications.

The role of the INEEL contribution is to provide fluid physics measurements addressing these needs. 


\section{INEEL STUDIES}

To date the INEEL efforts have emphasized two tasks:

- Overall flow visualization for a variety of configurations

and

- Pointwise turbulence and velocity measurements with two-component laser Doppler velocimetry in the unique INEEL Matched-Index-of-Refraction flow system

\section{Generic idealization}

As noted in the introduction, a wide variety of fuel element geometries are accommodated in the storage of spent nuclear fuel. The fuel may have high-, medium- or low-enrichment so criticality considerations affect the number of fuel elements that can be stored in a single fuel basket or canister. Typically, only a few high- or medium-enriched elements can be stored in one basket whereas fuel elements with low-enrichment can be packed densely, approaching the appearance of arrays in a shell-and-tube heat exchanger. Element shapes may be circular rods, annuli, plates, squares, "trapezoids," hexagons, etc. (Figure 1). Proposed canister sizes range from 14 to 24 inches (36 to $60 \mathrm{~cm}$ ) with lengths of ten to fourteen feet (3-4 m). Typically, elements or assemblies are placed in baskets for dry storage, with drain holes in the basket support plates; the hole pattern gives an open-area-ratio of three to nine per cent, depending on the design. The number of baskets vertically in a canister depends on the lengths of the fuel elements being stored. Consequently, there is no one generic fuel element / assembly and, therefore, no single generic fuel storage configuration.

One can, however, identify generic flow phenomena and paths. A generic idealization of a combined drying and passivation (surface oxidation) approach has been defined, representing a section of a vertical cylindrical canister containing baskets of SNF elements. This simulation includes flow phenomena that occur in canisters for high-enrichment and medium-enrichment fuels, where fuel element spacing in the canister is increased as compared with low enrichment fuel. A steady flow of helium plus oxygen passivation gas is introduced at the bottom of the canister via a central tube from the top. Fluid spreads radially near the (circular) bottom canister wall and then flows through an array of holes in the perforated basket support plate. Fluid then flows around the elements and out the top. Dimensions and flow rates for the idealized situation are within the ranges 
for typical drying canisters. Approximate calculations have identified the ranges of values of flow parameters needed to determine the flow regimes occurring in practice.

With Prof. John C. Crepeau of the University of Idaho, an idealized description has been developed for a geometry which induces the generic flow phenomena expected in typical SNF canisters, with provision for inflow to simulate purge gas flow including an oxidizing component for passivation of uranium hydride surfaces. Initial models correspond to sparse loading of simulated elements as for highly- and moderately-enriched fuels. A perforated plate is employed to simulate characteristics of flow through the support plates of baskets in canisters. The idealization (Figure 3) was chosen to have a canister diameter of 18 inches $(46 \mathrm{~cm})$ and a basket length of about four feet $(1.3 \mathrm{~m})$. A long "dip tube" of one inch diameter $(2.5 \mathrm{~cm})$ serves as the inlet for the drying / passivation gas. It is recognized that current canisters may not have this configuration but comparable passages for circulation have been suggested in some proposed designs. Consequently, comparable generic phenomena could be expected to occur. Suggested design flow rates for the earlier hypothesized application indicate that the Reynolds number in the inlet tube would be expected to be between 2500 and 5000, i.e., relatively low. The distance between the basket support plate and the bottom of the canister was taken as two inches $(5 \mathrm{~cm})$. For convenience in physical and numerical modeling, while retaining the key flow phenomena, symmetry and circumferential periodicity were employed to the extent possible. 
For this idealization, at $\mathrm{Re}=2500$ the governing non-dimensional parameters would approximately be
- Inlet circular tube
$\operatorname{Re} \approx 2500$
$\mathrm{L} / \mathrm{D} \approx 80$

- Axisymmetric impinging jet

$\mathrm{Re}_{\mathrm{jet}} \approx 2500$

$\mathrm{s} / \mathrm{D} \approx 2$

$\mathrm{H} / \mathrm{D} \approx 2$

$\mathrm{R} / \mathrm{D} \approx 6$

- Perforated bottom support plate Rehole $\approx 40-160 \quad \mathrm{ph} /$ Dhole $\approx 4 \quad \mathrm{t} /$ Dhole $\approx 1$ Approach angle -- unknown

- Array of fuel elements

$$
\begin{aligned}
& \text { ReD, h,avg } \approx 70 \quad \mathrm{~L} / \mathrm{Dh}_{\mathrm{h}} \approx 6 \quad \mathrm{p} / \mathrm{D}_{\text {element }} \approx 2 \\
& \mathrm{~L}^{+} \approx 0.4 \quad \text { "Equivalent" } \mathrm{K}_{\mathrm{V}} \approx 0.02 \quad \mathrm{~K}_{\mathrm{p}} \approx-0.11 \\
& \text { Inlet turbulence -- unknown } \quad \text { Angle of flow -- unknown }
\end{aligned}
$$

\section{Objective}

The general (long term) goal of this collaborative program is to develop reliable predictive techniques for the energy, mass and momentum transfer plus chemical reactions in drying and passivation operations in the transfer of Spent Nuclear Fuel (SNF) from wet to dry storage. Such techniques are needed to assist in design of future transfer and storage systems, prediction of the performance of existing and proposed systems and safety (re)evaluation of systems as necessary at later dates. The goal of the present project is to provide the scientific knowledge required to develop and to assess numerical predictive methods treating the key flow phenomena in generic simulations of drying / passivation problems in Environmental Management. The objective of the INEEL tasks is to obtain fundamental measurements of the basic velocity and turbulence fields in generic idealizations of flow processes in SNF canisters during transfer and/or storage; as noted, proposed operating conditions give typically low-Reynolds-number flows.

\section{Choice of experiments}

As noted earlier, the expected range of inlet Reynolds number is about 2500 to 5000 for the potential applications. However, in the range $2000<\operatorname{Re}<3000$ or so, the flow may laminar, turbulent or intermittent depending on the entry geometry and the distance from the entry [Rotta, 1956; Lindgren, 1953; Patel and Head, 1969]. So at the lower end of the expected application range, the flow state at the exit of the tube (the jet) would be uncertain unless experiments were 
performed with the identical inlet geometry and flow rate. (At some time in the future direct numerical simulations [Moin and Mahesh, 1998] may be able to predict this flow state adequately.)

Two experiments were designed and conducted. The first was aimed at providing overall flow visualization for a fully-developed, turbulent entering flow. As a consequence of its simplicity, it was possible to obtain data for several flow configurations of interest. The second was a more detailed design to take advantage of the unique INEEL Matched-Index-of-Refraction flow system and its two-component laser Doppler velocimeter for pointwise velocity and turbulence measurements. The inlet flow configuration selected could be expected to provide an intermittent flow, undergoing transition from turbulent to laminar [Patel and Head, 1969]. Consequently, the present study is expected to provide two inlet flows representing the range of conditions likely at the lower Reynolds numbers. While the second apparatus was designed for several configurations, only one configuration could be employed in the time available due the required duration of the time series measurements at each measuring location. For both experiments, most but not all measurements were obtained for $\mathrm{Re} \approx 2500$.

\section{Overall flow visualization}

Corroded spent nuclear fuel (SNF) elements may be passivated by injecting treatment gases into a storage canister containing the elements. For effective passivation, a design aim would be controlled, moderately high mass transfer rates to the fuel elements. These rates would depend on the flow pattern and turbulence levels in the vicinity of the elements. By developing a reliable predictive technique for the energy, mass and momentum transfer in the presence of surface reactions, transfer and storage systems can be efficiently and safely designed. An overall objective of the research program described in this report is to understand the fluid physics of the passivation process. This objective was pursued by using flow visualization techniques and experimental measurements of the flow field to support computational models.

A water-flow experiment with a 3/4-scale model (relative to the idealized canister) has been used for overall flow visualization and velocity measurements, with and without an array of simulated fuel elements. Its purposes were to obtain initial indications of the gross flow behavior and to investigate the circumferential periodicity of the flow. Standard flow visualization techniques, using fluorescent dyes and neutrally buoyant particles illuminated by laser and white light, were employed. Observations have been made with perforated plates (representing basket support plates) having three hole geometries, with and without simulated fuel elements. 
Experiment design and procedures Flow visualization experiments were performed in a transparent, approximately 3/4-scale geometric model of our idealized canister in a water loop. The apparatus is shown schematically in Figure 4. The cylindrical canister contains four simulated fuel element tubes symmetrically distributed. The region contained between the bottom plate of the simulated canister and simulated bottom plate of a basket will frequently be called the "inlet plenum" or plenum region since it has some features of the plenum of a shell-and-tube heat exchanger or a nuclear reactor. The inlet plenum width is twice that of the inlet pipe diameter. The interface between the inlet plenum and the simulated fuel element bundle consists of a perforated (porous) plate, which simulates a basket support plate. Three geometries of simulated basket support plates with $50 \%, 8 \%$, and $4 \%$ porosity were used. The $4 \%$ and $8 \%$ porosity plates lie within the $3 \%$ to $9 \%$ range of porosities of typical storage canisters. Plate perforations consist of holes drilled in a square lattice array with $1.90 \mathrm{~cm}$ spacing between holes. The spacing is threequarters that of the idealized prototype. The $50 \%$ porosity plate was used to investigate the effects of plate porosity on the flow field further.

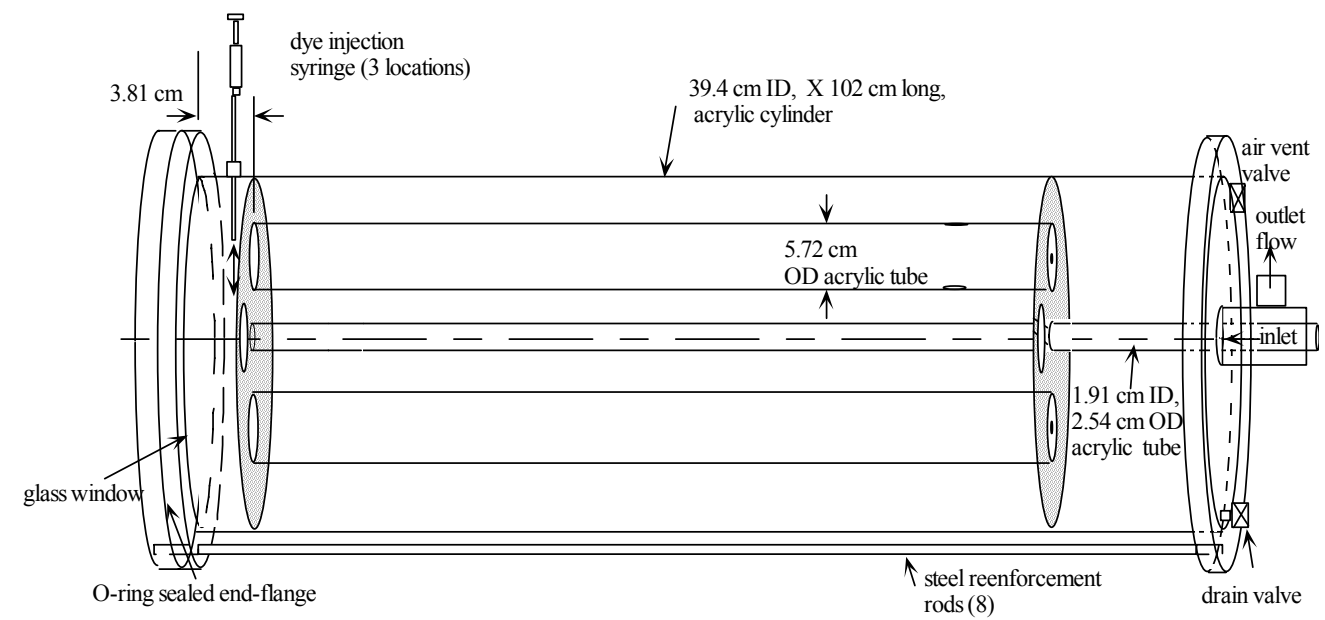

Fig. 4. Schematic diagram of apparatus for flow visualization.

The inlet flow to the apparatus is provided by gravity feed from an approximately 60 liter reservoir which is elevated about one meter above the apparatus inlet. Flow from the apparatus is collected in a separate reservoir where it is pumped continuously back to the inlet reservoir. Gravity feed and pump flow rates are controlled by valves. Reynolds number scaling of flow rate to the prototype is employed. The experiments reported employ an inlet tube Reynolds number of $2,500 \pm 100$. 
Due to the low velocities in the apparatus the fluid temperature profile in the flow loop must be close to isothermal if currents induced by natural circulation are to be avoided. The induced velocity is small in comparison with forced convection if the Richardson number $R i=g \alpha \Delta T L / V^{2}$, the ratio of buoyant to viscous forces, is significantly less than one. Because inlet fluid is effectively mixed with fluid in the apparatus in the inlet plenum, it is difficult to prescribe an effective $\Delta \mathrm{T}$. It was observed from flow visualization that fluid in the apparatus and reservoirs must be within approximately $1{ }^{\circ} \mathrm{C}$ to avoid noticeable distortion of the flow pattern. The fluid is typically within $0.2^{\circ} \mathrm{C}$ of ambient temperature during experiments. The small temperature difference is due to pumping power and friction. The apparatus and reservoirs are filled several days before experiments are conducted to achieve near isothermal conditions. This waiting period also insures that the water is well deaerated so that bubbles do not leave solution and attach to walls (using Kodak Photoflo surfactant also helps prevent bubble formation and attachment). Bubbles are more of a problem for light scattering than for flow perturbation.

Flow visualization was performed using fluorescent dye and neutrally buoyant particles with laser light sheet illumination. A typical configuration for laser illumination is shown in Figure 5. The configuration for white light illumination is similar to that for laser illumination except that a collimated light source and a slit are substituted for the laser and light sheet generator. Collimated laser or white light sheets were also configured to bisect the inlet plenum and downstream of the perforated plate perpendicular to the flow axis. An example photograph of inlet plenum flow is shown in Figure 6 which shows a cross-section of an inner ring vortex (compare with later Figures 8 and 9). For the photograph, Rhodamine WT fluorescent dye was illuminated with a light sheet generated by an argon-ion laser. The light sheet approximately illuminated a plane midway between the perforated plate and the wall (window).

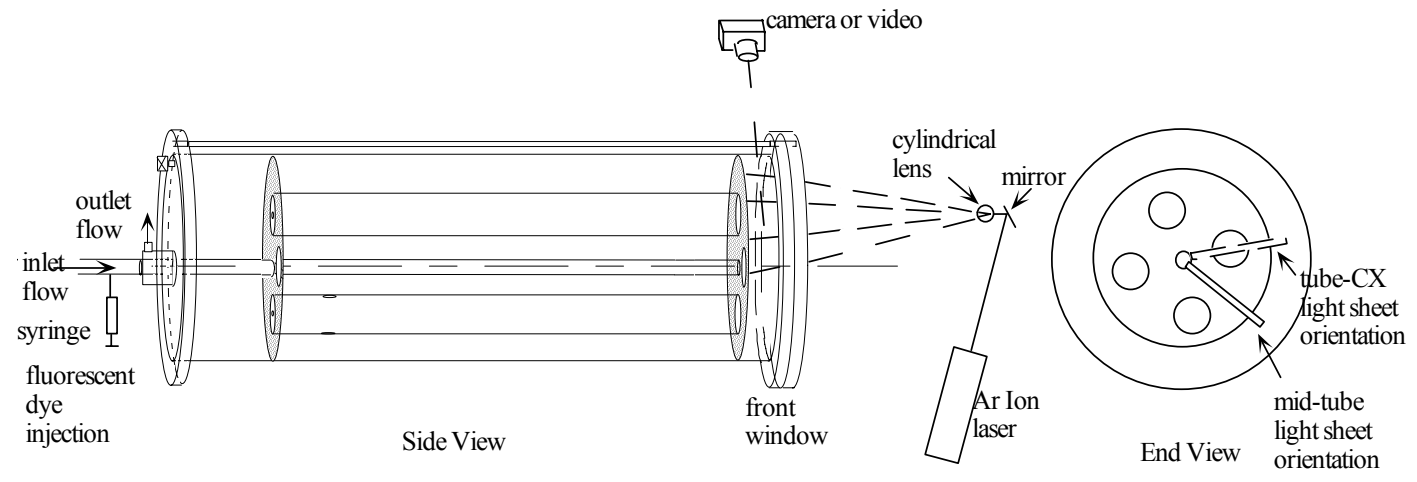

Fig. 5. Schematic diagram of illumination for flow visualization using a laser light sheet. 


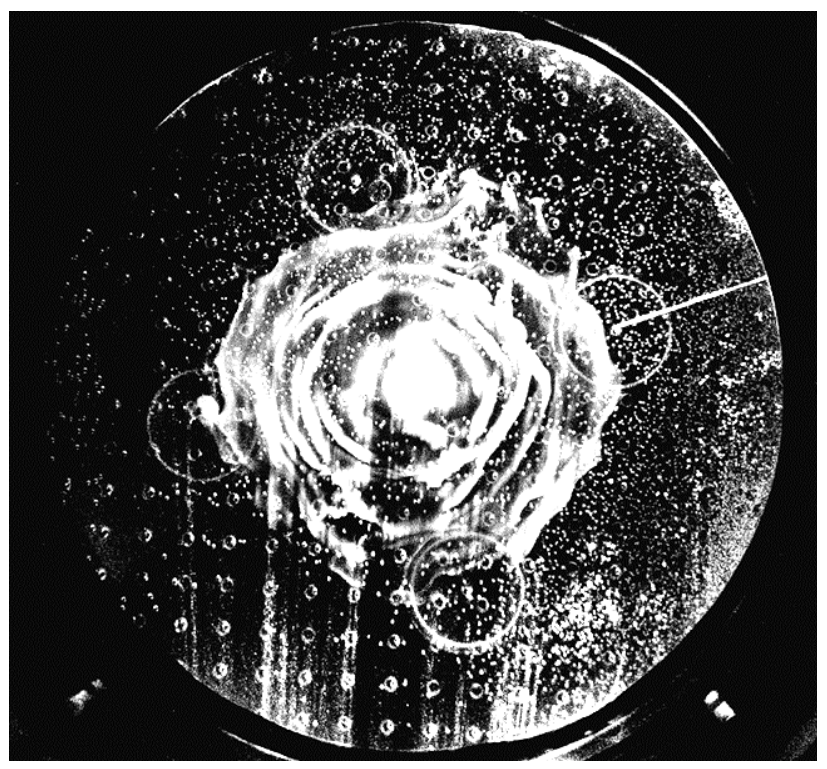

Fig. 6. Visualization of vortex formed in the region contained between the bottom plate of the simulated canister and simulated bottom plate of a basket ("inlet plenum").

The motions of neutrally buoyant particles were tracked from long duration photographs and from video recordings. The photographs and video images were then analyzed to provide fluid velocity information. The procedure for extracting two-dimensional, radial and axial, velocity information from particle pathline photographs is as follows:

1. Photograph a dilute cloud of particles using collimated white light sheet illumination with sufficient sheet width $(0.5 \mathrm{~cm}$ is typical $)$ to insure that the majority of particles do not wander out of the beam during the exposure. A typical exposure duration is from $1 / 2 \mathrm{~s}$ for flow in the inlet plenum to $6 \mathrm{~s}$ for flow downstream of the perforated plate.

2. Scan enlarged prints of the negatives into Canvas software (Deneba Software Inc.).

3. Correct for distortion caused by refraction from photographing at an acute angle through a cylindrical wall. This process was done by linearly scaling to provide the same cylinder inside radius as equal axial length.

4. Trace particle pathlines on a separate image layer in Canvas. Use flow visualization information and video recordings to determine velocity direction. Discard photograph layer.

5. Transfer velocity vectors to a cross-section drawing of the apparatus. 
Another technique employed for extracting velocity information was to capture video images of the neutrally buoyant particles using a RaasterOps frame grabber board in a computer and then overlaying images for known time intervals. This technique was successful for characterizing the higher velocity flow field outside the wall jet in the inlet plenum. The velocity in the wall jet was too high to track individual particles between frames taken at 1/30 s intervals. Particle path line photography proved to be more successful outside the higher velocity eddies in the inlet plenum and downstream of the perforated plate. The much higher resolution of $35 \mathrm{~mm}$ black and white film compared to video images permitted a wider field of view for particle tracking.

The neutrally buoyant particles were silver coated hollow glass spheres of approximately fourteen microns in diameter. These spheres are available from TSI Incorporated. The spheres exhibit a fairly broad range of densities. This density variation would not be a problem for turbulent flow. However, for low-Reynolds-number laminar flow the spheres must be more closely neutrally buoyant. Near-neutral buoyancy was achieved by mixing a batch of spheres with water in a beaker and waiting several hours. The majority of the particles settle to the bottom and many float on the surface. The remaining particles in suspension (calculated to be less than one per cent of the total) plus water were removed using a hypodermic syringe and then injected directly into the apparatus. This method proved to be quite successful in providing the highly reflective neutrally buoyant particles necessary for particle tracking. (One objective of these experiments was to find a suitable particle for PTV experiments proposed for the MIR model. We would use a similar technique to sequester silver coated hollow glass spheres that are neutrally buoyant in the oil used in the flow loop).

Experimental uncertainties The experimental uncertainties associated with the velocity vectors to be shown in Figures 8 and 9 result from a number of sources and vary with position. The sources include (1) uncertainty of visually interpreting path line length, (2) distortion caused by refraction through a cylindrical wall at varying angles to normal and (3) uncertainty associated with the transient shifting of flow patterns. The uncertainty in estimating flow rate is measured to be less than five per cent of measured value and is not significant relative to the uncertainties in velocity vectors.

The uncertainty of interpreting particle path line length is estimated as $\pm 20 \%$ of the measured length. The correction for distortion caused by refraction from photographing at an acute angle through a cylindrical wall was performed by linearly scaling the radial dimension to provide the same cylinder inside radius as equal axial length. This approach does not account for distortion 
due to the uncorrected wall curvature (somewhat similar to spherical aberration in a lens). This distortion increases with the angle from normal to the cylinder wall and was determined from photographs of a rule placed inside the water filled cylinder. The maximum distortion for camera view angles used in the photographs is estimated to be $\pm 20 \%$ of the measured length.

The overall RMS uncertainty [Cook and Rabinowicz, 1963] of determining the velocity vector due to the above sources, without considering the transient shifting of flow patterns, is approximately $\pm 30 \%$. It should be understood that the vectors do not represent an average velocity for their positions. Average and instantaneous velocities could be determined in experiments using Particle Tracking Velocimetry (PTV). This technique would provide more accurate velocity measurements with three-dimensional velocity components rather than the two-dimensional components described in this report.

Results Experiments were conducted to determine the canister flow field with three perforated plates with open areas of 50\%, 8\% and 4\% ("porosities"). Experiments reported are for inlet tube $\mathrm{Re}=2,500 \pm 100$. Although laminar flow in a smooth tube can occur at Reynolds number as high as approximately 40,000, it is believed that the tube is sufficiently long and the entrance conditions sufficiently abrupt to insure that turbulent flow exits the tube and impinges on the plate.

With a $50 \%$ open area plate, the flow pattern is comparable to that of a submerged impinging jet on a semi-infinite plate with formation of a large vortex and entrainment and recirculation through the holes in the perforated plate near the center. Downstream flow is primarily through the outer holes. A single main recirculating eddy forms encompassing the plenum and part of the region for the element array. The flow pattern, as determined from the various flow visualization techniques, is shown in Figure 7.

With the $4 \%$ and $8 \%$ open area plates, recirculation regions occur downstream of the perforated plate and two main recirculation zones (plus a small corner flow vortex) form upstream of the perforated plate. Flow does not reenter the inlet plenum through the plates with less open area as it does with the $50 \%$ open area plate. In these cases the flow pattern in the inlet plenum has some features of a confined impinging jet [Morris and Garimella, 1996]. 


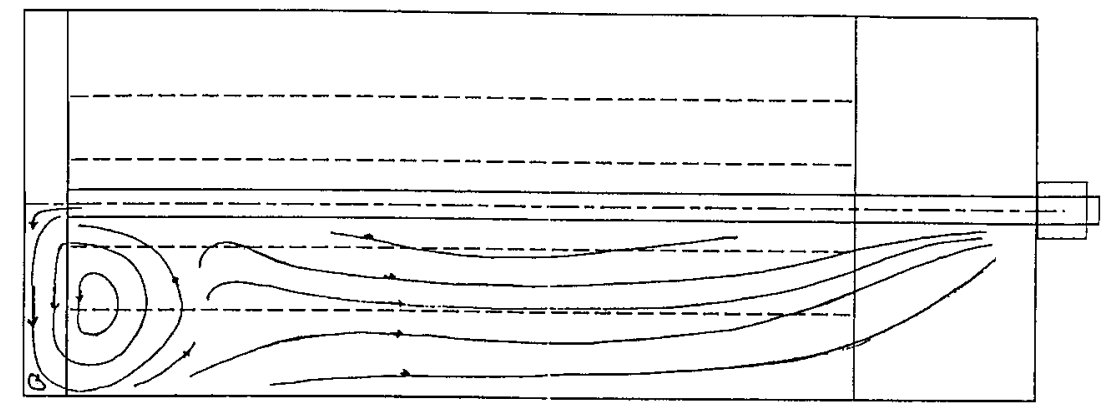

\section{Basket support plate with $50 \%$ open area}

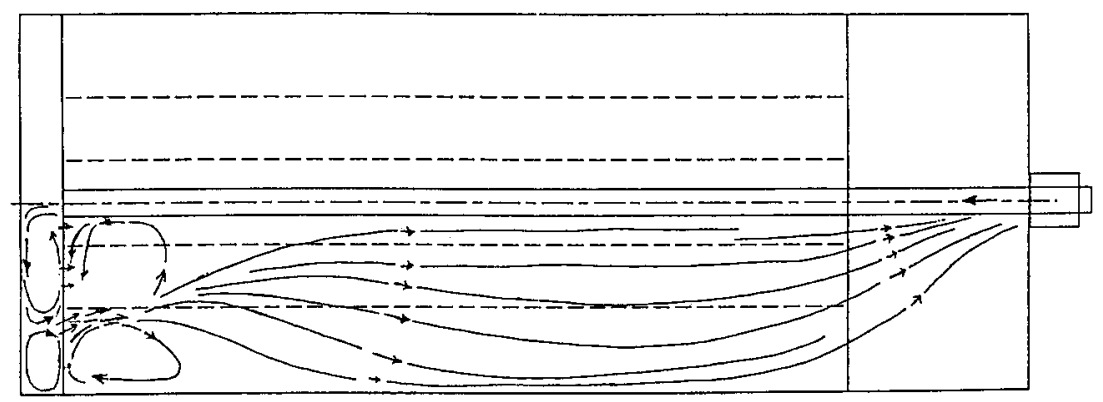

Basket support plate with $4 \%$ open area

Fig. 7. Effects of design of basket support plate on simulated canister flow shown by deduced streamline patterns (a) fifty per cent open area and (b) four per cent open area.

The flow is approximately circumferentially periodic and symmetrical about cross-sections through the four outer tubes for experiments using each of the three perforated plates. This observation implies that numerical predictions and detailed measurements can concentrate on an $1 / 8$-the sector of the idealized geometry rather than the full cross section. The flow is only approximately symmetrical because of time varying asymmetry of flow development in the inlet plenum, shown in Figure 6, and vortices downstream of the 4\% porosity plate that slowly oscillate in position. The positions of the vortex centers may vary by one or two centimeters (approximately $\mathrm{D}_{\text {tube }}$ ) over a period on the order of one minute. Although the vortices are similar in different quadrants, the positions of their centers do not appear synchronized in time.

Flow visualization experiments show tube cross-flow and resultant vortex shedding, although the vortices do not show the clear alternating pattern of a Karman vortex street [Tritton, 1977]. A Reynolds number based on tube diameter of approximately 40 is about the highest number for 
which there is no unsteadiness due to vortex shedding [Tritton, 1977]. This Reynolds number corresponds to a cross-flow velocity of only approximately $0.004 \mathrm{~cm} / \mathrm{s}$ for water flow across the $5.72 \mathrm{~cm}$ diameter outer tubes. The measured tube cross-flow velocities near the $4 \%$ open area plate (Figure 8 ) are position dependent, but are of the order of $0.1 \mathrm{~cm} / \mathrm{s}$, which corresponds to $\operatorname{ReD} \approx$ 1,000 (transition to turbulence occurs at approximately $\operatorname{Re} \mathrm{D}=3 \times 10^{5}$ for a cylinder in cross flow).

The vortex shedding frequency should scale with Strouhal number, $\mathrm{St}=\mathrm{fD} / \mathrm{U}$, a nondimensional frequency. A typical value of St for laminar flow is about 0.2 [Tritton, 1977] although there is no reason to expect one specific value for a non-uniform velocity distribution. Overall the Strouhal number is observed to be a function of Reynolds number [Tritton, 1977]. The observed variations in eddy positions occur with a frequency of approximately one minute with a variation of at least $\pm 30 \mathrm{~s}$. The variation implies that this behavior may be a chaotic process, in the sense that the flow has uncorrelated behavior, exhibiting sensitive dependence on initial conditions [Tufillaro, Abbott and Reilly, 1992]. The three dimensions of fluid motion further the possibility of chaos. (We have not investigated this aspect mathematically.) Strouhal number scaling may be used to estimate the vortex shedding periods to be expected in the MIR model and in the prototype canister, which would be approximately $3 \mathrm{~s}$ and $0.3 \mathrm{~s}$ respectively, assuming Reynolds number scaling of cross-flow velocity for $\operatorname{Re}=2500$. A non-dimensional wavelength $(\lambda / \mathrm{D})$ for the vortices may be expressed as $\lambda / \mathrm{D}=\mathrm{U} / \mathrm{fD}=1 / \mathrm{St}$. If $\mathrm{St}$ is approximately constant in the models and the prototype for the same Reynolds number then the non-dimensional wavelength should also be approximately the same in the model and the prototype. This non-dimensional wavelength preserves the geometric scaling implied in Reynolds number scaling.

Results from experiments using the apparatus containing the inlet tube and the $4 \%$ porosity plate, but with the outer tubes removed, indicate that the vortices downstream of the plate are not solely formed by vortex shedding from the tubes. The circulation pattern within a distance of about one cylinder radius downstream of the plate is similar to the mid-tube cross section flow shown in Figure 9, complete with the vortex near the plate and one vortex downstream of the plate. The two main counter rotating vortices in the inlet plenum shown in Figures 8 and 9 are present in about the same positions. 


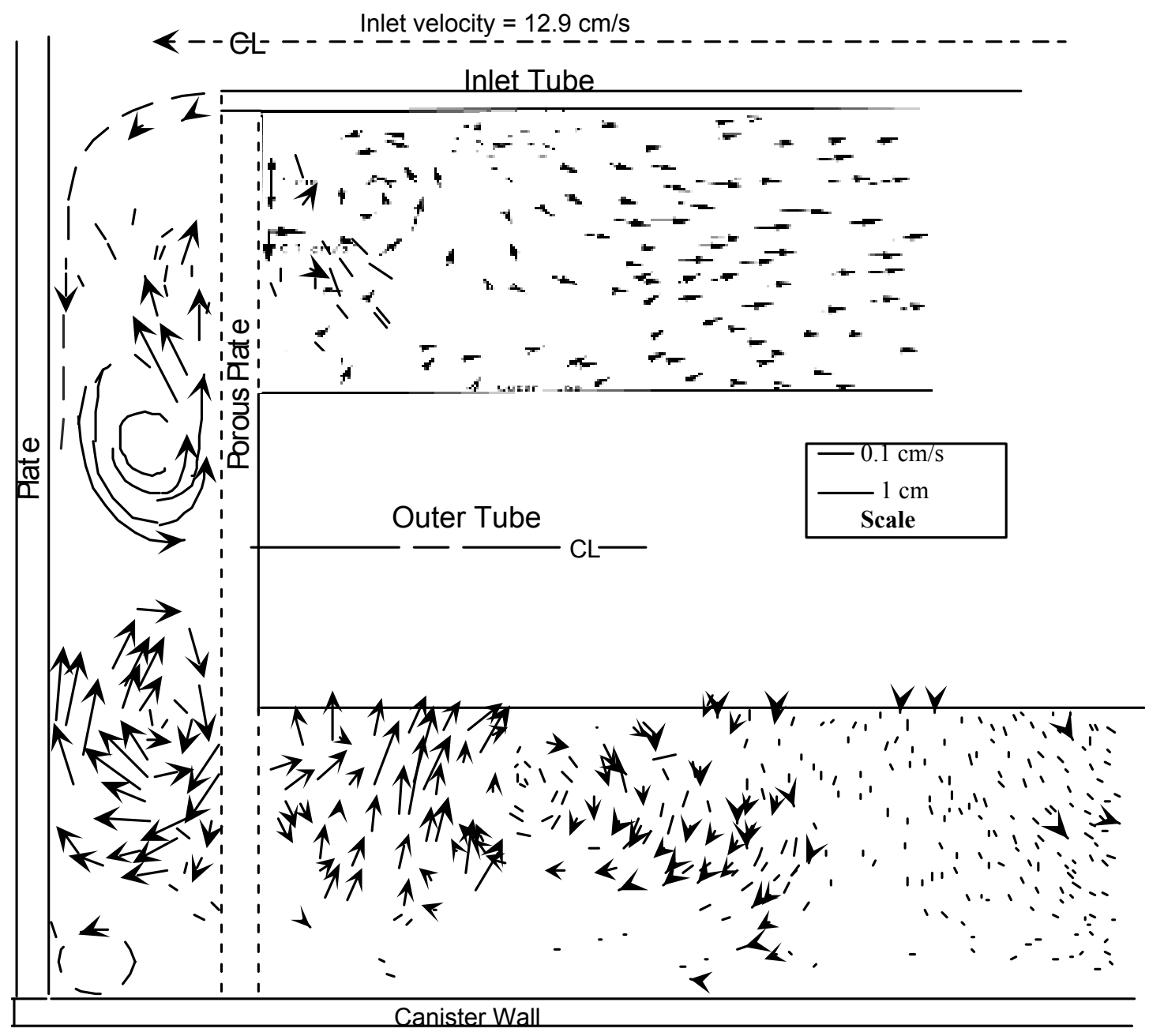

Fig. 8. "Instantaneous" velocity distribution in a plane through the simulated fuel elements, Retube $\approx 2500$.

With the tubes removed, subsequent vortices downstream of the plate as shown in Figure 9 are not present and the flow approaches an approximately parabolic laminar velocity profile within about three cylinder radii downstream of the plate. The fully developed laminar velocity profile for an annulus has the form $U(r)=-r^{2}+A^{*} \ln \{r\}+B$, where $r$ is radius and $A$ and $B$ are constants. This approach to a laminar profile is consistent with the criterion that, for a circular tube flow, fully-developed flow is approximately achieved within about $\mathrm{L} / \mathrm{D}=\mathrm{Re} / 30$ [Smith, 1960]. The Reynolds number in the array downstream of the plate based on hydraulic diameter is approximately 70 for an inlet $R e=2,500$. With the tubes present, vortices persist for the majority of the tube bundle length and the flow only starts to approach a fully-developed laminar profile near the exit of the bundle $\left(\mathrm{L} / \mathrm{Dh}_{\mathrm{h}} \approx 6\right.$, where $\mathrm{Dh}_{\mathrm{h}}$ is the hydraulic diameter $\left.\approx 18.3 \mathrm{~cm}\right)$. 


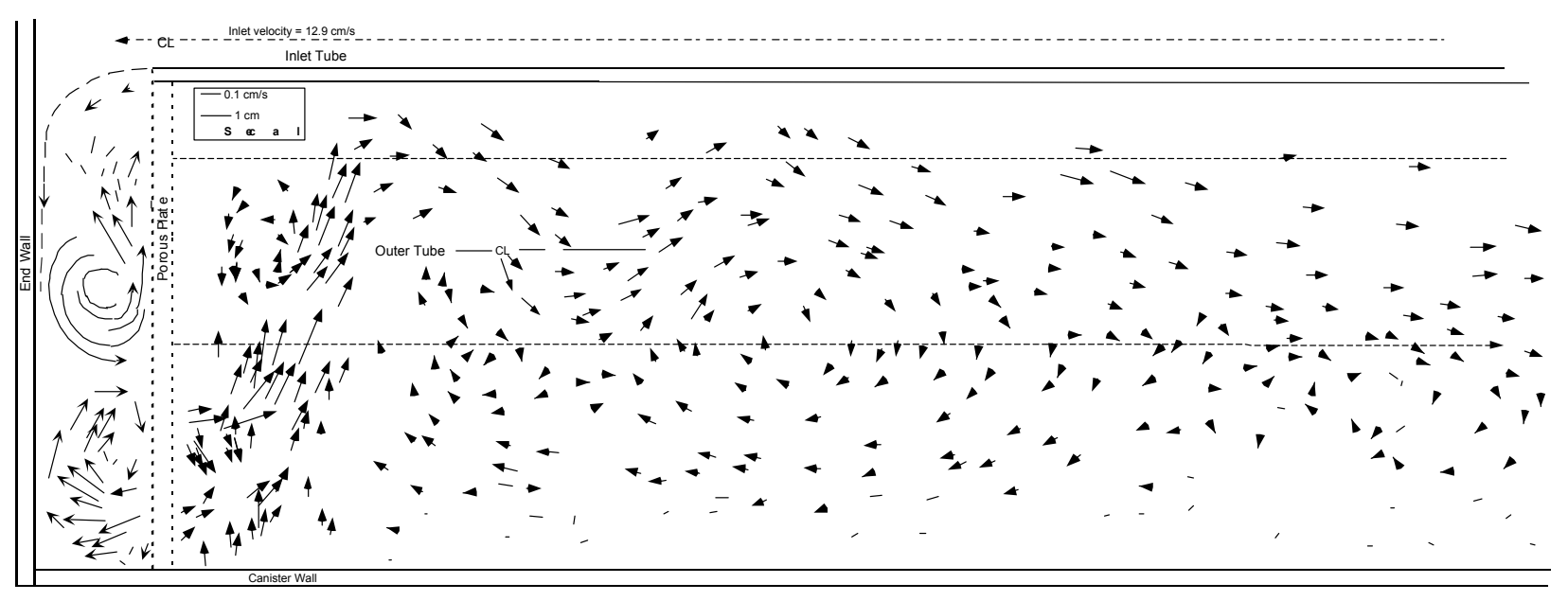

Fig. 9. "Instantaneous" velocity distribution in a plane mid-way between the simulated fuel elements, Retube $\approx 2500$.

Discussion The experiments fulfilled the objective of providing flow field information to be used for design of the experiments conducted in the Matched Index of Refraction (MIR) flow loop. A successful means of selecting neutrally buoyant particles for use in either this water flow loop or in the oil-filled MIR flow loop was also demonstrated. The experiments accentuate the difficulties of obtaining accurate velocity information in a non-index matching flow loop and of characterizing a transient three-dimensional flow field with two-dimensional short duration representations.

It is apparent from these experiments that despite the conceptual simplicity of the canister design, the flow pattern is very complex. Of concern is the uneven flow distribution near the surface of the simulated fuel rods. Higher velocity is observed near the surface towards the canister centerline than towards the outside, where, except near the perforated plate, the velocity is an order of magnitude lower than near the inside. A more even flow distribution is expected to provide more uniform drying and passivation in the radial direction. Possible design changes that could provide a more uniform distribution include (1) an optimized distribution of perforated plate holes, (2) a longer inlet plenum length (to decrease radial velocity tangent to the perforated plate which will presumably provide a more even flow distribution) and (3) a higher packing density of fuel rods, although criticality considerations may not permit this approach. The canister and plate geometry might be optimized using a CFD code once it has been verified that the code can predict the flow field reliably.

The mean flow in the semi-confined impinging jet region between the bottom of the canister and the perforated plate ("plenum" region) was not sensitive to the presence of fuel elements in the 
basket. However, in independent studies, McCreery [1999] found that the location of the vortices that form at the separation of the wall jet was sensitive to the plenum aspect ratio, $\mathrm{s} / \mathrm{D}$. This trend is comparable to that found by Morris and Garimella [1996] for confined impinging jets. For both the semi-confined (present) and confined cases, the non-dimensional location varies approximately as the square root of the aspect ratio. An additional influence is to provide a more even distribution of flow through the plate as aspect ratio increases. The most evenly distributed flow (although far from perfect) was found for an aspect ratio of four. This result may be due to the occurrence of decreasing radial velocity and a more even pressure distribution adjacent to the plate as aspect ratio increases.

\section{INEEL Matched-Index-of-Refraction flow system}

INEEL has the world's largest Matched-Index-of-Refraction (MIR) flow system; matching of the refractive indices of models and fluid allows measurements in and around complex geometries by optical techniques without disturbing the flow. A 0.6 -scale model has been installed in this unique facility to obtain detailed pointwise data for the three-dimensional turbulence field (and mean velocity field) by laser Doppler velocimetry (LDV). New velocity and turbulence measurements to date have concentrated on

- low-Reynolds-number turbulent flow in the entry tube and

- the semi-enclosed impinging jet outflow or "plenum" region

for a tube/jet Reynolds number of 2510. These measurements examine the first two flow phenomena in the sequence which leads to surface passivation and/or drying (Figure 2).

The benefit of the MIR technique is that it permits optical measurements to determine flow characteristics in passages and around objects to be obtained without locating a disturbing transducer in the flow field and without distortion of the optical paths. With a transparent model of different refractive index than the working fluid, the optical rays can be refracted in such a manner that measurements are either impossible (e.g., cannot "see" the desired location) or require extensive, difficult calibrations. Thompson, Bouchery and Lowney [1995] demonstrated this situation conceptually when laser Doppler velocimetry is applied to a rod bundle; with refractive-indexmatching the measurement and position determination are relatively straight forward while without matching the beams may not cross to form the measurement control volume at the desired focal length, if they cross at all. The MIR technique is not new itself; Corino and Brodkey [1969] employed it to measure turbulence structure in a circular tube earlier. Recent applications of the technique include, but are not limited to, those of Durst, Jovanovic and Sender [1993], Parker and Merati [1996], Cui and Adrian [1997] and Becker et al. [1999, 2002]. 
The innovative advantage of the INEEL system is its large size, leading to improved spatial and temporal resolution compared to others. To date most other experiments with index matching have been small, with characteristic lengths of the order of five $\mathrm{cm}$ or less. In contrast, the INEEL MIR flow test section has a cross section of about $60 \mathrm{~cm} \mathrm{x} 60 \mathrm{~cm}$ and is about two meters long, allowing the use of models of substantial size. Since the system volume is over 3000 gallons, a light mineral oil ("baby oil without perfume") was selected as the working fluid due to environmental and safety considerations; its refractive index matches that of some quartz. Figure 10 shows the test section with a test model installed for the present study. With the fluid temperature controlled, the quartz model can barely be seen at some wave lengths in the visible spectrum, e.g., the plates aligned vertically near the intersecting laser beams; indices are matched for the blue and green beams of an argon-ion laser (the external end containment plate is easily seen since it is made of acrylic plastic). The design flow rate can give Reynolds numbers up to about $10^{5}$ based on the cross section of the test section. The refractive index of the fluid is maintained at the desired value by a parallel temperature control system which maintains a constant temperature in the test section to within 0.1 C. In measurements in an experiment on transition induced by a square rib, meaningful velocity and turbulence data were obtained as close to the surface as $\mathrm{y}^{+} \approx 0.1$ and less [Becker et al., 1999].

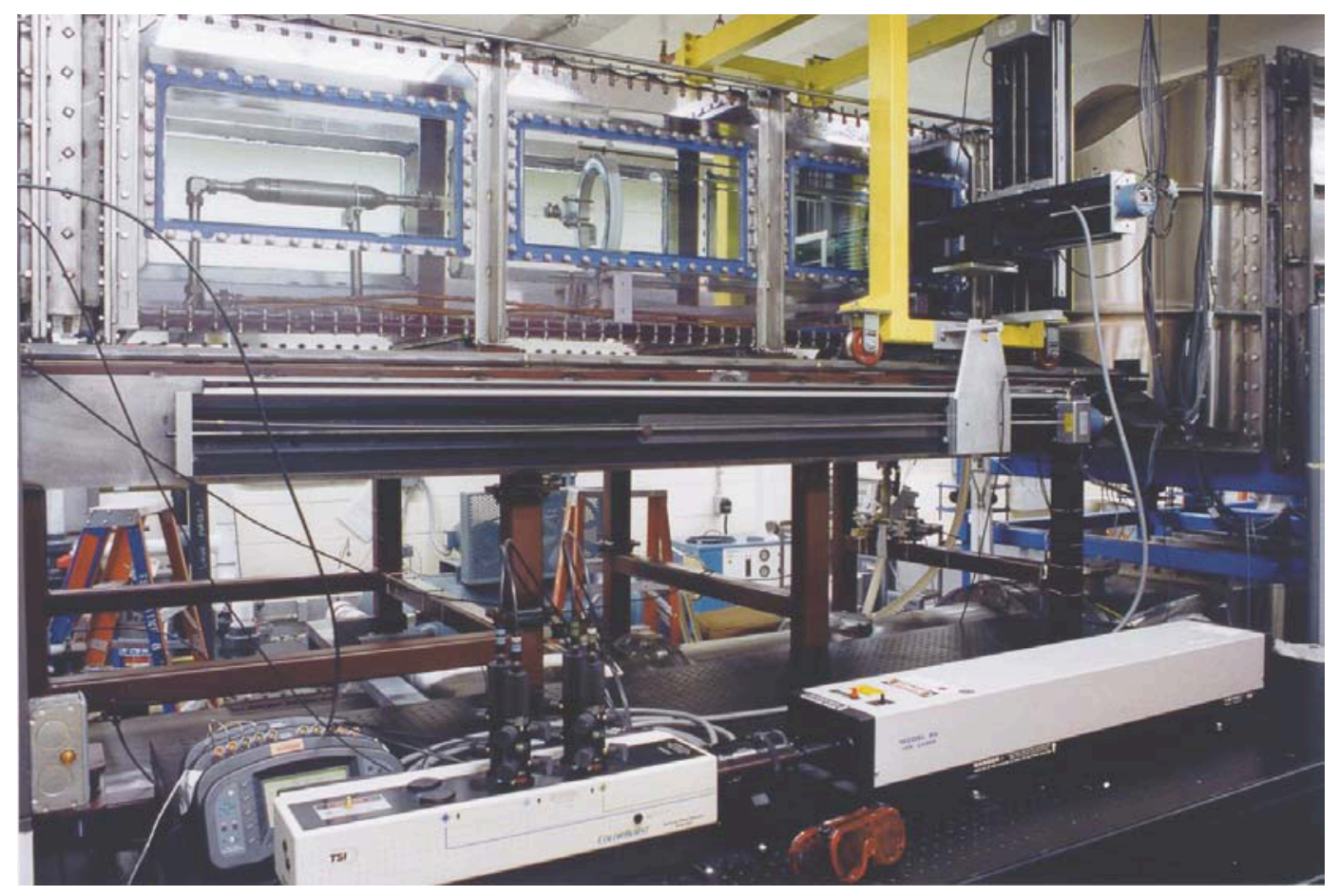

Fig. 10. INEEL Matched-Index-of-Refraction flow system with quartz model installed to study the fluid physics of an idealized SNF storage canister (present study). Also seen are the LDV transmitting optics and three-directional traversing mechanism. 
Budwig and his students measured the viscosity, density and refractive index of our mineral oil as a function of temperature in his laboratory at the University of Idaho [Orr, Thomson and Budwig, 1997]. The thermal conductivity of the preliminary sample of the oil was measured by Sparell [1995] at three temperatures using the test method of ASTM E1225, modified for liquids. Based on the specific gravity one may estimate the specific heat from handbook relations. From this information, one may estimate the Prandtl number $(\operatorname{Pr}=v / \alpha)$ to be about 250 at $24^{\circ} \mathrm{C}$.

The main loop and temperature-control loop are equipped with several thermistor probes for temperature measurements. Differential pressure transducers and manometers can measure flow speed and static pressure using several pitot-static probes and various wall static pressure taps. An available hot-film anemometer system, for velocity or wall shear stress data, consists of a Dantec 56B10 Main Frame unit equipped with a 56N22 Mean Value unit and seven 56C17 constant-temperature bridges. The output of the constant temperature bridges can be acquired using a Power MacIntosh personal computer and its National Instruments MIO board. Available LabView software can display and store mean velocities, rms velocities and spectra information.

\section{Velocity and turbulence measurements are primarily obtained with a two-component,} TSI fiberoptic-based laser Doppler velocimeter. Transmitting (and receiving) optics are provided by a Model 9832 two-component fiber optic probe for 514.5 and $488 \mathrm{~nm}$ with a lens of $350 \mathrm{~mm}$ focal distance. Included in the probe is a Model 9832-XX-Lens collimating lens option to reduce the measuring control volume; the diameter is estimated to be about $60 \mu \mathrm{m}$ in the MIR mineral oil. The laser is a water-cooled, 4 watt argon-ion Model Lexel 95. Beam splitting and frequency shifting are provided by a Model CB-2F "ColorBurst" system. Signal analysis and data processing are accomplished via a Model S65-2 two component signal analysis system including a two-channel IFA 655 digital burst correlator and FIND-W (Flow INformation Display -Windows-based) software. For acquiring additional signals such as flow rate, temperature and hot-film measurements simultaneously, a TSI Model DL4 four-channel "Datalink" multichannel interface is available.

Data acquisition is controlled via the FIND-W software for the LDV system. Desired traversing locations plus measuring parameters are specified by the operator and then the sequence is initiated. Data reduction to determine mean quantities and/or spectra is accomplished later, using other FIND$\mathrm{W}$ subprograms. Ultimately, the raw time series data are transferred to permanent magnetic or optical storage media such as compact discs (CDs). 
While the backscattering LDV mode [Durst, Melling and Whitelaw, 1976] is more convenient in operation, the validated data rate is much lower than when using forward scattering for the same conditions. To obtain statistically meaningful results, longer measuring times are required with backscatter than forward scatter. Since its low velocities lead to inherently "slow" characteristic times for flow phenomena in the MIR system, it is desirable to employ forward scattering to avoid longer measuring times than needed. The traversing mechanism was designed to use the LDV in the forward scattering mode and to avoid relative motion between the test section and the optics. To accomplish the latter objective, it is mounted directly on the structure supporting the test section. For vertical motion, platforms on each side of the test section are moved simultaneously under precise computer control to maintain alignment of the transmitting and receiving optics.

In order to match the refractive indices, the refractive index of the oil is varied by adjusting the oil temperature via the temperature control system. The temperature at which the indices are matched best was determined by experiment. With the LDV placed so one blue beam passed through a quartz rib and one green beam passed through a horizontal plate of a test model, the LDV data acquisition was operated in the two-velocity coincidence mode and oil temperature was gradually varied [Stoots et al., 2001]. The maximum sampling rate that was obtained served to indicate the best temperature for operation so that the blue and green measuring control volumes were "most coincident" (the refractive indices vary with wave length so there would be a slight difference in positions when in oil). For the oil in the MIR flow system this maximum occurred at about $23.7^{\circ} \mathrm{C}$.

Experimental uncertainties The following uncertainty analysis is derived largely following the approach of MacManus et al. [1996]. Instead of presenting a single overall uncertainty value, individual sources of uncertainty are discussed.

Particle Behavior. LDV measurements require seeding of the flow field with a second phase such as particles, bubbles or droplets. In this work, it is believed microbubbles were the seeding particles. There are two criteria that must be met to ensure that the movement of the bubbles truly represents the turbulent movement of the continuous phase. First, the size of the bubbles must be smaller than the Kolmogorov microscale. Second, the inertial relaxation time of the bubbles should be less than the Kolmogorov time scale. The procedure recommended by Calabrese and Middleman [1979] may be used to estimate the inertial relaxation time [Stoots et al., 2001]. 
Frequency Measurement. The manufacturer's specifications claim that their IFA 655 digital burst correlator has a resolution of 0.05 per cent of the reading.

Optical Errors. Great care has been taken by the investigators to ensure that the alignment and intersection of the beams within the flow field are correct. Since the index of refraction of the oil is wavelength-dependent as well as temperature-dependent, matching indices of refraction for both beam wavelengths simultaneously is impossible; the optimum temperature for the green component of laser light is not exactly the optimum for the blue. Thus, some misalignment can be expected when more than one beam passes through quartz and oil. The temperature at which the indices are matched best for both components was ultimately determined experimentally. In order to match the refractive indices, the refractive index of the oil was varied by adjusting the oil temperature via the temperature control system. In an earlier experiment, with the LDV placed so one blue beam passed through a quartz rib and one green beam passed through a quartz horizontal plate of test model, the LDV data acquisition was operated in the two velocity coincidence mode for short time series and the oil temperature was gradually varied [Stoots et al., 2001]. The maximum sampling rate that was obtained served to indicate the best temperature for operation so that the blue and green measuring control volumes are most coincident. For the oil in the MIR flow system this maximum in coincidence sampling rate occurred at about $23.7^{\circ} \mathrm{C}$. A comparable test conducted with the small central tube of the present model reconfirmed this temperature as best for its GE 214 quartz. At this condition the reduction in overlap of the two MCVs is estimated to be about thirty per cent of their lengths, thereby reducing the size of the effective MCV when operating with the coincidence mode. There is also an uncertainty in the crossing angles of the beams (and focal distances) in air or oil that leads to uncertainties in the velocities [Schwartz, Lesniak and Murthy, 1999]. The value is an approximately constant error but usually our normalized presentation will cause this error to cancel from the results presented.

Statistical Uncertainties Due to Finite Sample Size. Estimation of the statistical errors encountered due to a finite sample size followed the approach presented by Bendat and Piersol [1986] and used a typical sample size of 10,000 and measurement time of six to eight minutes. Yanta and Smith [1973] have plotted the number of points required to obtain desired accuracies in the statistical estimation of mean velocity and its standard deviation (rms fluctuation) within a given confidence level. They conclude that with $\mathrm{u}^{\prime} / \mathrm{U}$ of about fifteen per cent, 1000 data points would provide uncertainties of about one per cent in the mean velocity with a $95 \%$ confidence level. For $\mathrm{u}^{\prime}$ they predict that 1000 data points would give a statistical uncertainty within about five per cent with a $95 \%$ confidence level. These estimates likely do not take into account the 
duration required to obtain the specified number of independent samples [Becker, 2001]. Further, in some low velocity regions considerable fewer samples were obtained at individual locations.

Positional Accuracy. For all three directions, motion is accomplished with traversing mechanisms consisting of a spindle and a stepper motor. The spindle advance is 40 threads/inch. The stepper motor gives 200 steps per revolution, corresponding to an advance of about $3 \mu \mathrm{m}$ per step. The position is determined with an "Accu-Rite" indicator with manufacturer's specifications of an accuracy of $+/-0.0001$ inch or about $2.5 \mu \mathrm{m}$; its meter reads in increments of $2 \mu \mathrm{m}$. Indicated variation in position while the system is warming is about $5 \mu \mathrm{m}$. The positional accuracy becomes important in deducing the wall shear stress and the wall location from fitting the measurements for $y^{+}<\sim 3$. Thus, the absolute uncertainty of a position is of the order of $5 \mu \mathrm{m}$ but for positioning during a continuous traverse the relative positioning uncertainty is less.

Temperature Accuracy. The index of refraction for the oil is a relatively strong function of temperature [Orr, Thomson and Budwig, 1997]. Mismatch of the indices of refraction for the oil and quartz plate can result in movement of the measurement volume as well as distortion of the fringes within the measurement volume. Durst, Keck and Kline [1979] recommended that the indices of refraction should be matched to the fourth decimal point (i.e., 0.0001). For the oil used in this work, the temperature control must therefore be better than $+/-0.3^{\circ} \mathrm{C}$. Temperature control tests were conducted with both water and oil in the loop. Measurements of the spatial and temporal variation with a precision thermistor probe have shown spanwise uniformity and steadiness within $0.1^{\circ} \mathrm{C}$ [Stoots et al., 2001].

Uncertainties in oil properties. The properties of the light mineral oil were measured by Orr, Thomson and Budwig [1997]. An indication of the precision of these data is provided by their variations for $3 \sigma$; these were $0.002 \mathrm{~g} / \mathrm{cm}^{3}$ and 0.3 centistokes or about 0.3 and two per cent of typical values, respectively. The latter uncertainty would dominate the uncertainties in Reynolds numbers, making them of the order of two per cent of their calculated values.

Calibration. To obtain a measure of the accuracy of the overall LDV system during its initial installation, the mean velocity indicated by the data processing system was compared to that of a rotating "calibration wheel" passing through the measuring volume formed by the laser beams. The indicated velocity agreed with this standard to within about 0.3 per cent. The 
variation along the axis of the measuring volume was less than one per cent of its average value; this variation is a contributor to the apparent turbulence intensities of the LDV technique [Condie et al., 1998].

\section{Turbulence and velocity distributions by laser Doppler velocimetry}

For the turbulent fluid physics experiments, a circular quartz model was designed, fabricated and installed horizontally. The design is described in Figure 11. With the exception of the bolts providing axial constraint, the materials are transparent; the structural end plates are acrylic and the remaining sections are finished quartz tubes and plates to match the refractive index of the light mineral oil. Tubular sections were fabricated from GE 214 quartz while flat ones were of GE 124 quartz.

The central tube has an internal diameter $\mathrm{D}$ of $14.99 \mathrm{~mm}$ and a length of about $105 \mathrm{~cm}(\mathrm{~L} / \mathrm{D} \approx$ 70). In the present configuration the nominal dimensions are "plenum" spacing $H / D=s / D=2$, canister internal radius $\mathrm{r}_{\mathrm{O}} / \mathrm{D}=9$, pitch of holes in the perforated plate $\mathrm{ph} / \mathrm{D}=1$ and hole diameter $\mathrm{d}_{\mathrm{h}} / \mathrm{D}=1 / 4$ in a square pattern. The configuration and dimensions of the perforated "bottom" support plate are presented in Figure 12. Quartz tubes representing spent fuel elements may be installed in the $71 \mathrm{~cm}$ long space corresponding to a basket. These dimensions are about 0.6 times those of the reference idealization. Figure 13 shows the model installed in the MIR test section with oil temperature approximately at the level for matching of the refractive indices. Since the matching is aimed at the blue and green wave lengths one can see some components faintly in other regions of the visible spectrum; also visible are some rough surfaces, such as the holes in the perforated tube.

The inlet configuration is evident in Figure 10 on the left. A small circular plenum contains a flow straightener to avoid swirl from the upstream plumbing. The flow then passes through a standard reducer and extension to a flexible tube of one inch $(25 \mathrm{~mm})$ inside diameter and approximate length of $18 \mathrm{in}$. $(460 \mathrm{~mm})$ before an abrupt geometrical contraction into the quartz central tube of the model (i.d. $=14.99 \mathrm{~mm}$ and $\mathrm{L} / \mathrm{D} \approx 70$ ). The oil was circulated to the model through an auxiliary flow loop by a centrifugal pump driven by a motor controlled by a variable frequency drive. Flow was measured with a Micro Motion Coriolis mass flowmeter, Model CMF 100. The time-averaged mass flow rate was typically held constant to within about 0.05 per cent from run to run and its rms fluctuation during a run was of the order of 0.4 per cent. 


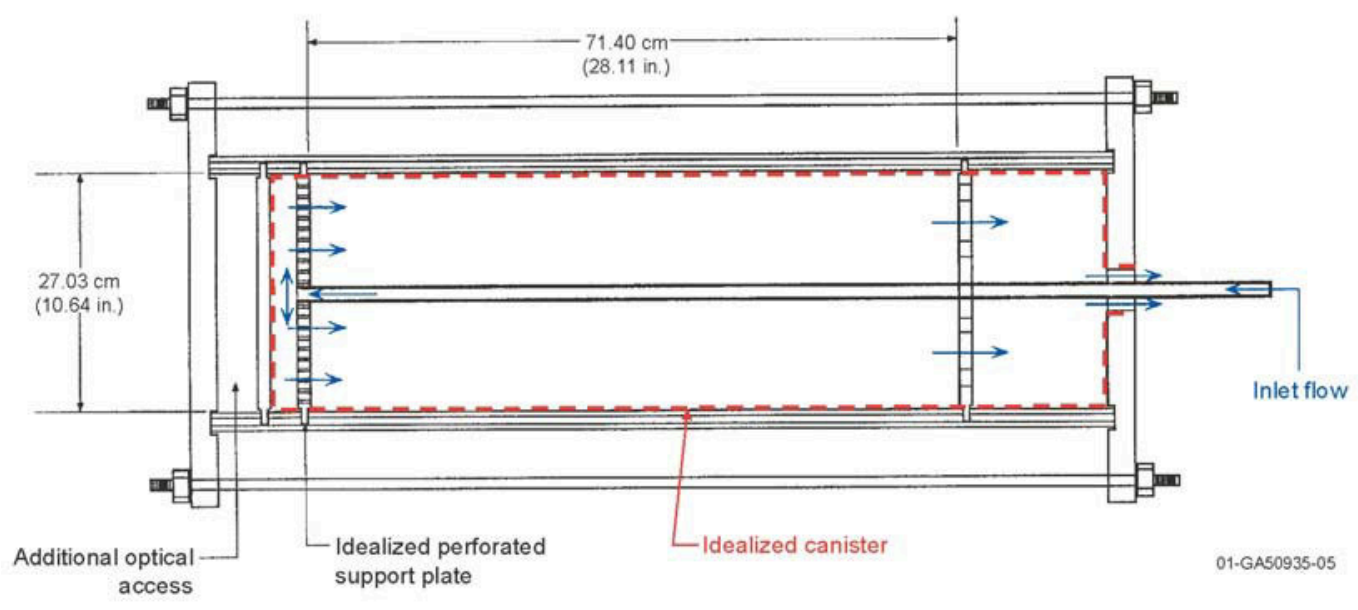

Fig. 11 Design of 0.6-scale model for studies of the generic turbulent flow processes of idealized SNF canisters.

In accordance with earlier Figure 2 presenting the fundamental fluid physics questions involved, the objectives of the LDV experiments are to determine

a) the inflow turbulence of the impinging jet and

b) the turbulence and velocity fields of the flow approaching the perforated plate

(i.e., the first two questions sequentially). The examination of these questions requires fundamental measurements of the decay of turbulence and velocity in the low-Reynolds-number impinging jet and its surroundings and of the low-Reynolds-number turbulent flow in the inlet tube.

For the present applications, design flow rates are expected to give tube Reynolds numbers between 2500 and 5000. Tabulated LDV data for mean and rms axial velocities are available from Lekakis for pipe flow at $\mathrm{Re} \approx 4130$ [Durst et al., 1996] and Satake et al. [2000] have direct numerical simulations at $\operatorname{Re} \approx 4300$, corresponding to the inlet conditions of Shehata and McEligot [1998]. Otherwise, the only profile data known in the range of interest are the tabulated mean velocity profiles by H. C. Reynolds with an impact tube at $\mathrm{Re} \approx 3020$ and 4080 [Reynolds, Davenport and McEligot, 1968] and the figures of Patel and Head [1969] at $\operatorname{Re} \approx 2440,2615,2680$, 3000,4060 and 4430 with a pitot tube. Consequently, a byproduct of the present measurements is a set of new data for Reynolds stresses and mean velocities for flow in the range $2500<\operatorname{Re}<4000$ (along with laminar measurements for comparison to the analytical prediction, e.g., Kays [1966]). 


\section{LOWER TUBE SUPPORT PLATE}

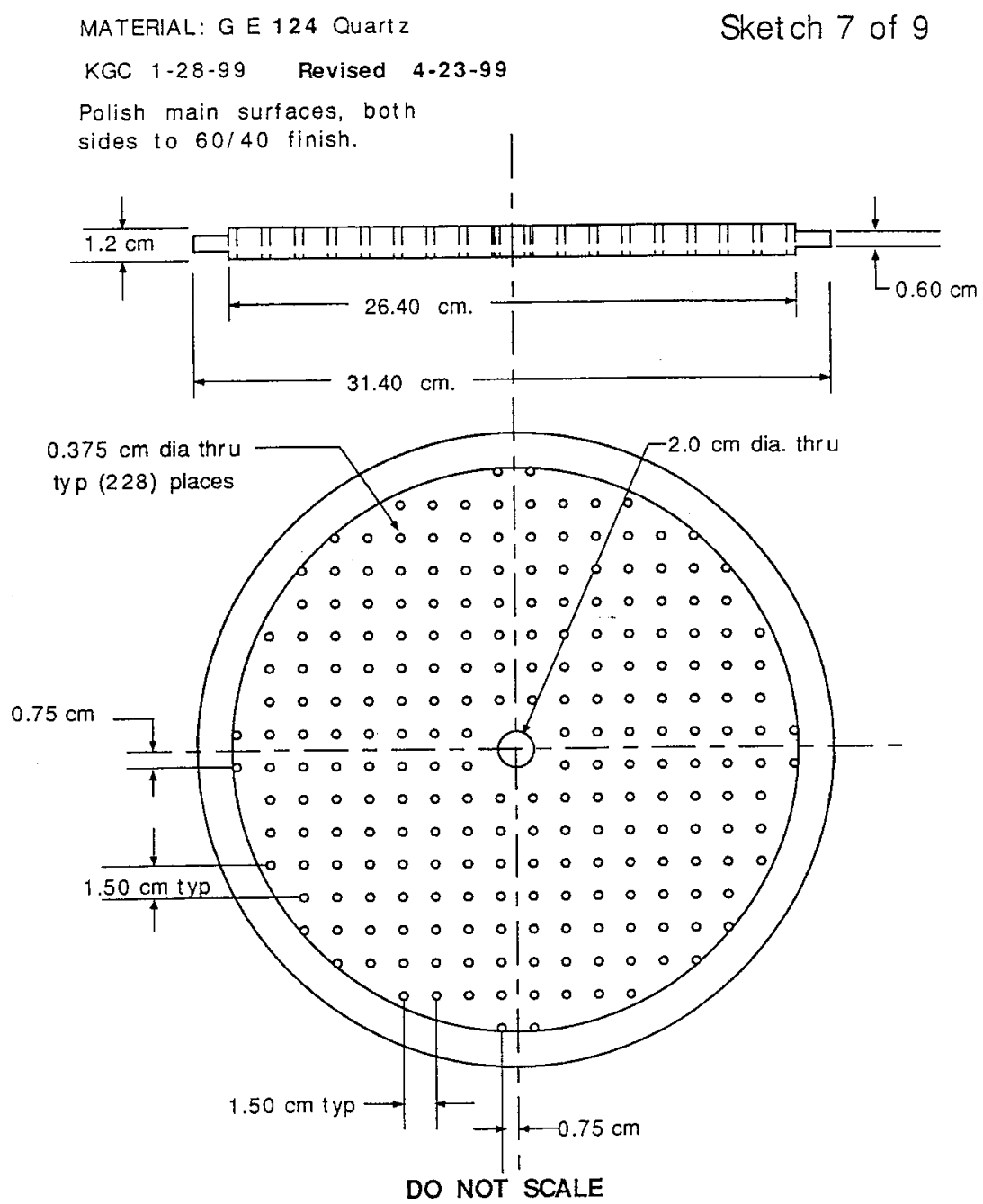

Fig. 12. Fabrication sketch for simulated basket support plate.

As noted, most of the present data were obtained at $\mathrm{Re} \approx 2500$. At this Reynolds number for a tube of the present L/D of about 70, the velocity field can be expected to be fully laminar, fully turbulent or an intermittent flow undergoing a transition from the former to the latter [Rotta, 1956; Patel and Head, 1969]. For the development of a laminar flow from a uniform inlet velocity, Kays [1966] suggests that the approach of the friction factor towards $16 / \mathrm{Re}$ is a reasonable measure of the development of the velocity profile. Based on the analysis of Langhaar [1942], he notes that the friction factor is within two per cent by about $(\mathrm{x} / \mathrm{D})=\mathrm{Re} / 20$ which converts to $\mathrm{x}^{+}=\mathrm{x} /\left(\mathrm{r}_{\mathrm{W}} \mathrm{Re}\right) \approx 0.1$ in terms of laminar entry coordinates. For a Reynolds number of 1500, this distance is about 75 diameters and at $\mathrm{Re} \approx 2500$ it would be about 125 diameters. 
Initial measurements of $\mathrm{U}, \mathrm{V}, \mathrm{u}^{\prime}, \mathrm{v}^{\prime}$ and $\overline{\mathrm{u}} \mathrm{v}$ have been obtained in the tube, about 1.7 diameters upstream from the exit for $\operatorname{Re} \approx 1500,2510$ and 3500. These data provide the initial conditions for the jet which impinges on the bottom of the simulated canister. They also permit assessment of the models employed in numerical predictions.

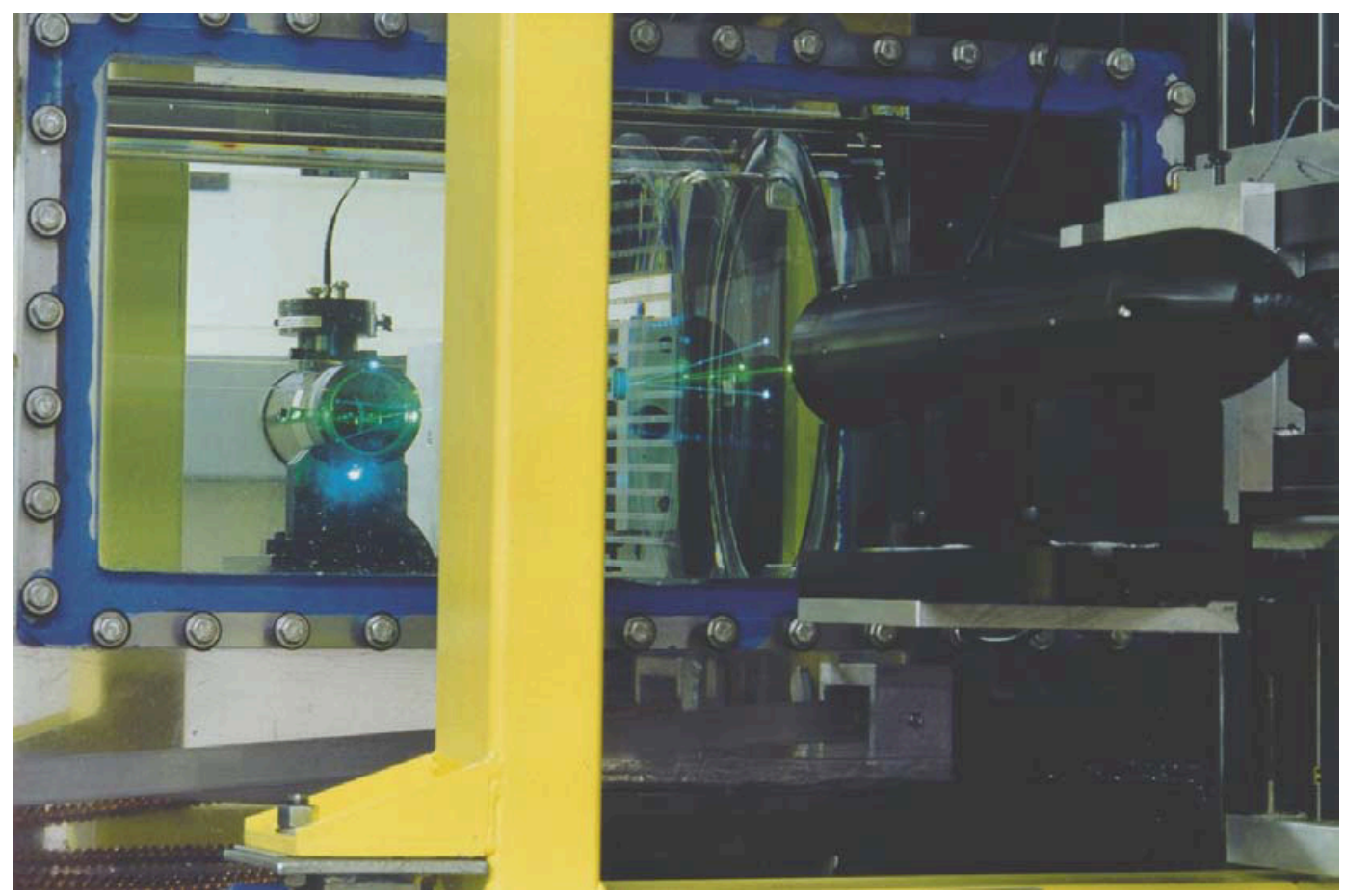

Fig. 13. Model installed in MIR test section; black ring is a gasket sealing the acrylic end plate.

At $\mathrm{Re} \approx 1500$ the mean axial velocity profile $U\{r\}$ agrees closely with the expected parabolic profile for a fully-developed flow in a tube. At $R e=2510$ this profile is flat across the central region of the tube as shown in Figure 14 (the data from the tube are represented by the diamond symbols). For a fully-developed laminar flow, the centerline velocity would be twice the bulk velocity whereas for fully-developed turbulent flow it would be expected to be $\mathrm{U}_{\mathrm{c}} / \mathrm{V}_{\mathrm{b}} \approx 1.3$ or so. The appearance of the profile is that of a developing one with a laminar boundary layer growing near the wall but not yet reaching the centerline. Since the measurement station is about 68 diameters from the tube entrance, this observation would be consistent with a laminar development length of about 125 diameters for this Reynolds number. However, the data for root-mean-squared fluctuations, $\mathrm{u}^{\prime}$ and $\mathrm{v}^{\prime}$, show evidence of turbulent behavior. 


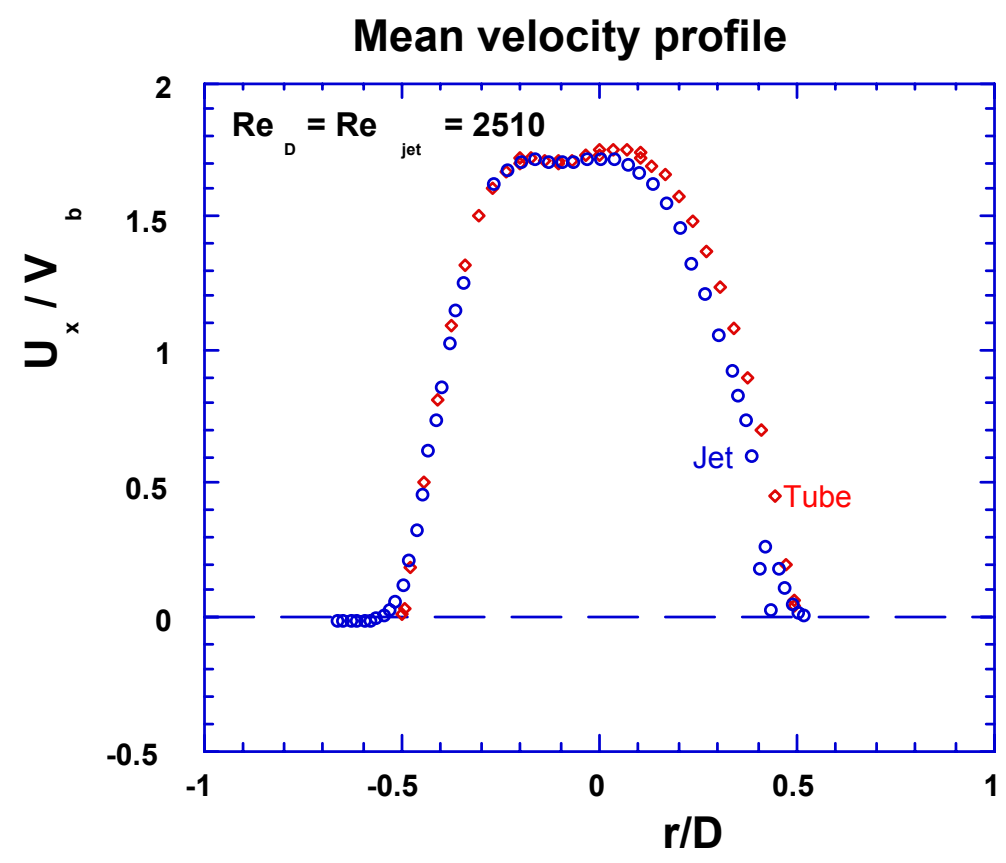

Fig. 14. Axial mean velocity distribution entering the plenum region; diamonds = in tube near exit, circles $=$ initial station of entering jet.

The data for $\operatorname{Re}=2510$ and 3510 have been examined in terms of the wall coordinates employed normally for developed turbulent flows. The variable y is the distance from the surface. Since the high-Reynolds-number asymptote is approximately logarithmic $\left(\mathrm{u}^{+} \approx 5+2.5 \ln \mathrm{y}^{+}\right)$, semilogarithmic coordinates are employed [Kays and Crawford, 1980]. Results for the mean velocity are compared in Figure 15 to each other, to other investigators, to a laminar asymptote and to the turbulent "law of the wall." For fully-developed, low-Reynolds-number turbulent flow the levels of the profiles decrease as the Reynolds number is increased until they approach the "law of the wall" or "universal" velocity profile approximately [McEligot, Ormand and Perkins, 1966]. The data for $\operatorname{Re}=2510$ have the appearance of a Blasius (laminar) boundary layer profile [Schlichting, 1979] when plotted in this form. Other investigators's measurements for the range $3000<\operatorname{Re}<4400$ are taken from the experiments of Reynolds [1969], Shehata [1984] and Durst et al. [1996]. It is seen that our values for $\mathrm{Re}=3510$ and $\mathrm{x} / \mathrm{D} \approx 68$ are consistent with the data of others and their trends, as well as the observation of Patel and Head [1969] that the transition from laminar to turbulent flow proceeds rapidly along the tube for $\operatorname{Re}>3000$. 


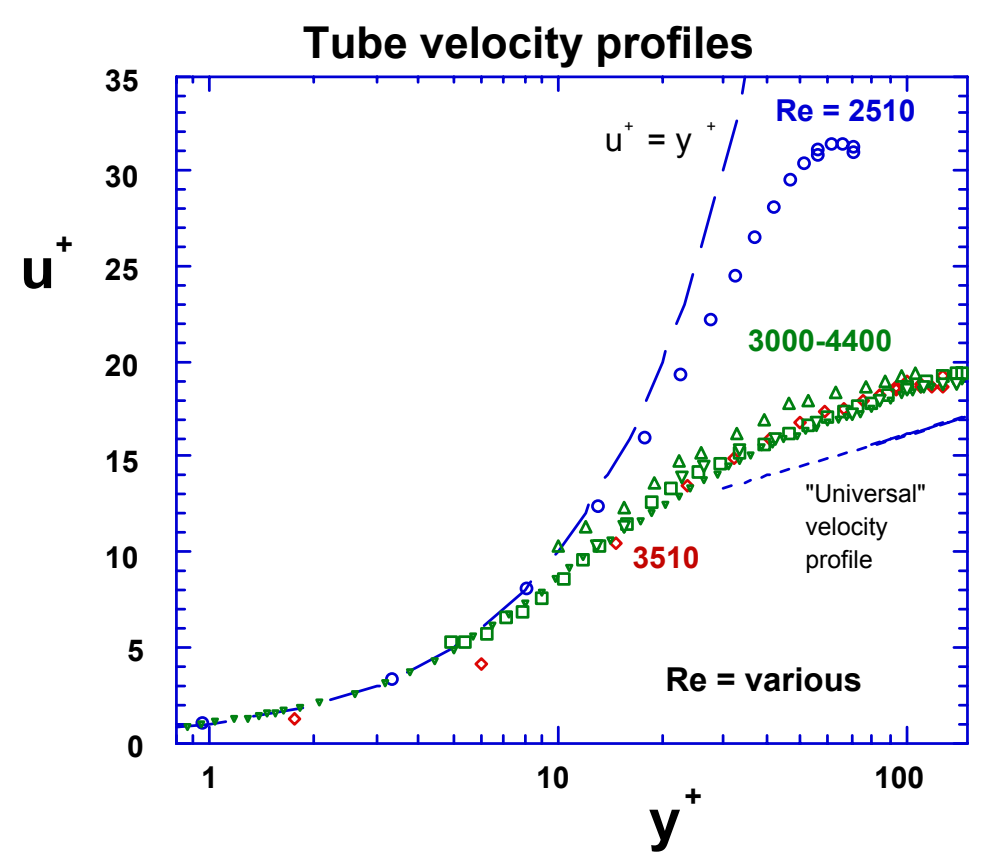

Fig. 15. Mean velocity profiles at low Reynolds numbers in circular tubes; circles and diamonds= present data.

Some preliminary turbulence measurements were examined (Figure 16). Here the rms fluctuations have also been plotted in terms of wall coordinates; the non-dimensional axial component $\left(\mathrm{u}^{\prime}\right)^{+}$is represented by diamonds and the radial ones $\left(\mathrm{v}^{\prime}\right)^{+}$by circles. These data suffer from statistical uncertainty due to low data rates or short duration of sampling but they do demonstrate significant levels of turbulence. For comparison, Figure 17 presents typical results from a number of other investigators using a variety of methods plus direct numerical simulation [McEligot and Eckelmann, 2001]. While these data are for low-Reynolds-number turbulent flow in channels, results for circular tubes have approximately the same magnitudes [Durst et al. 1996]. One sees that for fully-developed turbulent flow, $\left(\mathrm{u}^{\prime}\right)^{+}$peaks at a value of about 2.5 near $\mathrm{y}^{+}=15$ and $\left(\mathrm{v}^{\prime}\right)^{+}$has lower values with its peak further from the wall (not shown, but peak magnitudes are typically $0.8-1$ ). The present data generally have lower values at comparable locations in Figure 17.

One can conclude that the flow from the inlet tube is one that is intermittently turbulent and laminar, i.e., undergoing axial transition. However, the mean velocity profile still has predominantly laminar character. 


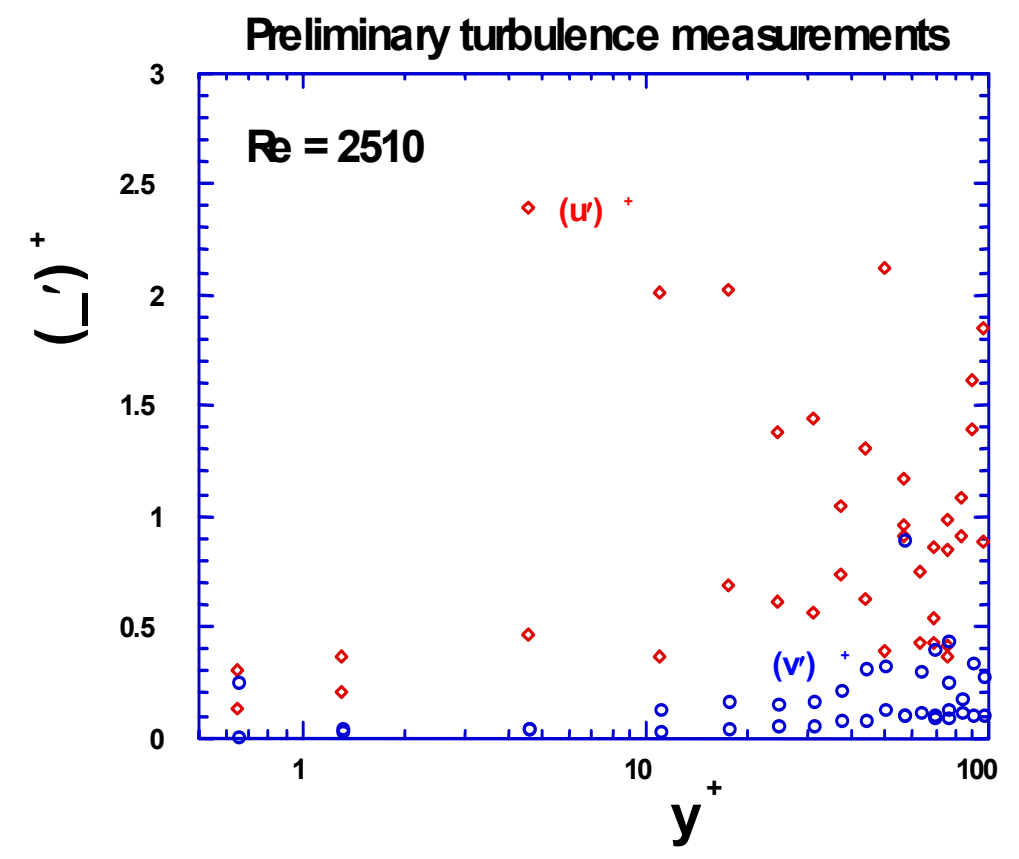

Fig. 16. Root-mean-squared velocity fluctuations near exit of central tube normalized in wall coordinates, $\operatorname{Re}=2510$.
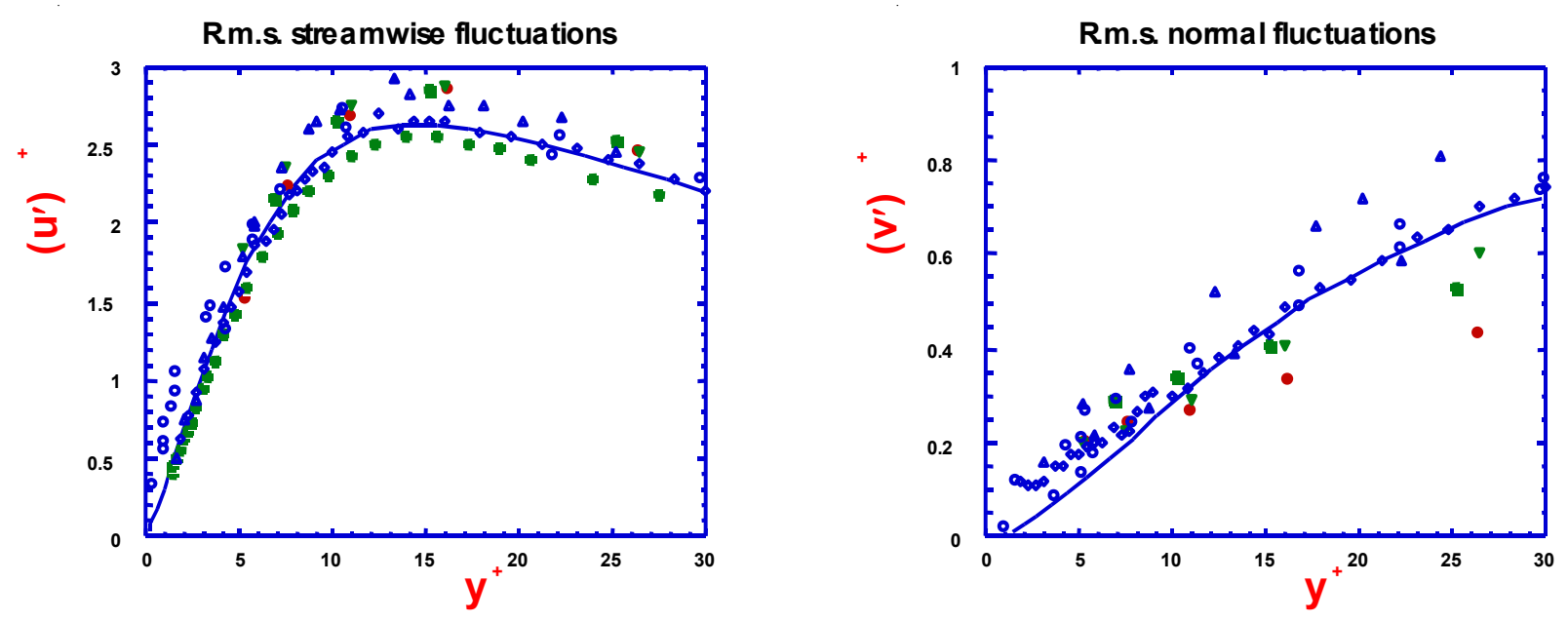

Fig. 17. Typical measurements and direct numerical simulation of root-mean-squared velocity fluctuations at low Reynolds numbers by a variety of investigators [McEligot and Eckelmann, 2001].

In the remainder of the results to be presented, most velocities and their fluctuations are normalized by the bulk velocity in the inlet tube $\left(\mathrm{V}_{\mathrm{b}}\right.$,in $)$ as one useful reference velocity. This quantity is equivalent to the bulk velocity at the jet entry and is about 55 per cent of the maximum velocity of the jet (or tube). For reporting the turbulence levels, turbulence intensities normalized by 
pointwise mean velocities are avoided because they can give misleading high values in regions of low velocities.

The initial jet profiles are shown in Figures 14 and 18; mean axial velocity is in the former and turbulence components in the latter. These measurements are at approximately 0.07 diameters from the tube exit. As expected, the mean profile is essentially the same as it is 1.7 diameters upstream inside the tube although one can see slight evidence of spreading or entrainment at the edges. For the jet, the axial fluctuations dominate over the radial ones. Highest values of $u^{\prime}$ occur in the shear layer between the flat central region and the surroundings in the plenum region and reach magnitudes that are about twenty per cent of the inlet bulk velocity; this shear layer corresponds to the upstream boundary layer in the tube. At this location the radial fluctuations are small with maximum values of the order of two per cent of $\mathrm{V}_{\mathrm{b}}$,in. Later it will be seen that in the semiconfined plenum region the radial fluctuations are much greater than the axial ones.

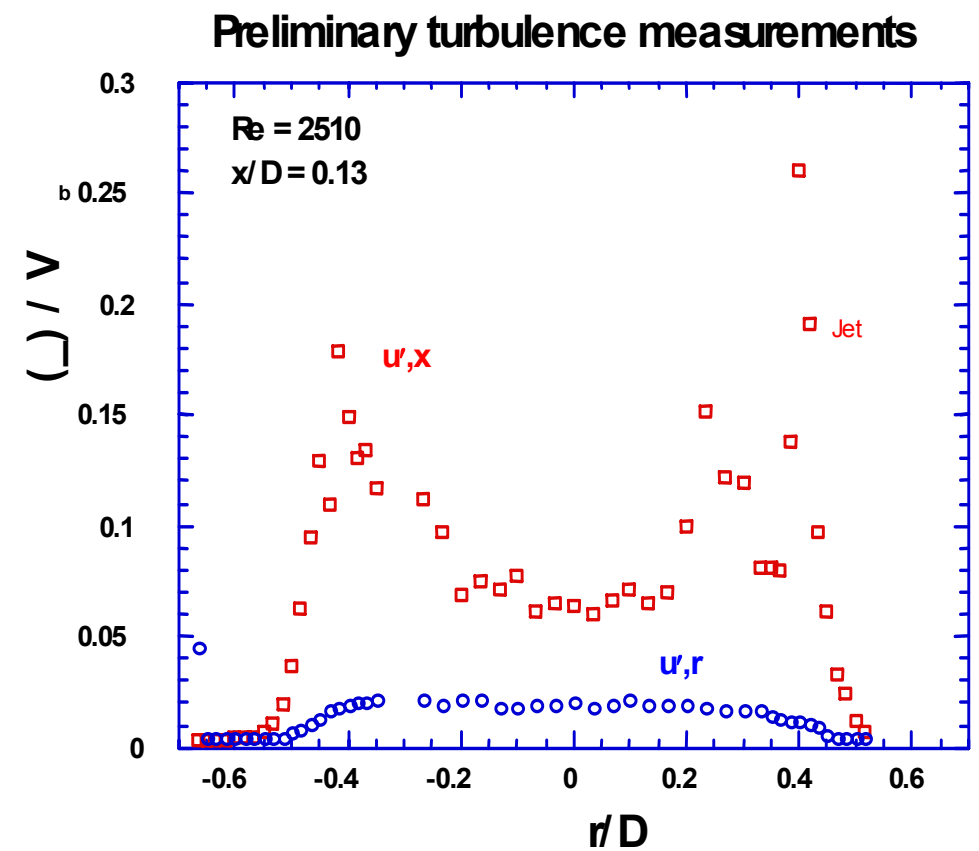

Fig. 18. Root-mean-squared velocity fluctuations of inlet jet at initial station, normalized by bulk velocity in inlet tube.

As noted under the section on Related Work, fluid physics data for impinging jets are sparse; most of the extensive literature deals with heat transfer measurements. Only a few investigators report flow measurements for confined impinging jets and apparently none have examined the semiconfined geometry of the present study. Further, only a few experiments utilized a well-developed inlet flow for the jet [Cooper et al., 1993] as in the SNF application. The present objectives include 
understanding the turbulent or laminarizing flow field approaching the perforated plate, i.e., the upstream conditions for that plate. Even for confined jets, only Fitzgerald and Garimella [1996] appear to have measured turbulence quantities outside the impinging jet and its wall jet; they present rms radial velocity fluctuations, primarily in and near the wall jet at high Reynolds numbers. Emphasis on the region outside the wall jet is lacking in their study.

Two-component LDV measurements of the turbulence structure and mean velocity distributions for the semi-confined impinging jet and its surroundings upstream of the perforated plate were conducted. Time series data have been obtained for

a) radial profiles in the semi-confined region at $0.046<\mathrm{x}^{\prime} / \mathrm{D}<1.97$,

b) "free" jet profiles at $-\mathrm{x} / \mathrm{D} \approx 0.07,0.5,1.0$ and $1.5 \quad$ plus

c) wall jet profiles at $\mathrm{r} / \mathrm{D} \approx 1$ and 1.3

where $\mathrm{x}^{\prime}$ is measured from the solid bottom plate and $\mathrm{x}$ is taken from the tube exit opposite to the direction of jet flow (however, the axial velocity $U$ is taken as positive in the direction of the jet flow). These locations are on the vertical centerplane between the two middle rows of holes in the perforated plate so they are not directly affected significantly by upstream influence from the holes; additional subtasks would be desirable to address the behavior in a plane through a line of holes for comparison. Subtasks (b) and (c) provide data for comparisons of the initial behavior predicted numerically and to the higher Reynolds number experiment of Cooper et al. [1993].

The development of the "free" jet is shown in Figure 19 in terms of its axial mean velocity profiles. The circles are for the initial profile at 0.07 diameters and the profile at $\mathrm{x} / \mathrm{D}=-1.5$ is given by squares. There is no significant change as the jet proceeds halfway across the plenum $(H / D=2)$ except a slight increase in its breadth. By a half diameter from the impinging plate the velocity in the central region decreases as radial outflow from the stagnation region begins to feed the ensuing radial wall jet. 


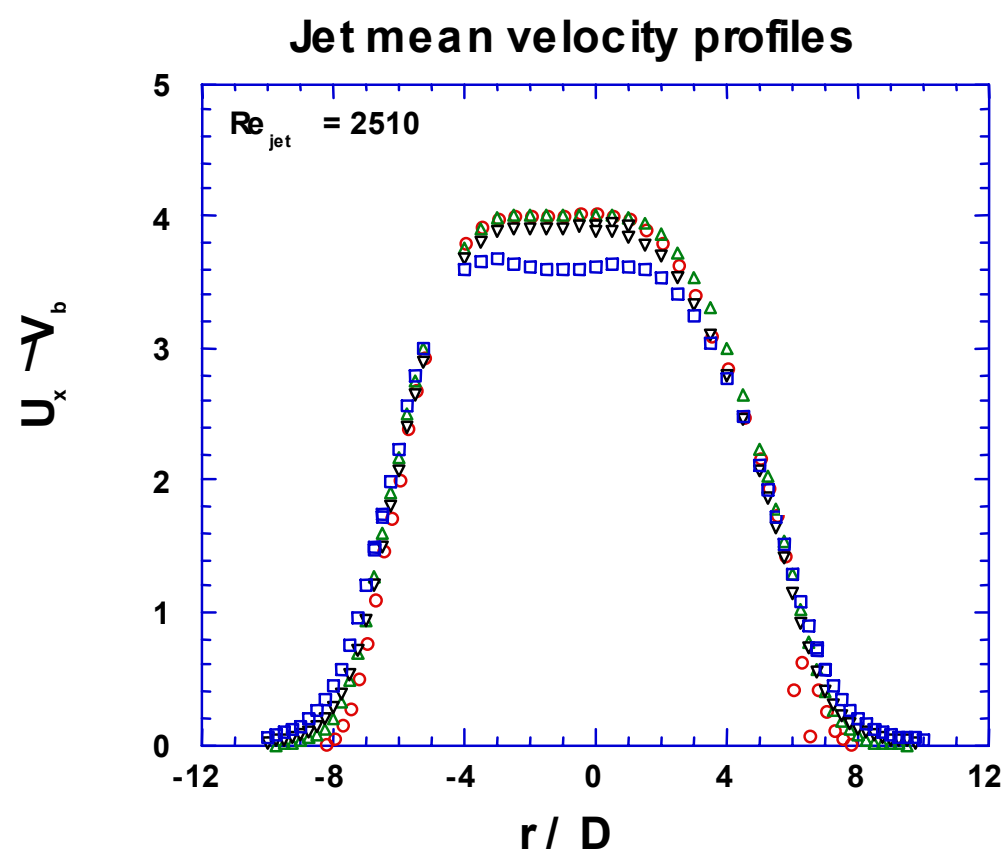

Fig. 19. Development of impinging jet from initial station to $x^{\prime} / D \approx 0.5$ from impingement plate.

Overall behavior of the radial wall jet is shown later with the plenum region results. The "initial conditions" of this jet were measured at $\mathrm{r} / \mathrm{D} \approx 1$, a location where tabulated data are also available from the experiment of Cooper et al. [1993] (courtesy of David Jackson of UMIST). Cooper et al. had an impinging jet from a fully-developed turbulent tube flow at $\operatorname{Re} \approx 23,000$, about an order-of-magnitude higher than the present experiment. Their impingement plate did not have another surface opposite to confine the flow; consequently, we refer to it as a semi-infinite impinging jet. Though there are obvious differences from the present experiment, it is one of the few experiments with time-resolved data on the flow structure of impinging jets with a welldeveloped initial flow. The spacing from the tube to the plate was the same, $s / D=2$. Available were profiles of the mean and fluctuation radial velocities at a number of radial stations. Figure 20 compares the mean velocity profiles and the turbulence measurements are in Figure 21; in these figures, the variable $y$ is the distance from the impinging plate. 


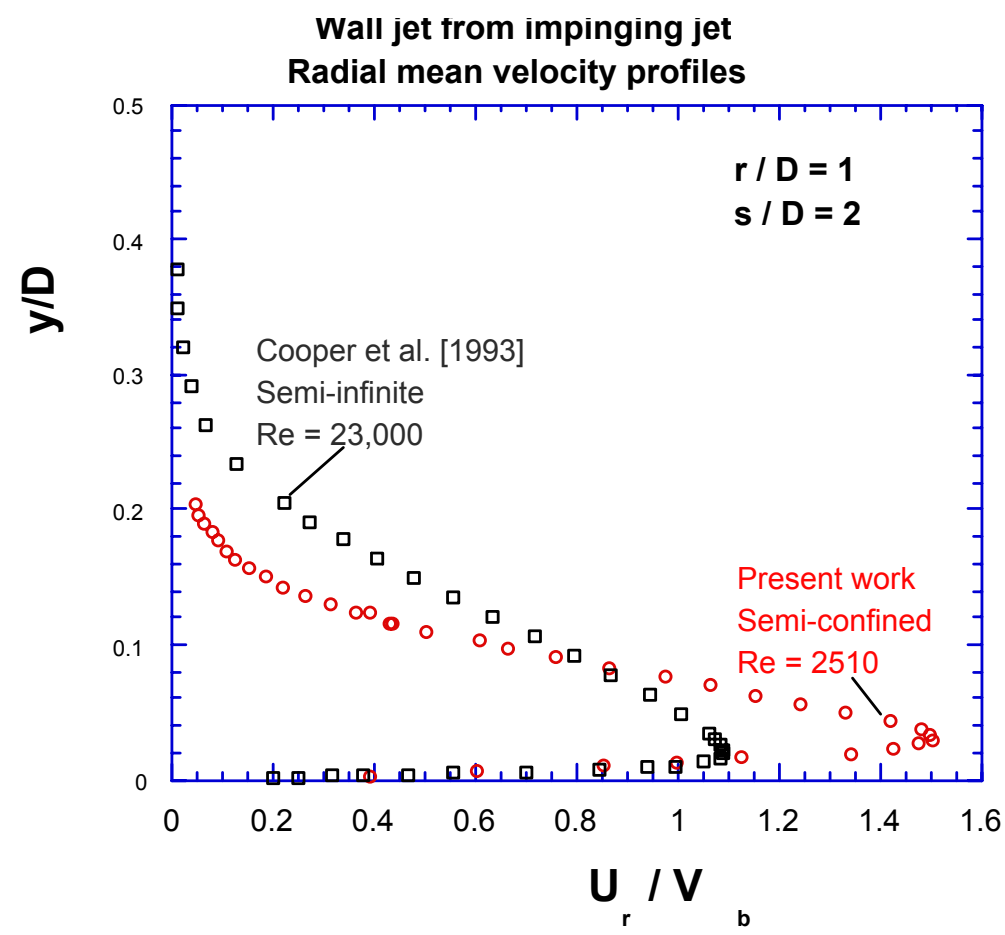

Fig. 20. Mean velocity profiles of wall jets evolving from impinging jets near impingement region, $\mathrm{r} / \mathrm{D} \approx 1$.

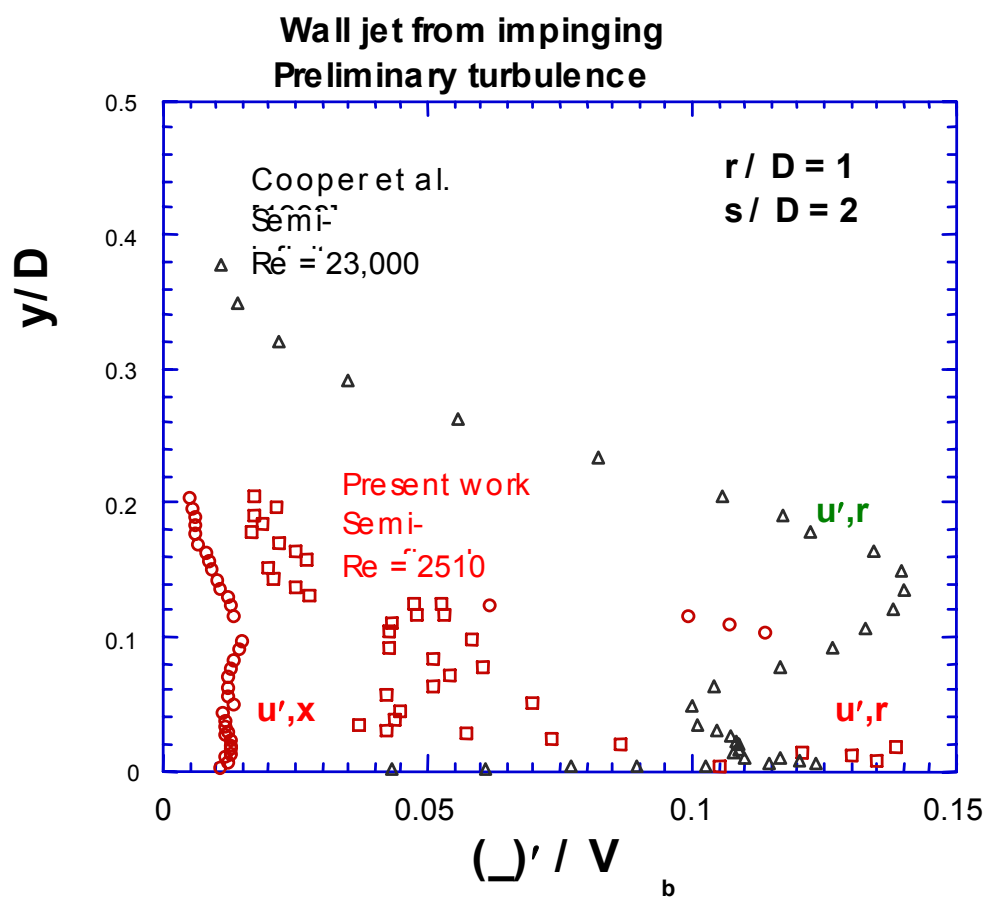

Fig. 21. Comparison of profiles of root-mean-squared radial and axial velocity fluctuations in wall jet near initiation. 
At the same non-dimensional location (one diameter from the centerline) the present wall jet is significantly thinner than the one of Cooper et al. and, consequently, the peak value of $U_{\mathrm{r}} / \mathrm{Vb}_{\mathrm{b}}$ (or $\mathrm{V} / \mathrm{V}_{\mathrm{b}}$,in) is greater. In the present experiment the maximum axial inlet velocity was about 1.8 times $\mathrm{Vb}$,in and the peak radial mean velocity was about 1.5 at this location. In the experiment by Cooper et al. the fully-developed turbulent tube flow had a lower centerline velocity, giving a lower nondimensional momentum flow rate and the resulting peak radial mean velocity was only about 1.1 at the same radial position. Despite the thicker boundary layer, the peak in their turbulent wall jet was closer to the wall than observed in the present study.

In contrast to the free jet, the radial fluctuations in the wall jet are mostly greater than the axial ones. The root cause is likely the different orientation of the nearby constraining surface. In Figure 21 the radial fluctuations $\mathrm{v}^{\prime} / \mathrm{Vb}$, in (denoted $\mathrm{u}^{\prime}, \mathrm{r}$ ) are represented by the squares and the axial ones by the circles; triangles show the radial fluctuations of Cooper et al. In both experiments significant radial fluctuations are seen further from the wall than the apparent mean boundary layer. Again the region of Cooper et al. is thicker than ours. Their fluctuation profile shows two peaks, one corresponding to the region of maximum shear stress in the outer region and the other in the nearwall region as turbulent boundary layers with an imposed freestream flow. Our v' peak is about the same magnitude as the maximum of theirs, about fourteen per cent of $\mathrm{Vb}$, in, but its location corresponds to the high shear region near the wall whereas their highest values are in the outer region. Although $v^{\prime}$ is generally greater then $u^{\prime}$ there appears to be a "spike" in the present data approximately at the high shear region at the outer edge of the wall jet (it is not clear whether this occurrence is fortuitous).

As indicated in Figure 2, the flow in the inlet plenum provides the upstream conditions for the flow through the perforated plate simulating a basket support plate. For $R e=2510$ this flow pattern is presented in Figure 22 in terms of mean velocity vectors. (This plane is on the vertical centerline of the model between two vertical rows of holes as seen in Figure 12.) Main features are the high velocity impinging jet, the thin expanding wall jet, a large recirculating eddy near the outer wall of the canister and relatively low velocities near the perforated plate. The flow in the wall jet remains attached until it impinges on the outer wall of the plenum region and induces the main recirculating eddy. Only a single major eddy is seen rather than the two observed with the water flow visualization. Outside the impinging and wall jets, flow is relatively quiescent with velocities about an order-of-magnitude less than the inlet velocity. In the plenum region upstream of the perforated plate, the radial velocity component at a point is generally greater than the axial component, except in the recirculating eddy. In the vicinity of the perforated plate, flow is primarily radially inward. 
Tabulated values of this velocity distribution can be made available for comparison with the predictions of CFD codes proposed for canister flows.

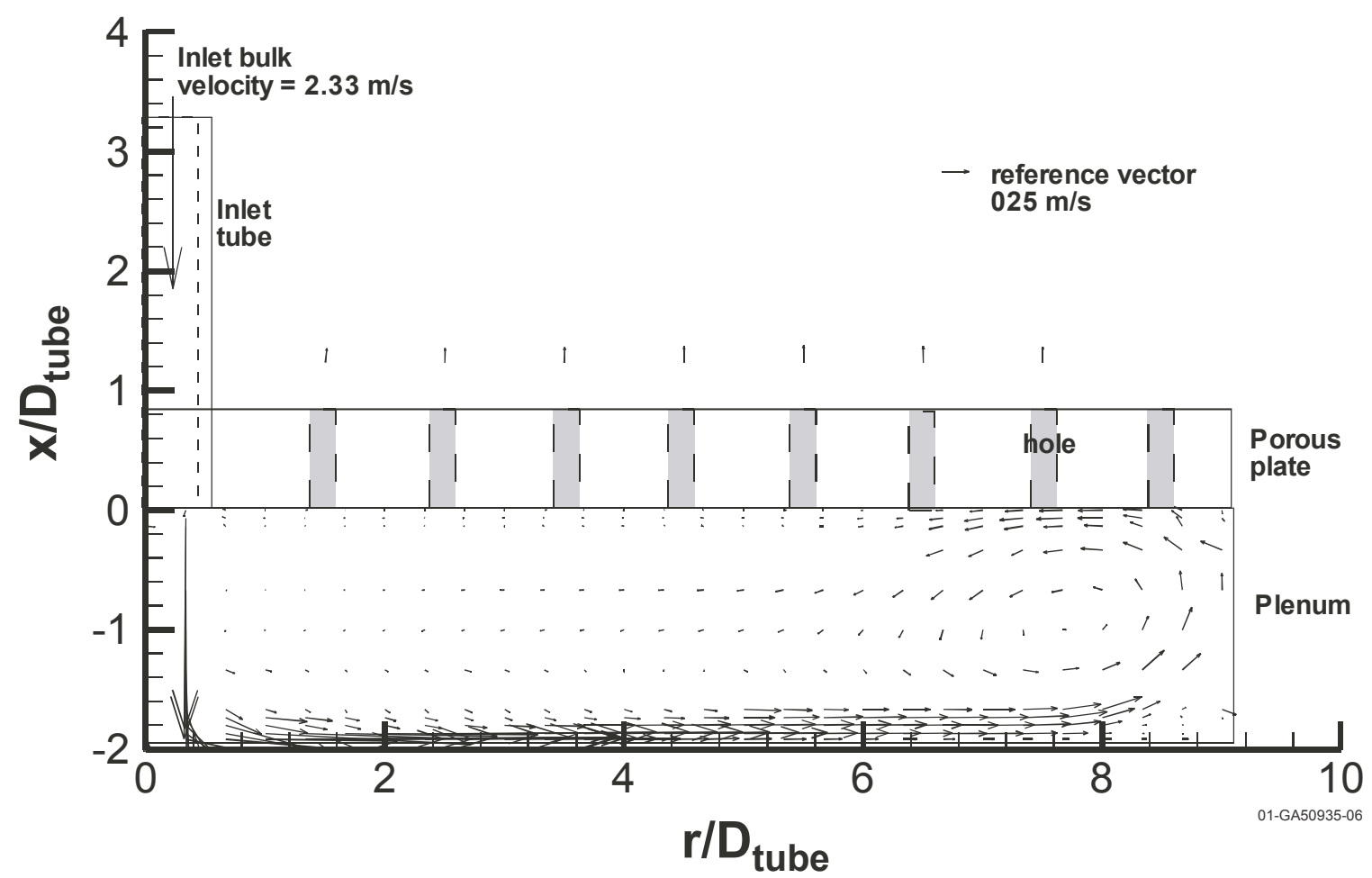

Fig. 22. Mean flow pattern of a semi-confined impinging jet and its surroundings, $R_{j}$ et $=2510$.

The turbulence field upstream of the perforated plate may be examined in terms of contour plots of the magnitudes of the root-mean-squared fluctuating velocity components. Presumably, the higher the turbulent kinetic energy $\left(\mathrm{k}=\left(\mathrm{u}^{2}+\mathrm{v}^{2}+\mathrm{w}^{2}\right) / 2\right)$ carried into the element array, the more effective the mass transfer to the elements. In the present experiment radial and axial components were determined. The circumferential component was not measured due to lack of time and funding so the magnitude of the turbulence kinetic energy is not available (by traversing along horizontal planes at equivalent positions this additional component could be obtained). The present data would be suitable for comparison to predictions from Reynolds stress models [Hanjalic, 1999] or k- $\varepsilon-\mathrm{v}^{2}$ models.

By using quartz for the canister end plate and perforated plates so that these transparent elements had the same refractive index as the mineral oil, it was possible to obtain LDV data for the component normal to those surfaces much closer than other investigators could. In this configuration, this component corresponds to the axial direction. 
Contours for rms axial fluctuations normalized by the inlet bulk velocity are displayed in Figure 23. At the inlet jet, values are moderate, consistent with the inlet profile shown earlier in Figure 18. In the rest of the flow, $\mathrm{u}^{\prime} / \mathrm{Vb}_{\mathrm{b}}$ in (denoted $\mathrm{u}^{\prime} / \mathrm{U}$ in the figure legend) is low with a slight increase in the region of the main recirculating eddy. With the exception of the outer region $(\mathrm{r} / \mathrm{D}>\sim 7)$, values immediately upstream of the perforated plate are less than one per cent of $\mathrm{V}_{\mathrm{b}}$,in.

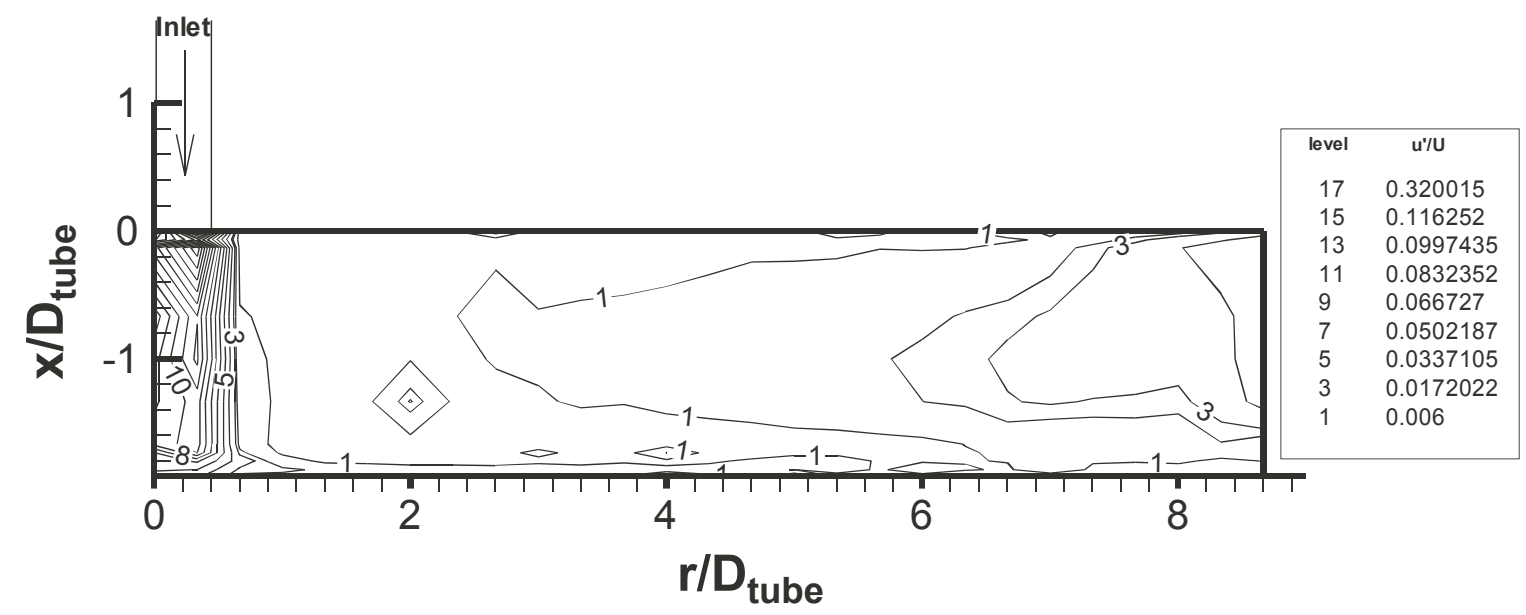

Fig. 23. Distribution of root-mean-squared axial velocity component $u^{\prime} / \mathrm{V}_{b}$,in for a semi-confined impinging jet and its surroundings, $\operatorname{Rejet}=2510$.

The rms radial velocity fluctuations are generally considerably larger than the rms axial fluctuations. Although a local Reynolds number for the bulk outflow between the plates would vary from $\operatorname{ReD}_{, \mathrm{h}}\left(=0.25 \operatorname{Rejet}_{\mathrm{j}}(\mathrm{r} / \mathrm{D})\right) \approx 1260$ at $\mathrm{r} / \mathrm{D}=1 / 2$ to zero at $\mathrm{r}_{\mathrm{o}}$, the turbulent fluctuations remain significant; there is no evidence of a laminarization phenomenon. Figure 24 presents the normalized distribution of $\mathrm{v}^{\prime}$. High values are seen in the wall jet and moderate values mostly in its wake region or the half of the plenum near the wall jet. Moderate to high values also appear near the perforated plate in the region directly affected by the main recirculating eddy. Near the perforated plate in the range $0<\mathrm{r} / \mathrm{D}<7, \mathrm{v}^{\prime}$ is still moderate while $\mathrm{u}^{\prime}$ is low. For the most part, $\mathrm{v}^{\prime}$ is the same order or larger than the mean radial $\mathrm{V}$ so the local turbulence intensities $\mathrm{v}^{\prime} / \mathrm{V}\{\mathrm{r}, \mathrm{x}\}$ are of the order of 100 percent or greater, i.e., "large" turbulence levels. These large relative values of $\mathrm{v}^{\prime}$ imply the likelihood that there are considerable fluctuations in the incident angle as the flow approaches the holes in the perforated plate; this situation differs significantly from flow conditioning applications of perforated plates in wind tunnels. 


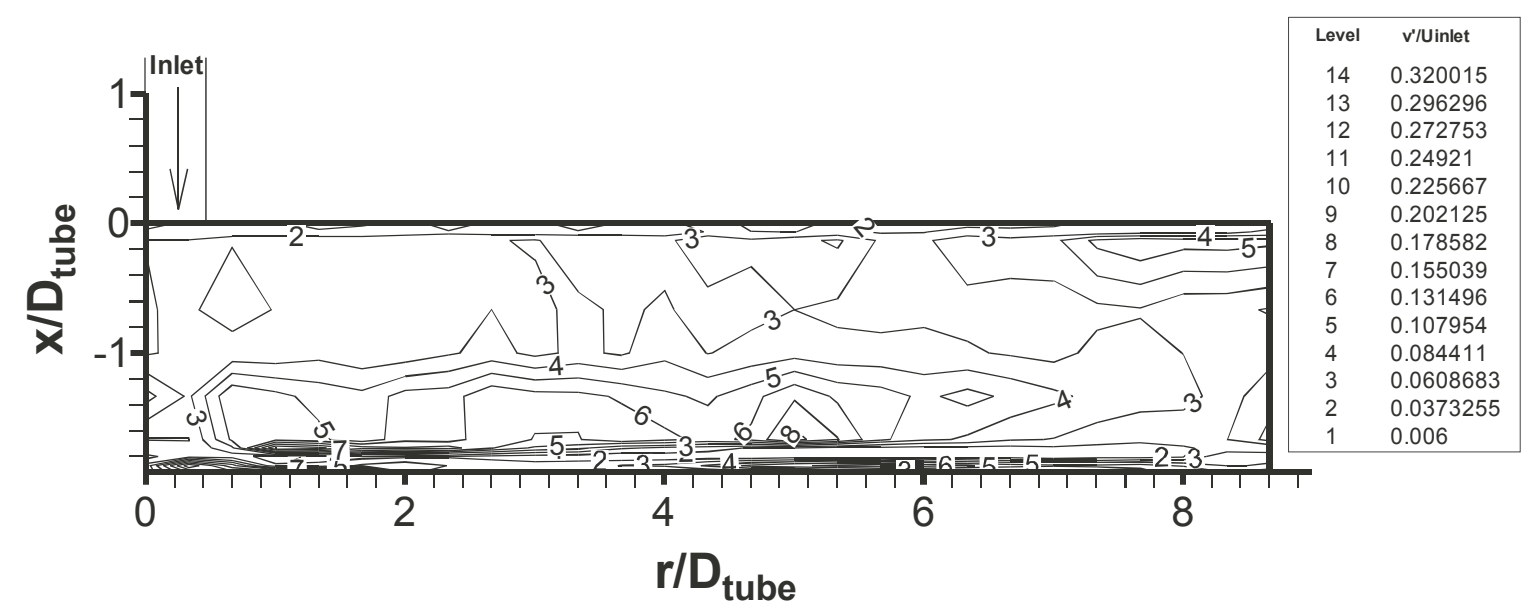

Fig. 24. Distribution of root-mean-squared radial velocity component $\mathrm{v}^{\prime} / \mathrm{V}_{\mathrm{b}}$,in for a semi-confined impinging jet and its surroundings, $R_{j}$ et $=2510$.

Flow visualization As shown by the velocity vectors in Figure 22, the eddy structure observed in the plenum region of the 0.6 -scale MIR model with oil flow exhibited only a single torroidal vortex near the outer radius at $\mathrm{Re} \approx 2500$. On the other hand, the structure observed in the water flow apparatus (with a 4\% open area perforated plate, similar to that in the oil flow apparatus) at the same Reynolds number showed two counter-rotating toroidal vortices (Figures 8 and 9). Therefore the difference in eddy structure was examined by conducting a series of flow visualization experiments at varying flow rates in both the oil flow and water flow models.

Flow visualization in the oil flow apparatus was achieved by injecting small air bubbles in the inlet flow line. The results were recorded photographically. Various camera shutter speeds were used to record bubble path lines. The results shown in Figure 25 were obtained using a duration of one second for $\operatorname{Re} \approx 1650$, and $1 / 2$ second for $\operatorname{Re} \approx 2200$ and $\operatorname{Re} \approx 4200$. The photographic and lighting techniques were the same as for flow visualization in the water flow apparatus as described earlier in this report . 

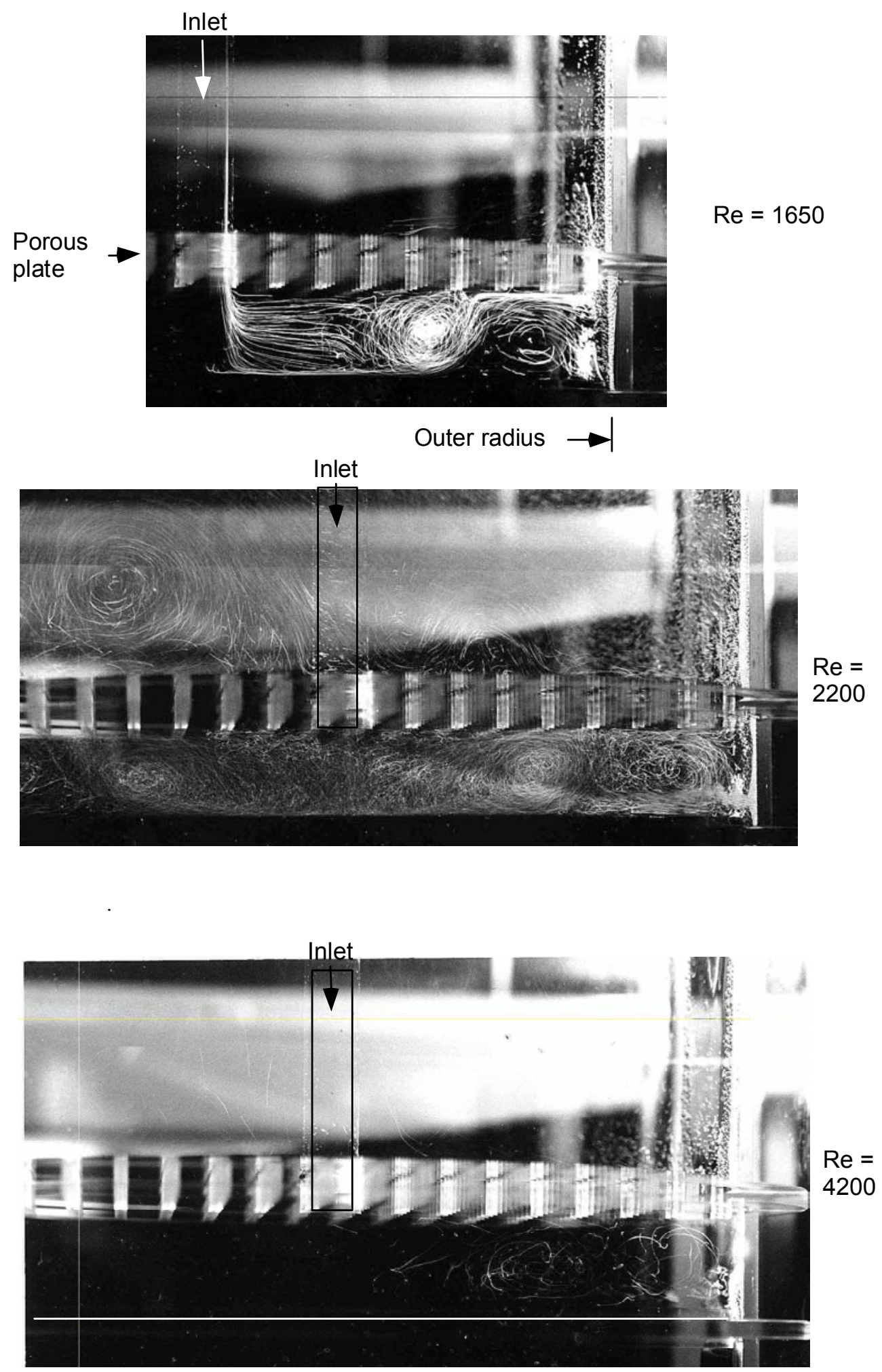

Fig. 25. Eddy structure visualized in plenum region of 0.6 -scale model. 
Flow visualization in the oil flow apparatus clearly shows a double vortex structure for inlet tube Reynolds numbers less than approximately 2,100 . The double vortex structure is shown in the photograph for $\operatorname{Re} \approx 1650$ in Figure 25. The eddy structure changes from a double structure to a single vortex between $\operatorname{Re} \approx 2100$ and $\operatorname{Re} \approx 2300$. This transition is characterized by flow which oscillates non-periodically from a double vortex structure to a single vortex structure. The photograph for $\mathrm{Re} \approx 2200$ shows part of the wall jet flow recirculating in an inner vortex and part of the flow proceeding along the wall to the outer vortex. The photograph for $\operatorname{Re} \approx 4200$ shows a single vortex structure.

The flow visualization experiments conducted in the oil flow apparatus implied that a similar transition in eddy structure should exist in the water flow apparatus, but at a higher Reynolds number. Without simulated fuel elements downstream of the perforated plate, the transition occurred at $\operatorname{Re} \approx 2900$ to 3,200. However, with the simulated fuel elements in place, the double eddy structure was observed for all inlet flow rates up to $\operatorname{Re} \approx 4500$, the maximum achievable in the apparatus. It is concluded that the presence of the simulated fuel elements could have induced the wall jet flow to separate from the wall and form the double eddy structure. However, time (and funding available) did not permit conducting comparable experiments in the oil flow apparatus with simulated fuel elements installed. The effects of these changes in plenum flow pattern on convective mass transfer with the fuel elements remains unclear.

Effect of perforated plate Holes in the perforated bottom plates of typical basket designs have length-to-diameter ratios (L/Dhole) of about 0.5 to 5 . Conceptually, one could attempt to estimate the effects of such plates on the mean flow magnitude and direction plus the turbulence components by applying the method of Taylor and Batchelor [1949] who considered gauze screens. However, it is not clear whether their approach would be valid for perforated plates with $\mathrm{L} / \mathrm{D}$ hole greater than the order of one. In the present experiment, $\mathrm{L} / \mathrm{Dhole} \approx 3.2$ which is an intermediate value for typical designs of canister baskets.

In the plenum region upstream of the perforated plate, the radial velocity component is generally greater than the axial component, except in the recirculating eddy, and the fluctuating radial component is much greater than the axial counterpart. As shown in Figure 22 one set of measurements was obtained downstream of the hole, essentially at the entrance of the basket which would carry an array of fuel rods. In the radial direction these data were taken at the same location as the holes but in the "spanwise" or circumferential direction they were offset slightly. The locus of the hole centers was along $\mathrm{y} \approx 7.5 \mathrm{~mm}$ and the data were taken at $\mathrm{y} \approx 10.95 \mathrm{~mm}$; the axial location was about $6.5 \mathrm{~mm}$ downstream of the plate. Thus, relative to the holes the 
measurements were at about 1.7 diameters from their outlets and at a radius of about 0.9 diameters; we believe these locations would have provided data characteristic of the expanding jets from the holes at their outer edges.

If the flow through the holes were uniform, the average bulk velocity $\mathrm{Vb}$,hole, avg would have been about $0.16 \mathrm{~m} / \mathrm{sec}$, giving a hole Reynolds number of about forty. These limited downstream data give $0.136<\mathrm{U}<0.166 \mathrm{~m} / \mathrm{sec}$ or a ten per cent variation; the lower values were near the center of the simulated canister. As one sees from Figure 22, the flow is predominantly parallel to the axis of the canister; the radial mean velocity was about one per cent of the axial component (or less) except near the hole nearest the center (about twelve per cent there). Relative to $\mathrm{Vb}_{\mathrm{b}}$,hole,avg, the rms axial fluctuations were about 3 to 7 per cent and the radial ones were about 0.5 to 1 per cent. In absolute values, upstream of the plate the radial fluctuations ranged from about 0.09 to $0.18 \mathrm{~m} / \mathrm{sec}$ and in the jets downstream the range was about 0.0008 to $0.002 \mathrm{~m} / \mathrm{sec}$, a reduction of about two orders-of-magnitude in the radial fluctuations. The reduction for the axial fluctuations was approximately a factor of four.

The apparent effects of the perforated plate at these conditions is to convert the predominantly radial mean flow in the plenum region to an axial flow and to reduce turbulence substantially. Since the turbulence of the jets provides the initial freestream turbulence relative to fuel elements it is likely that there would be a substantial effect on the convective mass transfer (drying or passivation) of the elements. However, it would be a mistake to generalize this observation to other conditions. Taylor and Batchelor [1949] show the effects vary with grid resistance which, in turn, varies with both hole configuration and Rehole. Additional measurements are desirable, both with the present model and with other conditions.

\section{CONCLUDING REMARKS}

With the University of Idaho, Ohio State University and Clarksean Associates, this program has the long-term goal to develop reliable predictive techniques for the energy, mass and momentum transfer plus chemical reactions in drying / passivation (surface oxidation) operations in the transfer and storage of Spent Nuclear Fuel (SNF) from wet to dry storage. Such techniques are needed to assist in design of future transfer and storage systems, prediction of the performance of existing and proposed systems and safety (re)evaluation of systems as necessary at later dates. 
Many fuel element geometries and configurations are accommodated in the storage of spent nuclear fuel. Consequently, there is no one generic fuel element / assembly, storage basket or canister and, therefore, no single generic fuel storage configuration. One can, however, identify generic flow phenomena or processes which may be present during drying or passivation in SNF canisters. The objective of the INEEL tasks was to obtain fundamental measurements of these flow processes in appropriate parameter ranges.

With the University of Idaho, an idealization of a combined drying and passivation approach has been defined in order to investigate the generic flow processes. This simulation includes flow phenomena that occur in canisters for high-enrichment and medium-enrichment fuels, where fuel element spacing in the canister is increased as compared with low enrichment fuel. Canister diameter was taken as $46 \mathrm{~cm}$ (18 in.) and a single basket of about 1.3 meters ( $4 \mathrm{ft}$. .) length was considered. A long central tube ("dip tube") served as the inlet as in one earlier concept for a passivation process; while this concept apparently has not yet been selected for application, it provides an excellent example of the coupled, complex phenomena which may be present in canister flows. Suggested design flow rates for this hypothesized application indicate that the Reynolds number in the inlet tube would be expected to be between 2500 and 5000, i.e., relatively low.

The local distributions of convective mass transfer characteristics (drying/passivation) are expected to depend on the freestream turbulence in the flow around stored fuel elements. The magnitudes of this turbulence depend on the turbulence distributions on the upstream side of the perforated basket support plate ("inlet plenum") and, in turn, in the impinging jet and in the inlet tube.

To date the INEEL efforts have emphasized two tasks:

\section{- Overall flow visualization for a variety of configurations and}

- Pointwise turbulence and velocity measurements with two-component laser Doppler velocimetry in the unique INEEL Matched-Index-of-Refraction flow system

This information can assist engineers in understanding variations of surface drying and passivation through an array and approaches to modify designs to counter non-uniformities and to improve distribution, as well as providing bases for assessment of computational fluid dynamics codes proposed for the purpose. 
A water-flow experiment with a 3/4-scale model (relative to the idealized canister) has been used for overall flow visualization and velocity measurements, with and without an array of simulated fuel elements. Its purposes were to obtain initial indications of the gross flow behavior and to investigate the circumferential periodicity of the flow. Standard flow visualization techniques, using fluorescent dyes and neutrally buoyant particles illuminated by laser and white light, were employed. Observations have been made with perforated plates (representing basket support plates) having three hole geometries, with and without simulated fuel elements. Streamline and velocity vector plots are presented. The flow patterns exhibited are very complex and provide a non-uniform flow distribution along the simulated fuel elements.

Experiments were conducted to determine the canister flow field with three perforated plates with open areas of 50\%, 8\% and 4\% ("porosities"). Experiments reported are for Reinlet $=2,500$ \pm 100 . Although laminar flow in a smooth tube can occur at Reynolds number as high as approximately 40,000, it is believed that the tube is sufficiently long and the entrance conditions sufficiently abrupt to insure that turbulent flow exits the tube and impinges on the plate. The flow is approximately circumferentially periodic and symmetrical about cross-sections through the four outer tubes for experiments using each of the three perforated plates. This observation implies that numerical predictions and detailed measurements can concentrate on an 1/8-th sector of the idealized geometry rather than the full cross section.

With a $50 \%$ open area plate, the flow pattern is comparable to that of a submerged impinging jet on a semi-infinite plate with formation of a large vortex and entrainment and recirculation through the holes in the perforated plate near the center. Downstream flow is primarily through the outer holes. A single main recirculating eddy forms, encompassing the plenum and part of the region for the element array.

With the $4 \%$ and $8 \%$ open area plates, recirculation regions occur downstream of the perforated plate and two main recirculation zones (plus a small corner flow vortex) form upstream of the perforated plate. Flow does not reenter the inlet plenum through the smaller open area plates as it does with the $50 \%$ open area plate. In these cases the flow pattern in the inlet plenum has some features of a confined impinging jet [Morris and Garimella, 1996].

The Reynolds number in the array downstream of the plate based on hydraulic diameter is approximately 70 for Reinlet $=2,500$. With the tubes present, vortices persist for the majority of the tube bundle length and the flow only starts to approach a fully-developed laminar profile near the exit of the bundle ( $\mathrm{L} / \mathrm{D}_{\mathrm{h}} \approx 6$, where $\mathrm{D}_{\mathrm{h}}$ is the hydraulic diameter). The flow distribution is 
uneven near the surface of the simulated fuel elements. Higher velocity is observed near the surface towards the canister centerline than towards the outside where, except near the perforated plate, the velocity is an order-of-magnitude lower. These experiments show flow across the simulated elements and resultant vortex shedding, although the vortices do not show the clear alternating pattern of a Karman vortex street [Tritton, 1977].

Velocity and turbulence distributions were measured with laser Doppler velocimetry in the unique INEEL Matched-Index-of-Refraction (MIR) flow system. An advantage of this facility is the capability of obtaining optical fluid mechanics data without optical distortion and without disturbing the flow by inserting a physical sensor. A 0.6-scale model of the idealized canister was employed with dimensions as follows: plenum spacing $H / D=s / D=2$, canister internal radius $\mathrm{r}_{\mathrm{o}} / \mathrm{D}=9$, pitch of holes in the perforated plate $\mathrm{ph} / \mathrm{D}=1$ and hole diameter $\mathrm{dh} / \mathrm{D}=1 / 4$ in a square pattern. Most measurements were taken at $R e_{i n l e t}=2510$. This part of the study concentrated on flow in the inlet tube, the impinging jet and flow upstream of the perforated plate (inlet plenum). Flow at the exit of the inlet tube was determined to be one that was intermittently turbulent and laminar, i.e., undergoing axial transition. However, the mean velocity profile still had predominantly laminar character.

Measurements included the mean distributions of radial and axial velocity components, $V$ and $U$, and their root-mean-squared fluctuations, $v^{\prime}$ and $u^{\prime}$. These data would be suitable for comparison to predictions from Reynolds stress models [Hanjalic, 1999] or k- $\varepsilon-\mathrm{v}^{2}$ models.

Main features of the flow in the inlet plenum upstream of the perforated plate are the high velocity impinging jet, the thin expanding wall jet, a large recirculating eddy near the outer wall of the canister and relatively low velocities near the perforated plate. The flow in the wall jet remains attached until it impinges on the outer wall of the plenum region and induces the main recirculating eddy. Only a single major eddy is seen rather than the two observed with the water flow visualization. Outside the impinging and wall jets, flow is relatively quiescent with velocities about an order-of-magnitude less than the inlet velocity. In the plenum region upstream of the perforated plate, the radial velocity component at a point is generally greater than the axial component, except in the recirculating eddy.

The rms radial velocity fluctuations are generally considerably larger than the rms axial fluctuations. High values of $\mathrm{v}^{\prime}$ are seen in the wall jet and moderate values mostly in its wake region or the half of the plenum near the wall jet. Moderate to high values also appear near the perforated plate in the region directly affected by the main recirculating eddy. Near the perforated 
plate in the range $0<\mathrm{r} / \mathrm{D}<7, \mathrm{v}^{\prime}$ is still moderate while $\mathrm{u}^{\prime}$ is low. For the most part, $\mathrm{v}^{\prime}$ is the same order or larger than the mean radial $\mathrm{V}$ so the local turbulence intensities $\mathrm{v}^{\prime} / \mathrm{V}\{\mathrm{r}, \mathrm{x}\}$ are of the order of 100 percent or greater, i.e., "large" turbulence levels. The large relative values of $v^{\prime}$ imply the likelihood that there are considerable fluctuations in the incident angle as the flow approaches the holes in the perforated plate; this situation differs significantly from flow conditioning applications of perforated plates in wind tunnels.

Comparison of the present results shows that flow in the plenum region upstream of the perforated basket support plate is sensitive to the inlet flow characteristics in the expected range of Reynolds number. What has not been determined is whether turbulent, laminar or transitional inlet flow is preferable for desired drying characteristics (or whether it matters); further measurements are required to answer this question.

It is recommended that the present data be used to assess capabilities of existing and proposed CFD (computational fluid dynamics) codes which are intended to predict behavior in SNF canisters. While proprietary codes for predicting drying supposedly have been validated for the nuclear reactor industry, it is not clear that these "validations" have been at appropriate Reynolds numbers and geometrical ranges or how close the predictions were to any measurements (since they are "proprietary"). It would be desirable to test such codes by comparison to the present measurements.

It is apparent from these experiments that, despite the conceptual simplicity of the canister design, the flow pattern is very complex. Of possible concern is the uneven flow distribution near the surface of the simulated fuel elements as demonstrated in the water flow visualization experiments. Higher velocity is observed near the element surface towards the canister centerline than towards the outside where, except near the perforated plate, the velocity is an order-ofmagnitude lower. A more even flow distribution is expected to provide more uniform drying and passivation around the elements. Possible design changes that could provide more uniform distributions (if that is desirable) include (1) an optimized distribution of perforated plate holes , (2) a longer inlet plenum length (to decrease radial velocity tangent to the perforated plate which will presumably provide a more even flow distribution) and (3) a higher packing density of fuel elements, where criticality considerations allow. The canister and plate geometry could be optimized using a CFD code once it has been verified that the code can reasonably predict the present and other needed experiments.

Additional lessons learned for design of drying/passivation systems may be summarized 
- $\quad$ Bypass flow routes should be avoided

- Flow distribution should be controlled

- Assumptions of uniform flow could be misleading

- Previous measurements of flow through perforated plates for wind tunnel conditioning are not likely to be applicable to basket support plates

- Modifications of basket support plates and lower regions of canisters could be useful to distribute turbulent flow to the elements

- $\quad$ Do not trust commercial, general purpose CFD codes using popular turbulence models --Test!

\section{RECOMMENDATIONS FOR FURTHER WORK}

The goals of the INEEL experimental portion of this study have been to be to answer the scientific needs identified above and to guide code development and to assess code capabilities for treating the generic flow processes in the idealized configuration(s). The main objective of the INEEL tasks is to obtain fundamental measurements of the basic velocity and turbulence fields in generic idealizations of flow processes in SNF canisters during transfer and/or storage.

The present measurements have revealed complex flow patterns which are expected to be difficult to predict properly with commercial CFD codes. In some regimes predictions of flow patterns, velocities and turbulence levels would be uncertain. Uncertainties in flow lead to uncertainties in convective mass transfer locally along fuel elements, leading further to uncertainties in drying (and passivation) rates locally.

In order to assess the performance of proposed codes, it would be appropriate to compare their predictions to the present results. However, additional fundamental measurements would be appropriate to cover the fundamental flow processes of Figure 2 not yet treated in detail. Consequently, the emphases of further work should concentrate on experiments examining 
- the effects of a sparsely perforated plate on the turbulence field, downstream and upstream

- decay of turbulence fields in simulated fuel element arrays, both sparse (highly-enriched and moderately-enriched) and dense (low enrichment)

plus related velocity fields. These studies would address the topics from the initial proposal that were deferred due to a 45 percent reduction in funds awarded relative to the budget originally proposed.

The existing MIR model for these fluid physics experiments can be used, with and without simulated fuel elements. Also the flow visualization model can also be employed for rapid examination of a variety of potential configurations.

Since suggested design flow rates for SNF storage/passivation applications indicate that the inlet Reynolds number would be expected to be between 2500 and 5000, the experiments should be conducted with inlet flow rates giving this range. The resulting Reynolds number in the fuel element array would be of the order of 100, giving strong potential for laminarization as the flow passes through the array. As shown in the present overall flow visualizations, continuous cross flow occurs in the array as the exit is approached.

Selection of models with simulated fuel elements (high-, moderate- or low -enrichment) would be conducted in coordination with technical representatives of the Spent Nuclear Fuel programs.

Measurements should be obtained by Particle Tracking Velocimetry (PTV) with the Moving Particle Tracking system (MPT) and by laser Doppler velocimetry (LDV). The MPT system developed by Profs. Brodkey and Guezennec at Ohio State - in another subtask of the present grant would give additional possibilities and capabilities for quantitative 3-D flow visualization and measurements of particle fluxes and individual particle paths. The particle tracks would represent the motion of particular species in the flow. The MPT system could be employed to obtain quantitative flow field visualization in both the "plenum" and element array regions. In addition to the velocity field and particle flux fields, data could be reduced to examine individual particle tracks as suggested in the original proposal. This information can assist engineers in understanding variations of surface passivation through an array and approaches to modify designs to counter the non-uniformities and to improve distribution.

In order to simulate portions of the generic transport problem for an oxidizing component of a mixture, individual particles might be tracked from introduction via the central tube flow to multiple 
interactions with the array surfaces. New PTV techniques are being developed by Profs. Guezennec and Brodkey of Ohio State with the INEEL staff as part of the scope of the parallel work in order to allow this individual particle tracking (IPT). Current PTV methods concentrate on small fields of view (e.g., $8 \times 8 \times 8 \mathrm{~cm}^{3}$ [Kasagi and Matsunaga, 1995]). For IPT the field of view must track erratic particles in all three coordinate directions from the deduced instantaneous particle motion; in the proposed experiment, tracking distances of the order of a meter would be involved. The individual particle tracks and additional statistics are determined by analyzing off-line the motion of all particles which remain in the field of view as individual particles are tracked. The overall statistics for the particle transport would be derived from analysis of a large number of IPT records at the same inlet flow conditions.

Also the overall flow field would be examined quantitatively via flow visualization deduced from application of the MPT system. Equipment for the new MPT technique includes a PCcontrolled camera system mounted on a longitudinal traversing system controlled by the same computer. Traversing can be programmed at variable speeds to about $15 \mathrm{~cm} / \mathrm{sec}$. The MPT system will be based on two 512 x 512 CCD cameras with framing rates of 60 fps with a C-mount lens. They will be mounted on a four foot linear traversing system with one tilting stage per camera. Illumination will be by a collimated beam strobe synchronized to the cameras. System control will be via P450-128MB computer systems and imaging will be by $\mathrm{P} 450-128 \mathrm{MB}$ computer systems. Control and imaging / data reduction programs are extensions of existing programs by Profs. Guezennec and Brodkey, written in the $C$ language with Windows user interface. When held in a fixed position the MPT method reverts to the successful PTV technique of Profs. Guezennec and Brodkey [Guezennec and Kiritis, 1990; Choi, Guezennec and Brodkey, 1992; Kent et al., 1993; Guezennec et al., 1994 ]. The flow visualization and related overall statistics will be derived from analysis of a large number of MPT records at the same inlet flow conditions. Typical results would include mean streamlines and velocity vectors plus qualitative turbulence quantities corresponding to and extending those measured with the LDV system. Attempts would also be made to obtain transient flow patterns, as in the wakes of the simulated fuel elements.

To supplement the velocity field data and particle tracks deduced via the MPT/PTV methods, measurement of instantaneous velocity components would continue by LDV at fixed positions, primarily in the array. Mean velocities and mean turbulence statistics would be determined from these time series. Thus, these results would give an Eulerian view while the new IPT method is a complementary laGrangian approach. The LDV is a two-component fiber-optics-based version mounted on a three-directional traversing mechanism. Since the traverse follows rectangular coordinates and the model geometry is circumferentially periodic, the LDV can be positioned to 
measure radial and longitudinal velocity components at one point and, by repositioning, circumferential and longitudinal components at an equivalent point in another region of the array. Typical results would include time-resolved, pointwise distributions of the mean velocities, $\mathrm{U}, \mathrm{V}, \mathrm{W}$, and their Reynolds stress components. The time series would also be available for spectral and wavelet analysis [leWalle, Ashpis and Sohn, 1997; Resnikoff and Wells, 1998]. The upstream effect of the semi-confinement (by the basket support plate) on flow and turbulence fields in the "plenum" region would be examined via experiments varying the distance H/D between the perforated plate and the solid plate representing the bottom of a canister.

Additional parameters or phenomena which should be investigated systematically include

- inlet Reynolds number

- inlet geometry

- fuel rod loading

- hole pattern and length in the basket support plate

- positioning of the basket support plate $(\mathrm{H} / \mathrm{D})$

The results of such studies should provide new fundamental knowledge on

- $\quad$ statistics of particle and passive scalar transport from laGrangian measurements in a rod bundle

- laminarization of turbulent flows entering an array of elements, in combined cross and longitudinal flow

- turbulence behavior in the surroundings of a semi-confined impinging jet 


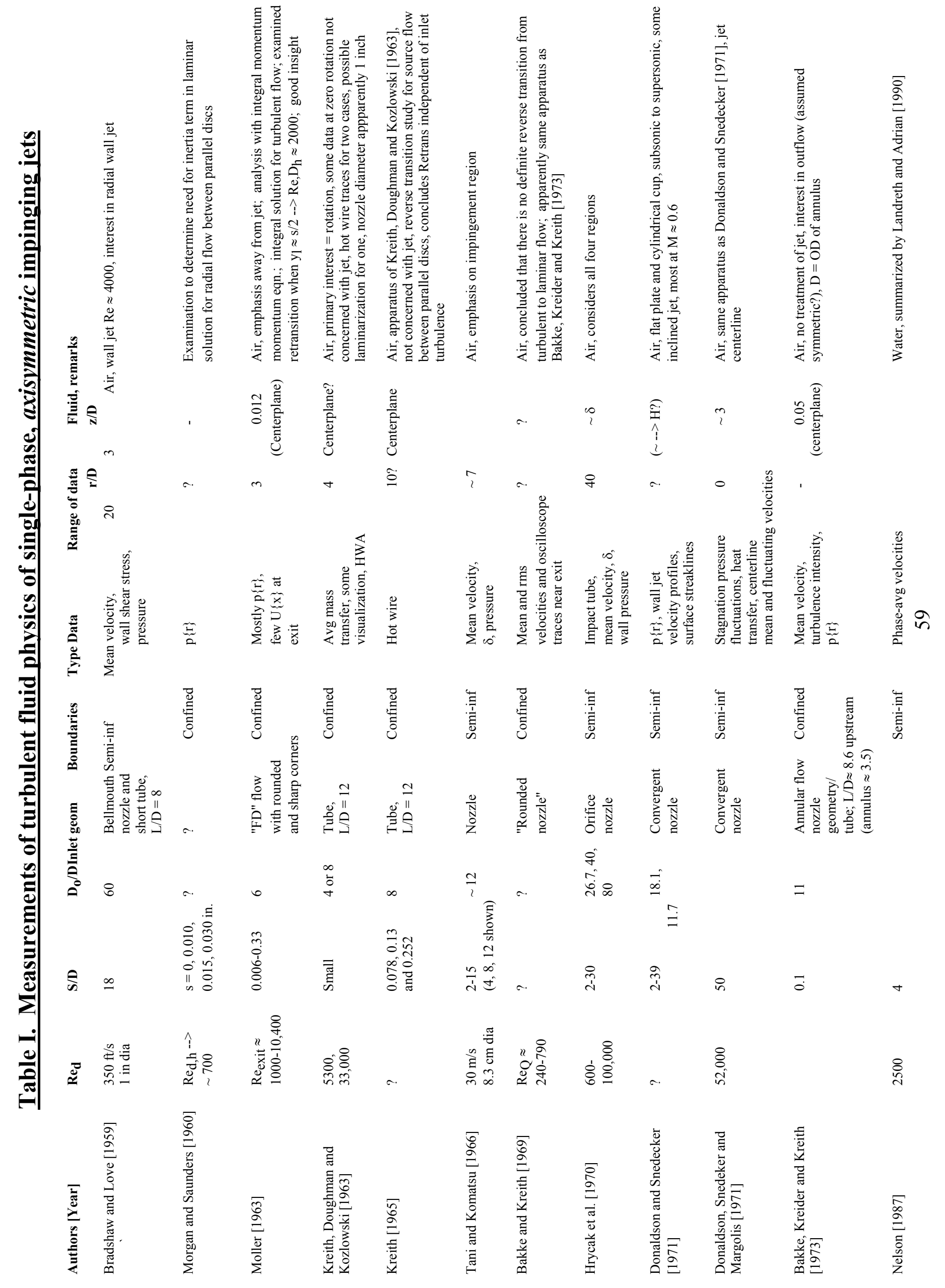




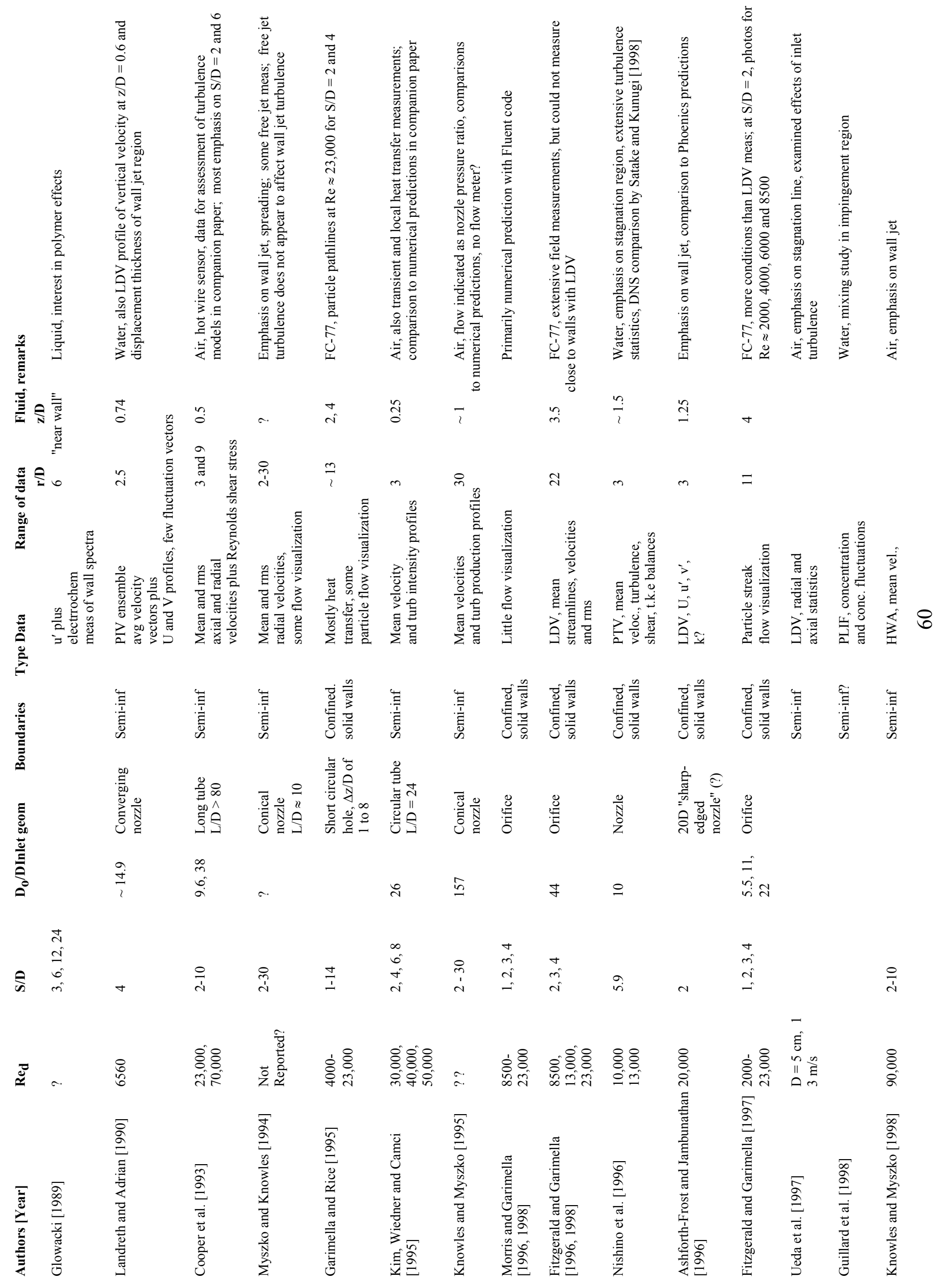




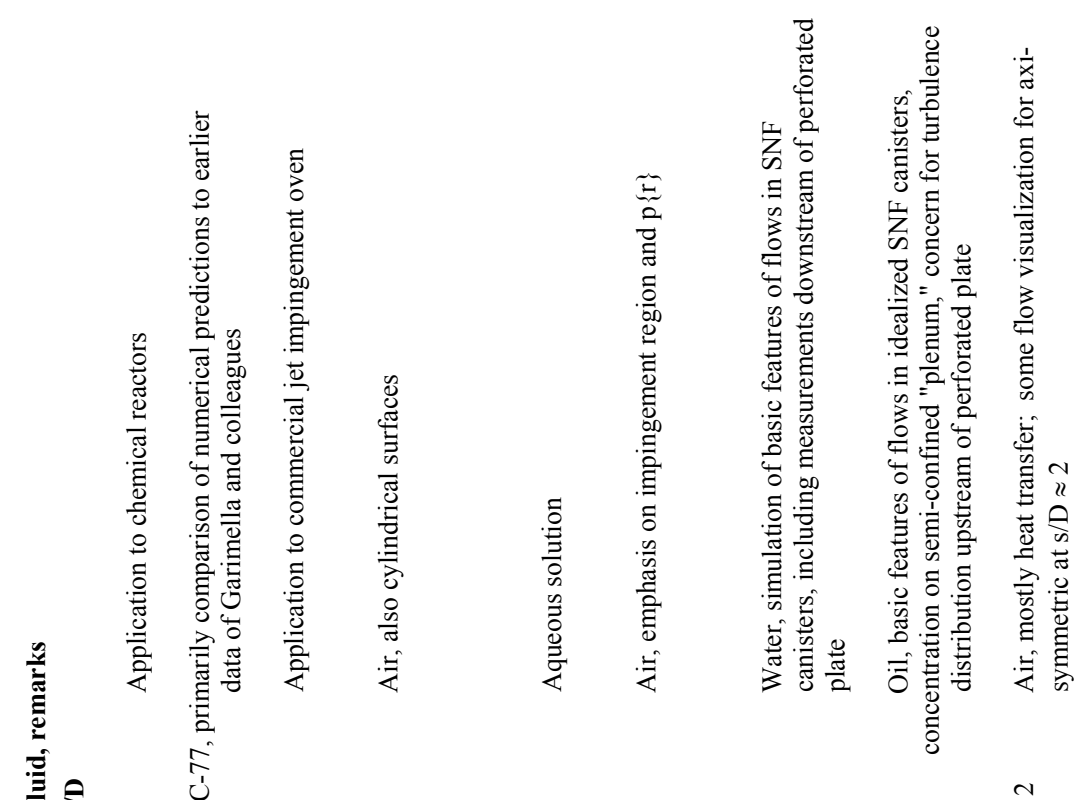

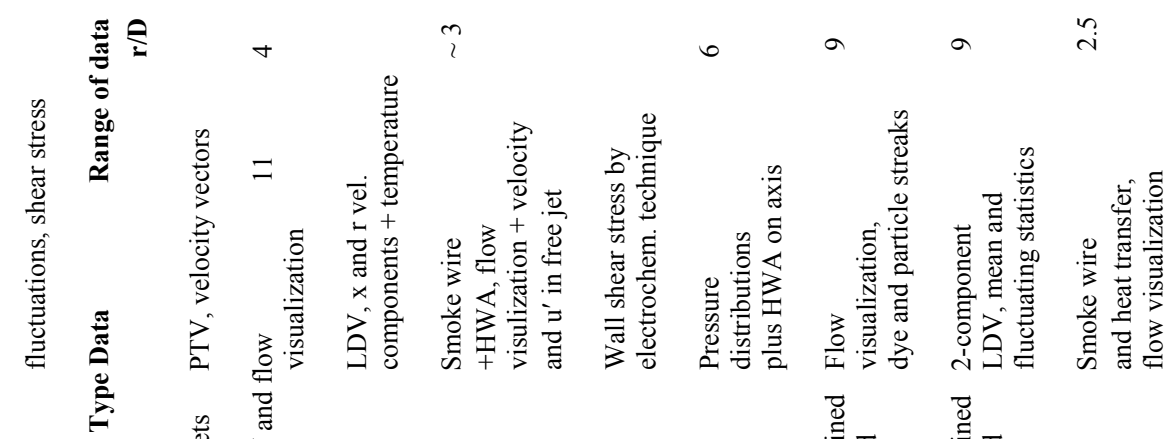

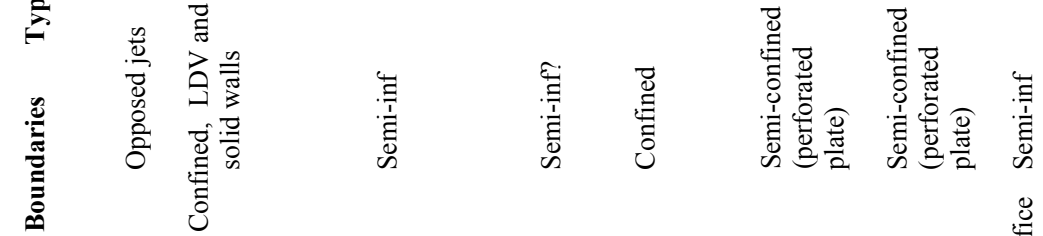

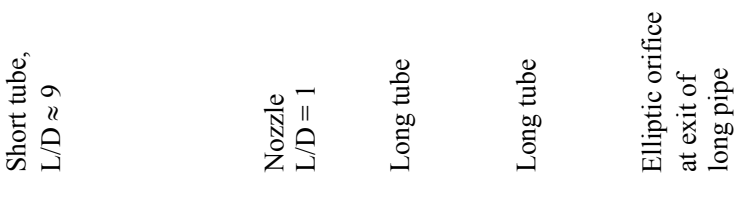

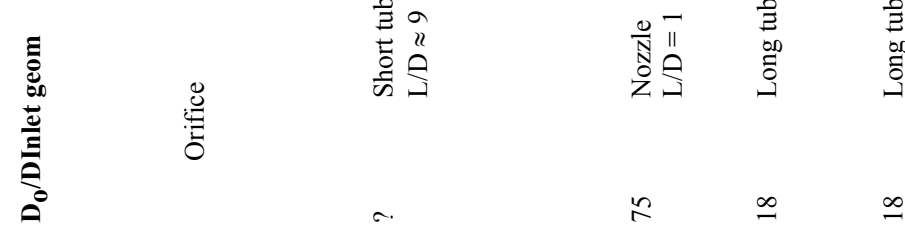

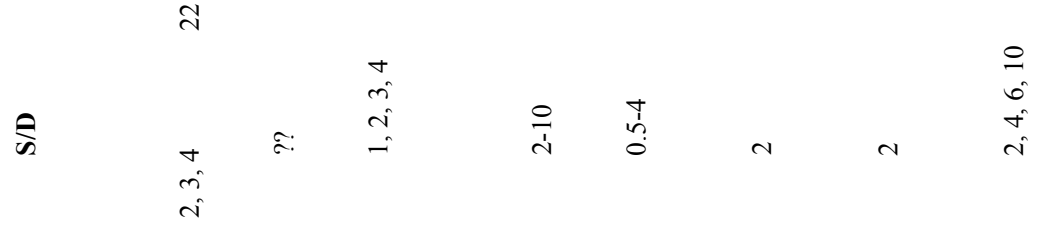

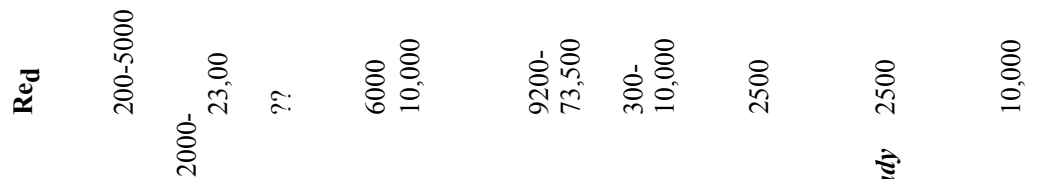

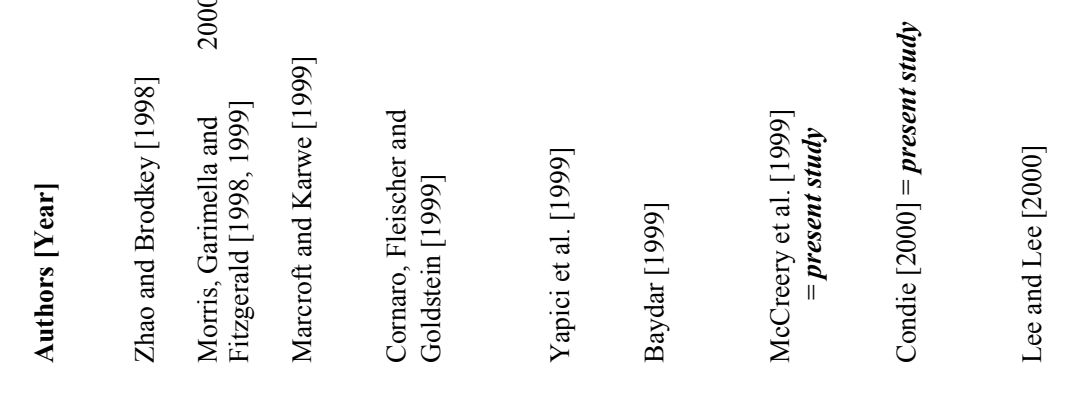




\section{REFERENCES CITED}

Achenbach, E., 1989. Heat transfer from a staggered tube bundle in cross-flow a high Reynolds numbers. Int. J. Heat Mass Transfer, 32, pp. 271-280.

Adrian, R. J., 1986. Multi-point optical measurements of simultaneous vectors in unsteady flow - a review. Int. J. Heat Fluid Flow, 7 , pp. 127-145.

Adrian, R. J., 1991. Particle-imaging techniques for experimental fluid mechanics. Ann. Rev. Fluid Mech., 23, pp. 261304.

Adrian, R. J. (Ed.), 1993. Selected papers on laser Doppler anemomentry. New York: SPIE.

Albrecht, H.-E., 1986. Laser-Doppler-Strömungsmessung. Berlin: Akademie.

Amano, R. S., and H. Brandt, 1984. Numerical study of turbulent axisymmetric jets impinging on a flat plate and flowing into an axisymmetric cavity. J. Fluids Engr., 106, pp. 410-417.

Ashforth-Frost, S., and K. Jambunathan, 1996. Numerical prediction of semi-confined jet impingement and comparison with experimental data. Int. J. Num. Methods in Fluids, 23, pp. 295-306.

Bakke, E., and F. Kreith, 1969. Inverse transition in radial diffusers. ASME paper 69-HT-33.

Bakke, E., J. F. Kreider and F. Kreith, 1973. Turbulent source flow between parallel stationary and co-rotating disks. $J$. Fluid Mech., $\underline{58}$, pp. 209-231.

Barsamian, H. R., and Y. A. Hassan, 1996. Large eddy simulation of tube bundle geometries using the dynamic subgrid scale model. Thermal Science of Advanced Steam Generators/Heat Exchangers, ASME NE-Vol. 19, pp. 49-56.

Baughn, J. W., and S. Shimizu, 1989. Heat transfer measurements from a surface with uniform heat flux and an impinging jet. J. Heat Transfer, 111, pp. 1096-1098.

Baydar, E., 1999. Confined impinging air jet at low Reynolds number. Exp. Thermal Fluid Sci., 19, pp. 27-33.

Beale, S. B., and D. B. Spalding, 1998. Numerical study of fluid flow and heat transfer in tube banks with streamwiseperiodic boundary conditions. Trans., Can. Soc. Mech. Eng., 22, pp. 397-416.

Becker, S., 2001. Advanced laser velocimetry technique. LSTM Erlangen, seminar presented at INEEL, August.

Becker, S., C. M. Stoots, K. G. Condie and D. M. McEligot, 1999. Reynolds stress development in the viscous layer of a transitional boundary layer. Proc., IUTAM Symposium on Laminar-Turbulent Transition, Sedona, Ariz., September.

Becker, S., C. M. Stoots, K. G. Condie, F. Durst and D. M. McEligot, 2002. LDA-measurements of transitional flows induced by a square rib. J. Fluids Engineering, in press.

Behnia, M., S. Parneix, Y. Shabany and P. A. Durbin, 1999. Numerical study of turbulent heat transfer in confined and unconfined impinging jets. Int. J. Heat Fluid Flow, 20, pp. 1-9.

Bendat, J. S., and A. G. Piersol, 1986. Random data analysis and measurement procedures. New York: John Wiley \& Sons.

Berner, C., F. Durst and D. M. McEligot, 1984. Flow around baffles. J. Heat Transfer, 106, pp. 743-749.

Bradshaw. P., 1965. The effect of wind-tunnel screens on nominally two-dimensional boundary layers. J. Fluid Mech., $\underline{22}$, pp. 679-687. 
Bradshaw, P., and E. M. Love, 1959. The normal impingement of a circular air jet on a flat surface. Tech. report A.R.C. R. \& M. 3205, Aeronautical Research Council (England).

Budwig, R., 1994. Refractive index matching methods for liquid flow investigations. Exp. Fluids, 17, pp. 350-355.

Budwig, R., D. Elger, H. Hooper and J. Slippy, 1993. Steady flow in abdominal aortic aneurysm models. J. Biomech. Engr., 115, pp. 418-423.

Burd, S. W., and T. W. Simon, 1997. The influence of coolant supply geometry of film coolant exit flow and surface adiabatic effectiveness. ASME paper 97-GT-25.

Burd, S. W., and T. W. Simon, 1998. Measurements of discharge coefficients in film cooling. Proc., Int. Gas Turbine Conf., Stockholm.

Calabrese, R. V., and S. Middleman, 1979. The dispersion of discrete particles in a turbulent flow field. AIChE J., 25, No. 6, pp. 1025-1035.

Chambers, F. W., H. D. Murphy and D. M. McEligot, 1983. Laterally converging flow. Part 2. Temporal wall shear stress. J. Fluid Mech., 127, pp 403-428.

Chen, S. S., and J. A. Jendrzejczyk, 1987. Fluid excitation forces acting on a tube array. Tech. rpt. ANL-85-55.

Choi, W.-C., Y. G. Guezennec and R. S. Brodkey, 1992. In situ calibration and stereo matching for 3-D particle image velocimetry. Bull., Am. Physical Soc., $\underline{36}$.

Condie, K. G., Stoots, C. M., Becker, S., Alahyari, A. A., Durst, F., and McEligot, D. M., 1998. The structure of boundary layer transition induced by a square rib (A new large-scale MIR flow system for measurements of boundary layer transition). Tech. Report INEEL/EXT-98-01039, Idaho National Engineering and Environmental Laboratory.

Cook, N. H., and E. Rabinowicz, 1963. Physical Measurement and Analysis. Palo Alto: Addison-Wesley.

Cooper, D., D. C. Jackson, B. E. Launder and G. X. Liao, 1993. Impinging jet studies for turbulence model assessment I. Flow-field experiments. Int. J. Heat Mass Transfer, $\underline{36}$, pp. 2675-2684.

Corino, E. R., and R. S. Brodkey, 1969. A visual observation of the wall region in turbulent flow. J. Fluid Mech., $\underline{37}$, pp. 1-30.

Cornaro, C., A. S. Fleischer and R. J. Goldstein, 1999. Flow visualization of a round jet impinging on cylindrical surfaces. Exp. Th. Fluid. Sci., 20, pp. 66-78.

Craft, T. J., 1998. Predictions of heat transfer in turbulent stagnation flow with a new second-moment closure. Proc., $2^{\text {nd }}$ Eng. Foundation Conf. Turbulent Heat Transfer, Manchester.

Craft, T. J., L. J. W. Graham and B. E. Launder, 1993. Impinging jet studies for turbulence model assessment - II. An examination of the performance of four turbulence models. Int. J. Heat Mass Transfer, 36, pp. 2685-2697.

Cui, M. M., and R. J. Adrian, 1997. Refractive index matching and marking methods for highly concentrated solidliquid flows. Exp. Fluids, $\underline{22}$, pp. 261-264.

Dianat, M., M. Fairweather and W. P. Jones, 1996. Prediction of axisymmetric and two-dimensional impinging turbulent jets. Int. J. Heat Fluid Flow, 17, pp. 530-538.

Donaldson, C. S., and R. S. Snedeker, 1971. A study of free jet impingement. Part 1. Mean properties of free and impinging jets. J. Fluid Mech., $\underline{45}$, pp. 281-319. 
Donaldson, C. S., R. S. Snedeker and D. P. Margolis, 1971. A study of free jet impingement. Part 2. Free jet turbulent structure and impingment heat transfer. J. Fluid Mech., $\underline{45}$, pp. 477-512.

Downs, H. T., and E. H. James, 1987. Jet impingement heat transfer - A literature survey. ASME paper 87-HT-35.

Durst, F., J. Jovanovic and J. Sender, 1993. Detailed measurements of the near wall region of turbulent pipe flows. Proc., 9th Symp. Turb. Shear Flows, Kyoto, paper 2-2.

Durst, F., Keck, T., and Kleine, R., 1979. Turbulence quantities and Reynolds stress in pipe flow of polymer solutions measured by two-channel laser-Doppler anemometry. Proc., $6^{\text {th }}$ Symp. On Turbulence, Rolla, Mo.

Durst, F., H. Kikura, I. Lekakis, J. Jovanovic and Q. Ye, 1996. Wall shear stress determination from near-wall mean velocity data in turbulent pipe and channel flows. Exp. Fluids, 20, pp. 417-428.

Durst, F., A. Melling and J. H. Whitelaw, 1976. Principles and practise of laser-Doppler anemometry. London: Academic Press.

Ekman, V. W., 1910. On the change from steady to turbulent flow of liquids. Ark.f. Mat. Astron. och Fys., $\underline{6}$, No. 12.

Ezato, K., A. M. Shehata, T. Kunugi and D. M. McEligot, 1999. Numerical prediction of transitional features of turbulent gas flows in circular tubes with strong heating. J. Heat Transfer, 121, pp. 546-555.

Farell, C., and S. Youssef, 1996. Experiments on turbulence management using screens and honeycombs. J. Fluids Engr., 118, pp. 26-32.

Fitzgerald, J. A., and S. V. Garimella, 1996. Flow field measurements in confined and submerged jet impingement. Proc., Nat. Heat Transfer Conf., ASME HTD-Vol. 333, volume 2, pp. 121-129.

Fitzgerald, J. A., and S. V. Garimella, 1997. Flow field effects on heat transfer in confined jet impingement. J. Heat Transfer, 119, pp. 630-632.

Fitzgerald, J. A., and S. V. Garimella, 1997. Visualization of the flow field in a confined and submerged impinging jet. Proc., Nat. Heat Transfer Conf., ASME HTD-Vol. 346, volume 8, pp. 93-99

Fitzgerald, J. A., and S. V. Garimella, 1998. A study of the flow field of a confined and submerged impinging jet. Int. J. Heat Mass Transfer, 41, pp. 1025-1034.

Gardon, R., and J. C. Akfirat, 1965. The role of turbulence in determining the heat transfer characteristics of impinging jets. Int. J. Heat Mass Transfer, $\underline{8}$, pp. 1261-1272.

Garimella, S. V., and R. A. Rice, 1995. Confined and submerged liquid jet impingement heat transfer. J. Heat Transfer, $\underline{117}$, pp. 871-877.

Glowacki, P., 1989. Turbulence characteristics of impinging jets near the deflecting surface. The effect of polymer additives. J. Non-Newtonian Fluid Mech., 33, pp. 25-38.

Goldstein, R. J., and A. L. Behbahani, 1982. Impingement of a circular jet with and without cross flow. Int. J. Heat Mass Transfer, 25, pp. 1377-1382.

Grimison, E. D., 1937. Correlation and utilization of new data on flow resistance and heat transfer for cross flow of gases over tube banks. Trans. ASME, $\underline{59}$, pp. 583-594.

Gritsch, M., A. Schulz and S. Wittig, 1997. Discharge coefficient measurements of film-cooling holes with expanded exits. ASME paper 97-GT-165. 
Gritsch, M., A. Schulz and S. Wittig, 1999. Effect of internal coolant crossflow on the effectiveness of shaped filmcooling holes. Nat. Heat Transfer Conf., Albuquerque.

Guezennec, Y. G., R. S. Brodkey, N. Trigui and J. C. Kent, 1994. Algortihms for fully automated three-dimensional particle tracking velocimetry. Exp. Fluids, 17, pp. 209-219.

Guezennec, Y., and N. Kiritsis, 1990. Statistical investigation of errors in particle image velocimetry. Exp. Fluids, $\underline{10}$, pp. 138-146.

Guillard, F., R. Fritzon, J. Revstedt, C. Tregardh, M. Alden and L. Fuchs, 1998. Mixing in a confined turbulent impinging jet using planar laser-induced fluorescence. Exp. Fluids, 25, pp. 143-150.

Habib, M. A., F. Durst and D. M. McEligot, 1984. Streamwise-periodic flow around baffles. Proc., 2nd Int. Symp. Appl. Laser Anemometry Fl. Mech., Lisbon, July.

Hale, C. A., M. W. Plesniak and S. Ramadhyani, 1999. Film cooling effectiveness for short film cooling holes fed by a narrow plenum. ASME paper 99-GT-036, Int. Gas Turbine Conf., Indianapolis.

Hanjalic, K., 1999. Second-moment turbulence closures for CFD: Needs and prospects. Int. J. Comp. Fluid Dyn., $\underline{12}$, pp. 67-97.

Hay, N., D. Lampard and S. Benmansour, 1983. Effect of crossflows on the discharge coefficient of film cooling holes. J. Engr. Power, 105, pp. 243-248.

Hay, N., S. E. Henshall and Manning, 1994. Discharge coefficients of holes angled to the flow direction. $J$. Turbomachinery, 116, pp. 92-96.

Heyerichs, K., and A. Pollard, 1996. Heat transfer in separated and impinging turbulent flows. Int. J. Heat Mass Transfer, 39 , pp. 2385-2400.

Hrycak, P., 1981. Heat transfer from impinging jets - A literature review. Tech. rpt. AFWAL-TR-81-3504.

Hrycak, P., 1983. Heat transfer from round impinging jets to a flat plate. Int. J. Heat Mass Transfer, 26, pp. 1857-1865.

Hrycak, P., D. T. Lee, J. W. Gauntner and J. N. B. Livingood, 1970. Experimental flow characteristics of a single turbulent jet impinging on a flat plate. NASA TN D-5690.

Huang, P. G., A. S. Majumdar and W. J. M. Douglas, 1984. Numerical prediction of fluid flow and heat transfer under a turbulent impingement jet with surface motion and crossflow. ASME paper 84-WA/HT-33.

Idelchik, I. E., 1994. Handbook of hydraulic resistance, 3rd ed. Boca Raton: CRC Press.

Kakac, S., and D. B. Spalding, 1979. Turbulent forced convection in channels and bundles. Washington: Hemisphere.

Kakac, S., R. K. Shah and A. E. Bergeles, 1983. Low Reynolds number flow heat exchangers. Washington:

Hemisphere.

Kasagi, N., and A. Matsunaga, 1995. Three-dimensional particle-tracking velocimetry measurement of turbulence statistics and energy budget in a backwards-facing step flow. Int. J. Heat Fluid Flow, 16, pp. 477-485.

Kasagi, N., and K. Nishio, 1991. Probing turbulence with three-dimensional particle-ttracking velocimetry. Exp. Ther. Fluid Sci., 4, pp. 601-612.

Katinas, V. I., R. V. Bakas, E. E. Perednis and V.A. Svedoscius, 1990. Effect of turbulence of the incident flow on flow-induced vibrations of tube bundles operating in crossflow. Fluid Mechanics-Soviet Research, 19, No. 5, pp. 9-17. 
Kawamura, H., 1979. Analysis of laminarization of heated turbulent gas using a two-equation model of turbulence. Proc., 2nd Intl. Symp. Turb. Shear Flow, London, pp. 18.16-18.21.

Kays, W. M., 1966. Convective heat and mass transfer. New York: McGraw-Hill.

Kays, W. M., and M. E. Crawford, 1980. Convective heat and mass transfer, 2nd Ed. New York: McGraw-Hill.

Kays, W. M., and A. L. London, 1955. Compact heat exchangers. Palo Alto: National Press.

Kent, J. C., N. Trigui, W.-C. Choi, Y. G. Guezennec and R.S. Brodkey, 1993. Photogrammetric calibrations for improved three-dimensional particle tracking velocimetry (3-D PTV). SPIE Proc. 2005, pp. 400-412.

Kim, K., B. Wiedner and C. Camci, 1995. Fluid dynamics and convective heat transfer in impinging jets through implementation of a high resolution liquid crystal technique. Part I: Flow and heat transfer experiments. Int. J. Turbo. and Jet Engines, 12, pp.

Kim, S., and G. C. Park, 1998. Analysis of turbulent mixing in rod bundles with an anisotropic turbulent diffusion model based on the flow pulsation phenomenon. Nuc. Tech., 122, pp. 284-294.

Knowles, K., and M. Myszko, 1995. Radial wall jets formed by high speed impinging jets. High Speed Jet Flows, ASME FED-Vol. 214, pp. 53-58.

Knowles, K., and M. Myszko, 1998. Turbulence measurements in radial wall jets. Exp. Thermal Fluid Sci., 17, pp. 7178.

Kohli, A., and K. A. Thole, 1997. A CFD investigation on the effects of entrance crossflow directions to film-coooling holes. Proc., Nat. Heat Transfer Conf., Baltimore.

Krauss, T., and L. Meyer, 1998. Experimental investigations of turbulent transport of momentum and energy in a heated rod bundle. Nuc. Eng. Design, 180, pp. 185-206.

Kreith, F., 1965. Reverse transition in radial source flow between two parallel plates. Phys. Fluids, $\underline{8}$, pp.1189-1190.

Kreith, F., E. Doughman and H. Kozlowski, 1963. Mass and heat transfer from an enclosed rotating disc with and

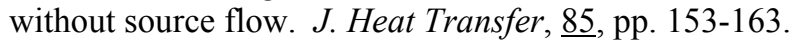

Landreth, C. C., and R. J. Adrian, 1990. Impingement of a low Reynolds number turbulent circular jet onto a flat plate at normal incidence. Exp. Fluids, $\underline{9}$, pp. 74-84.

Langhaar, H. L., 1942. Steady flow in the transition length of a straight tube. J. Appl. Mech., 9 , pp. A55-A58.

Laws, E. M., and J. L. Livesey, 1978. Flow through screens. Ann. Rev. Fluid Mech., 10, pp. 247-266.

Lee, J., and S.-J. Lee, 2000. The effect of nozzle aspect ratio on stagnation region heat transfer characteristics of elliptic impinging jet. Int. J. Heat Mass Transfer, $\underline{43}$, pp. 555-575.

Lee, X. C., C. F. Ma, Q. Zheng, Y. Zhuang and Y. Q. Tian, 1997. Numerical study of recovery effect and impingement heat transfer with submerged circular jets of large Prandtl number liquid. Int. J. Heat Mass Transfer, 40, pp. 2647-2653.

Leinhard V, J. H., R. S. Dahbura, H. F. Younis and C. H. Oh, 1996. Large area jet-array cooling modules for high heat fluxes. High Heat Flux Engineering III, SPIE Vol. 2855, pp. 66-81.

leWalle, J., D. E. Ashpis and K.-H. Sohn, 1997. Demonstration of wavelet techniques in the spectral analysis of bypass transition data. NASA TP-3555.

Lieber, B. B., A. P. Stancampiano and A. K. Wakhloo, 1997. Alteration of hemodynamics in aneurysm models by stenting: Influence of stent porosity. Annals Biomed. Eng., 25, pp. 460-469. 
Lindgren, E. R., 1953. Some aspects of the change between laminar and turbulent flow of liquids in cylindrical tubes. Arkiv Fysik, ㄱ, p. 293.

Liu, X., and J. H. Lienhard V, 1993. Extremely high heat fluxes beneath impinging liquid jets. J. Heat Transfer, 115, pp. $472-476$.

Lowe, M. L., P. H. Kutt, T. York, L. A. Kazanov and B. Leavy, 1993. Reconstruction of three-dimensional particle trajectories in flows through curved circular tubes. Exp. Fluids, 14, pp. 402-408.

Ma. C. F., Q. Zheng, S. C. Lee and T. Gomi, 1997. Impingement heat transfer and recovery effect with submerged jets of large Prandtl number liquid - I. Unconfined circular jets. Int. J. Heat Mass Transfer, 40, pp. 1481-1490.

Ma, C. F., Q. Zheng, H. Sun, K. Wu, T. Gomi and B. W. Webb, 1997. Local characteristics of impingement heat transfer with oblique round free-surface jets of large Prandtl number fluid. Int. J. Heat Mass Transfer, $\underline{40}$, pp. 22492259.

MacManus, D., J. Eaton, R. Barrett, J. Rickards and C. Swales, 1996. Mapping the flow field induced by a HLFC perforation using a high resolution LDV. AIAA paper 96-0097.

Marcroft, H. E., and M. V. Karwe, 1999. Flow field in a hot air jet impingement oven - Part I: A single impinging jet. J. Food Proc. and Preserv., $\underline{23}$, pp. 217-233.

Martin, H., 1977. Heat and mass transfer between impinging gas jets and solid surfaces. Adv. Heat Transfer, 13, pp. 160.

McCreery, G. E., 1999. Inner vortex formation in the flow visualization apparatus inlet plenum. Informal memorandum, INEEL, June.

McCreery, G. E., K. G. Condie, D. M. McEligot, J. C. Crepeau, R. Clarksean, Y. G. Guezennec and R. S. Brodkey, 1999. Flow visualization and velocity measurements in a model fuel storage canister. Proc., Global '99 - Int. Conf. on Future Energy Systems, Jackson, Wyo., August-September.

McCreery, G. E., M. G. McKellar and C. M. Stoots, 1993. Steady-state and transient moderator flow an ink dispersion tests. INEL technical report EGG-EE-11012.

McEligot, D. M., 1981. Uniform inlet conditions for the NPS subsonic cascade wind tunnel. Tech. rpt. NPS67-81019PR, U. S. Naval Postgraduate School, Monterey, Cal.

McEligot, D. M., and C. A. Bankston, 1969. Turbulent predictions for circular tube laminarization by heating. ASME paper 69-HT-52.

McEligot, D. M., C. W. Coon and H. C. Perkins, 1970. Relaminarization in tubes. Int. J. Heat Mass Transfer, $\underline{13}$, pp. 431-433.

McEligot, D. M., and H. Eckelmann, 1993. Turbulence structure in the viscous layer of converging flows. Proc., 9th Intl. Symp. Turb. Shear Flows, Kyoto, 1, pp. 8-5-1 to 8-5-6.

McEligot, D. M., and H. Eckelmann, 2001. Laterally converging flows: Part 3. Mean turbulence structure in the viscous layer. Manuscript in preparation.

McEligot, D. M., L. W. Ormand and H. C. Perkins, 1966. Internal low Reynolds number turbulent and transitional gas flow with heat transfer. J. Heat Transfer, $\underline{88}$, pp 239-245.

Mikic, B. B., Lj. B. Vujisic and J. Kapat, 1994. Turbulent transition and maintenance of turbulence; implication to heat transfer augmentation. Int. J. Heat Mass Transfer, 37, Suppl 1, pp. 425-431. 
Mikielewicz, D. P., 1994. Comparative studies of turbulence models under conditions of mixed convection with variable properties in heated vertical tubes. Ph.D. thesis, Univ. Manchester.

Moin, P. and K. Mahesh, 1998. Direct numerical simulation : A tool for research. Ann. Rev. Fluid Mech., 30, pp. 539578.

Moller, P. S., 1963. Radial flow without swirl between parallel discs. Aero. Quarterly, 14, pp. 163-186.

Möller, S. V., 1992. Single-phase turbulent mixing in rod bundles. Exp. Th. Fluid Sci., $\underline{5}$, pp. 26-33.

Morgan, P. G., and A. Saunders, 1960. An experimental investigation of inertia effects in viscous flow. Int. J. Mech. Sci., 2, pp. 8-12.

Morris, G. K., and S. V. Garimella, 1996. Orifice and impingement flow fields in confined jet impingement. Proc., Nat. Heat Transfer Conf., ASME HTD-Vol. 324, volume 2, pp. 101-106.

Morris, G. K., and S. V. Garimella, 1998. Orifice and impingement flow fields in confined jet impingement. $J$. Elec. Packaging, $\underline{120}$, pp. 68-72.

Morris, G. K., S. V. Garimella and J. A. Fitzgerald, 1998. Improved predictions of the flow field in submerged and confined impinging jets using the Reynolds stress model. Proc., 6th Intersociety Conference on Thermal and Thermomechanical Phenomena in Electronic Systems (ITherm'98), IEEE, Seattle, May, pp. 362-370.

Morris, G. K., S. V. Garimella and J. A. Fitzgerald, 1999. Flow-field prediction in submerged and confined impinging jets using the Reynolds stress model. J. Elec. Packaging, 121, pp. 255-262

Myszko, M., and K. Knowles, 1994. Development of a wall jet from an impinging, round, turbulent, compressible jet. AIAA paper 94-2327.

Nelson, E. S., 1987. Phase-averaged measurements of vortex interaction with a solid surface and the breakaway process. M.S. thesis, Illinois Inst. Tech.

Nishimura, M., S. Fujii, A. M. Shehata, T. Kunugi and D. M. McEligot, 2000. Prediction of forced gas flows in circular tubes at high heat fluxes. J. Nuclear Science and Technology (Atomic Energy Society of Japan), 37, pp. 581-594

Nishino, K., M. Samada, K. Kasuya and K. Torii, 1996. Turbulence statistics in the stagnation region of an axisymmetric impinging jet flow. Int. J. Heat Fluid Flow, 17, pp. 193-201.

Nishio, K., and N. Kasagi, 1989. Turbulence statistics measurement in a two-dimensional channel flow using a threedimensional particle tracking velocimeter. Proc., 7th Symp. Turb. Shear Flows, Stanford, pp. 22.1.1-22.1.6.

Nishio, K., N. Kasagi and M. Hirata, 1989. Three-dimensional particle tracking velocimetry based on automated digital image processing. J. Fluids Engr., 111, pp. 384-391.

Olsson, M., and L. Fuchs, 1998. Large eddy simulations of a forced semiconfined circular impinging jet. Phys. Fluids, 10, pp. $476-486$.

Orr, B., E. Thomson and R. S. Budwig, 1997. Drakeol 5 thermophysical property measurements. Technical report, Mech. Engr. Dept., U. Idaho.

Ouma, B. H., and S. Tavoularis, 1991. Flow measurements in rod bundle subchannels with varying rod-wall proximity. Nuc. Engr. Design, 131, pp. 193-208.

Pankhurst, R. C., and D. W. Holder, 1952. Wind-tunnel technique. London: Pittman. 
Papantoniou, D., and T. Dracos, 1990. Lagrangian statistics in open channel flow by 3-D particle tracking velocimetry. Engineering Turbulence Modelling and Measurements (Ed.: W. Rodi and E. N. Ganic), New York: Elsevier, pp. 315324.

Parker, J., and P. Merati, 1996. An investigation of turbulent Taylor-Couette flow using laser Doppler velocimetry in a refractive index matched facility. J. Fluids Engr., 118, pp. 810-818.

Parneix, S., M. Behnia and P. A. Durbin, 1999. Predictions of turbulent heat transfer in an axisymmetric jet impinging on a heated pedestal. J. Heat Transfer, $\underline{121}$, pp. 43-49.

Patel, V. C., and M.R. Head, 1969. Some observations on skin friction and velocity profiles in fully developed pipe and channel flows. Fluid Mech., $\underline{38}$, pp. 181-201.

Perkins, K. R., and D. M. McEligot, 1975. Mean temperature profiles in heated laminarizing air flows. J. Heat Transfer, 97 , pp. 589-593.

Perkins, K. R., K. W. Schrade and D. M. McEligot, 1973. Heated laminarizing gas flow in a square duct. Int. J. Heat Mass Transfer, $\underline{16}$, pp. 897-916.

Poskas, P. S., and V. J. Survila, 1983. Fluctuations of velocity of cross flow of air in the space between tubes in bundles. Heat Transfer-Soviet Research, 15, No. 1, pp. 75-86.

Prandtl, L., 1933. Attaining a steady airstream in wind tunnels. NACA TM 726.

Prithiviraj, M., and M. J. Andrews, 1998. Three-dimensional numerical simulation of shell-and-tube heat exchangers. Part I: Foundation and fluid mechanics. Num. Heat Transfer, Part A, $\underline{33}$, pp. 799-816.

Ramer, E. R., and F. D. Shaffer, 1992. Automated analysis of multiple-pulse particle image velocimetry data. Appl. Optics, $\underline{31}$, pp. 779-784.

Rehme, K., 1992. The structure of turbulence in rod bundles and the implications on natural mixing between the subchannels. Int. J. Heat Mass Transfer, $\underline{35}$, pp. 567-581.

Resnikoff, H. L., and R. O. Wells, 1998. Wavelet analysis. Berlin: Springer.

Reynolds, H. C., 1969. Internal low Reynolds number turbulent heat transfer. Ph.D. thesis, Univ. Arizona.

Reynolds, H. C., M. E. Davenport and D. M. McEligot, 1968. Velocity profiles and eddy diffusivities for fully developed turbulent, low Reynolds number pipe flow. ASME Paper 68-WA/FE-34 .

Robinson, O., and D. Rockwell, 1993. Construction of three-dimensional images of flow structure via particle tracking techniques. Exp. Fluids, 14, pp. 257-270.

Rohde, J. E., H. T. Richards and G. W. Metger, 1969. Discharge coefficients for thick plate orifices with approach flow perpendicular and inclined to the orifice axis. NASA TN D-5467.

Rotta, J. C., 1956. Experimentteller Beitrag zur Entstehung turbulenter Strömung in Rohr. Ingenieur-Archiev, 24, pp. 258 - .

Rowe, D.S., B. M. Johnson and J. G. Knudsen, 1974. Implications concerning rod bundle crossflow mixing based on measurements of turbulent flow structure. Int. J. Heat Mass Transfer, 17, pp. 407-419.

Rubel, A., 1980. Computations of jet impingement on a flat surface. AIAA J., $\underline{18}$, pp. 168-175.

San, J.-Y., C.-H. Huang and M.-H. Shu, 1997. Impingement cooling of a confined circular air jet. Int. J. Heat Mass Transfer, 스, pp. 1355-1364. 
Satake, S.-I., 1996. Personal communication, Kogakuin Univ., Tokyo.

Satake, S.-I., and T. Kunugi, 1998. Direct numerical simulation of an impinging jet into parallel disks. Int. J. Num. Meth. Heat and Fluid Flow, $\underline{8}$, pp. 768-780.

Satake, S.-I., T. Kunugi, A. M. Shehata and D. M. McEligot, 2000. Direct numerical simulation on laminarization of turbulent forced gas flows in circular tubes with strong heating. Int. J. Heat Fluid Flow, 21, pp. 526-534.

Schlichting, H., 1979. Boundary layer theory, 7th ed. New York: McGraw-Hill.

Scholten, J. W., and D. B. Murray, 1998. Heat transfer and velocity fluctuations in a staggered tube array. Int. J. Heat Fluid Flow, 19, pp. 233-244.

Schwartz, A. C., M. W. Plesniak and S. N. B. Murthy, 1999. Turbulent boundary layers subjected to multiple strains. J. Fluids Eng., 121, pp. 526-532.

Shehata, A. M., 1984. Mean turbulence structure in strongly heated air flows. Ph.D. thesis, Univ. Arizona.

Shehata, A. M., and D. M. McEligot, 1998. Mean turbulence structure in the viscous layer of strongly-heated internal gas flows. Part I: Measurements. Int. J. Heat Mass Transfer, 41, pp. 4297-4313.

Simon, T. W., S. W. Burd, C. J. Satterness, R. W. Kaszeta and R. Oke, 1999. Documenting complex flows in gas turbine gas path cooling systems. Proc., Colloquium on Turbomachinery - 1999 (Ed.: S. J. Song), Turbo and Power Machinery Research Center, Seoul National University, Korea, pp. 1-16.

Slayzak, S. J., R. Viskanta and F. J. Incropera, 1994. Effects of interactions between adjoining rows of circular, freesurface jets on local heat transfer from the impingement surface. J. Heat Transfer, 116, pp. 88-95.

Sparrell, J. K., 1995. Letter, Sparrell Engineering Research Corporation, P.O. Box 130, Damariscotta, Maine 04543, 14 March.

Smith, A. M. O., 1960. Remarks on transitions in a round tube. J. Fluid Mech., 7, pp. 565-576.

Stanescu, G., A. J. Fowler and A. Bejan, 1996. The optimal spacing of cylinders in free-stream cross-flow forced convection. Int. J. Heat Mass Transfer, $\underline{39}$, pp. 311-317.

Stoots, C. M., S. Becker, K. G. Condie, F. Durst and D. M. McEligot, 2001. A large-scale matched-index-ofrefraction flow facility for LDA studies of complex geometries. Exp. Fluids, 30, pp. 391-398.

Tan-Atichat, J., H. M. Nagib and R. I. Loerhke, 1982. Interaction of free-stream turbulence with screens and grids: a balance between turbulence scales. J. Fluid Mech., 114, pp. 501-528.

Tani, I., and Komatsu, 1966. Impingement of a round jet on a flat surface. Int. Congress Appl. Mech., Munich: Springer, pp. 672-676.

Taylor, G. I., 1944. Aerodynamic properties of gauze. Tech. rpt. ARC 7598, included as Appendix in ARC R\&M 2276, 1945.

Taylor, G. I., and G. K. Batchelor, 1949. The effect of wire gauze on small disturbances in a uniform stream. Quarterly J. Mech. Appl. Math., 2, Pt. 1, pp. 1-29.

Thompson, B. E., O. Bouchery and K. D. Lowney, 1995. Refractive-index-matching laser velocimetry for complex nozzle entrance flow. Laser Anemometry - 1995, ASME FED-Vol. 229, pp. 365-370.

Todreas, N. E., and M. S. Kazimi, 1990. Nuclear systems. New York: Hemisphere.

Tritton, D. J., 1977. Physical fluid dynamics. New York: Van Nostrand Reinhold. 
Tropea, C., 1995. Laser Doppler anemomentry: recent developments and future challenges. Meas. Sci. Tech., $\underline{6}$, pp. 605-619.

Tufillaro, N. B., T. Abbott and J. Reilly, 1992. An experimental approach to nonlinear dynamics and chaos. Redwood City, Ca.: Addison-Wesley.

Ueda, T., H. Imaizumi, M. Mizimoto and I. G. Sheppard, 1997. Velocity statistics along the stagnation line of an axisymmetric stagnating turbulent flow. Exp. Fluids, 22, pp. 473-481.

Ushijima, S., and N. Tanaka, 1996. Three-dimensional particle tracking velocimetry with laser-light sheet scannings. $J$. Fluids Engr., 118, pp. 352-357.

Velichko, V. I., V. A. Pronin and N. Yassin, 1993. Heat transfer, hydrodynamics and energy efficiency in closely packed tube bundles in cross flow. Heat Transfer Research, 25, pp. 629-633.

Watterson, J. K., W. N. Dawes, A. M. Savill and A. J. White, 1999. Predicting turbulent flow in a staggered tube bundle. Int. J. Heat Fluid Flow, 20, pp. 581-591.

Watmuff, J. H., 1997. Detrimental effects of almost immeasurably small free-stream nonuniformities generated by wind tunnel screens. AIAA paper 97-0228.

Webb, B. W., and C.-F. Ma, 1995. Single phase liquid jet impingement heat transfer. Adv. Heat Transfer, 26, pp. 105217.

Wilfert, G., and S. Wolff, 1999. Influence of internal flow on film cooling effectiveness. ASME paper 99-GT-258, Int. Gas Turbine Conf., Indianapolis.

Wilson, A. S., and M. K. Bassiouny, 2000. Modeling of heat transfer for flow across tube banks. Chem. Eng. Processing, $\underline{39}$, pp. 1-14.

Wolfshtein, M., 1970. Some solutions of the plane turbulent impinging jet. ASME paper 70-FE-27.

Womac, D. J., F. P. Incropera and S. Ramadhyani, 1994. Correlating equations for impingment cooling of small heat sources with multiple circular liquid jets. J. Heat Transfer, 116, pp. 482-486.

Yanta, W. J., and R. A. Smith, 1973. Measurements of turbulence-transport properties with a laser Doppler velocimeter. AIAA paper 73-169.

Yapici, S., S. Kuslu, C. Ozmetin, H. Ersahan and T. Pekdemir, 1999. Surface shear stress for a submerged jet impingement using electrochemical technique. J. Appl. Electrochem., 29, pp. 185-190.

Zhao, Y., and R. S. Brodkey, 1998. Averaged and time-resolved, full-field (three-dimensional) measurements of unsteady opposed jets. Canadian J. Chem. Engr., $\underline{76}$, pp. 536-545.

Zukauskas, A., 1972. Heat transfer from tubes in cross flow. Adv. Heat Transfer, $\underline{8}$, pp. 93-160. 
